\title{
Stockpile Stewardship Program Strategy
}

\author{
W. Shotts
}

December 1, 1994

U.S. Department of Energy

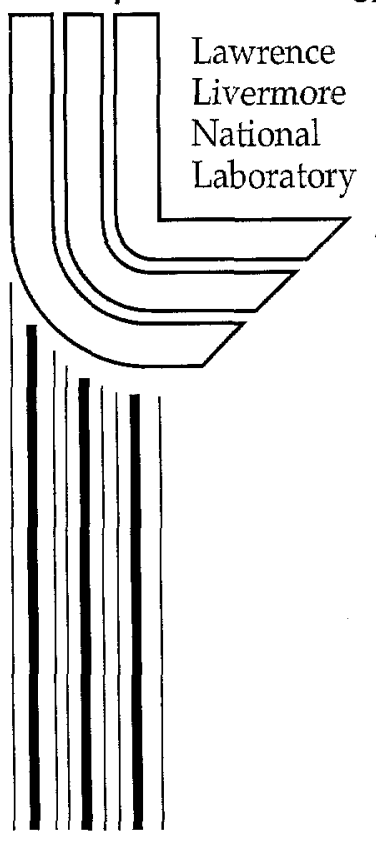




\section{Stockpile Stewardship Program Strategy}

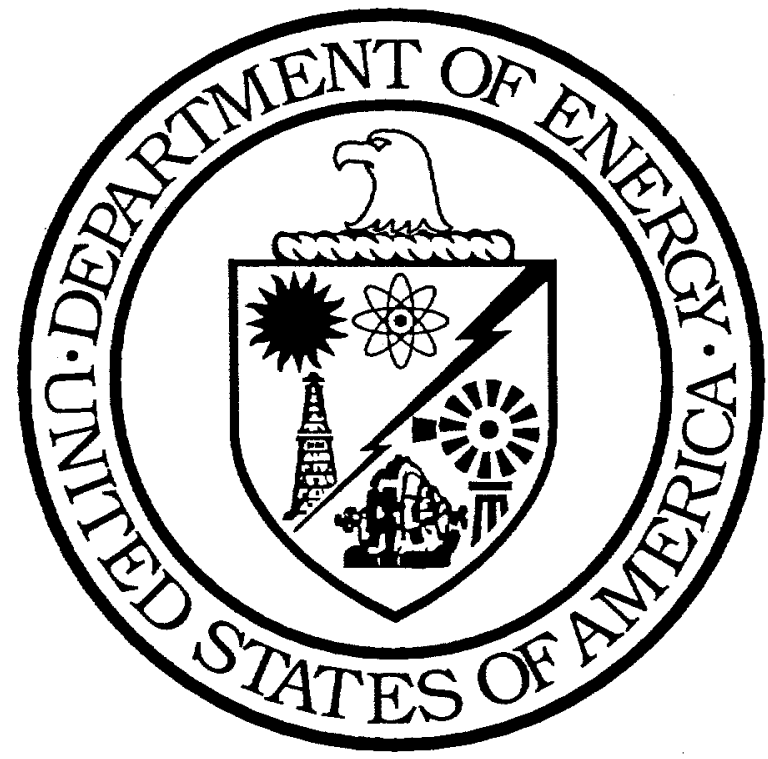

December 1994 


\section{DISCLAIMER}

This document was prepared as an account of work sponsored by an agency of the United States Government. Neither the United States Government nor the University of California nor any of their employees, makes any warranty, express or implied, or assumes any legal liability or responsibility for the accuracy, completeness, or usefulness of any information, apparatus, product, or process disclosed, or represents that its use would not infringe privately owned rights. Reference herein to any specific commercial products, process, or service by trade name, trademark, manufacturer, or otherwise, does not necessarily constitute or imply its endorsement, recommendation, or favoring by the United States Government or the University of California. The views and opinions of authors expressed herein do not necessarily state or reflect those of the United States Government or the University of California, and shall not be used for advertising or product endorsement purposes.

This report has been reproduced directly from the best available copy.

Available to DOE and DOE contractors from the Office of Scientific and Technical Information P.O. Box 62, Oak Ridge, TN 37831

Prices available from (615) 576-8401, FTS 626-8401

Available to the public from the National Technical Information Service

US. Department of Commerce 5285 Port Royal Rd., Springfield, VA 22161

Work performed under the auspices of the U.S. Department of Energy by Lawrence Livermore National Laboratory under Contract W-7405-Eng-48. 


\title{
Stockpile Stewardship Program Strategy
}

\author{
December 1994
}




\section{Contents}

Preface

Executive Summary.......................................................................................................................................1

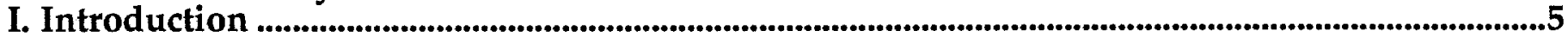

The Goal of Stockpile Stewardship.........................................................................................................

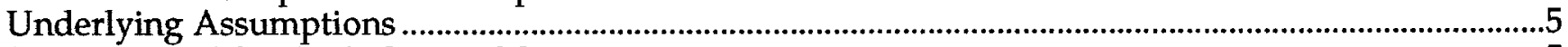

Science-Based Stockpile Stewardship ............................................................................................................

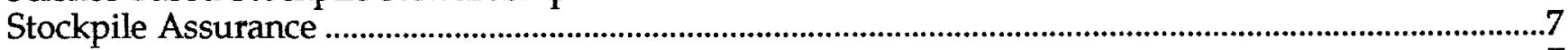

Constraints on Nuclear Weapons............................................................................................................

Stewardship Responsibilities ......................................................................................................................

Maintaining Confidence in the Enduring Stockpile ...........................................................................

Hedging Against the Uncertain Future .....................................................................................................11

Preserving a Unique Knowledge Base.......................................................................................................11

Partnership with U.S. Industry...................................................................................................................13

Roadmap for the Report .......................................................................................................................14

Recommendations for the U.S. Stockpile Stewardship Program ...............................................................14

Nuclear Components: Primaries ..........................................................................................................14

Nuclear Components: Secondaries ................................................................................................14

Nonnuclear Components.......................................................................................................................14

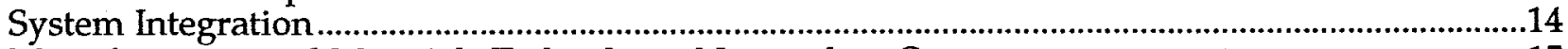

Manufacturing and Materials Technology: Nonnuclear Components.............................................15

Manufacturing and Materials Technology: Nuclear Components....................................................15

Stockpile Surveillance ...................................................................................................................................15

Computational Simulations..................................................................................................................15

The Nevada Test Site and Test Readiness ............................................................................................15

Technology Partnerships with U.S. Industry........................................................................................15

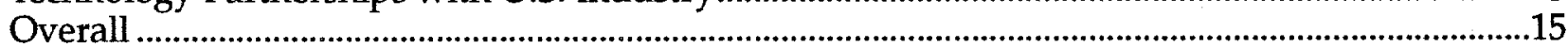

Planning in a Rapidly Changing Environment..........................................................................................16

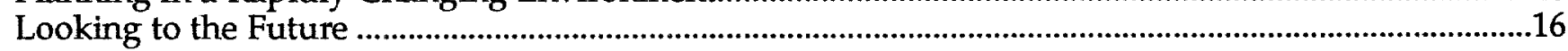

II. Introduction to Nuclear Weapons and Weapon Physics .....................................................................17

Operation of a Typical Nuclear Weapon.........................................................................................................17

A Science-Based Approach to the Physics of a Nuclear Weapon ..................................................................17

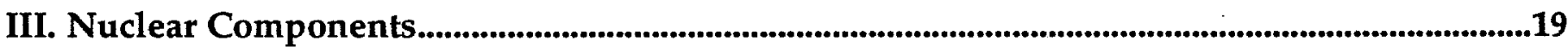

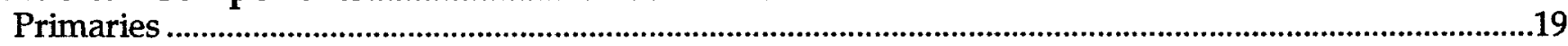

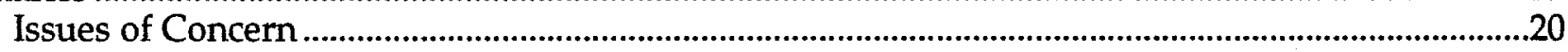

High-Explosive Detonation and Burn ...................................................................................2

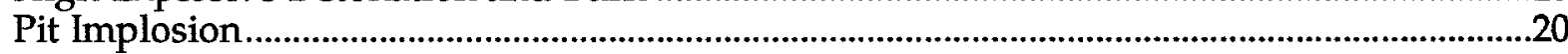

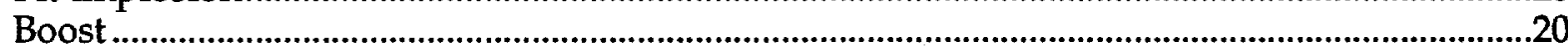

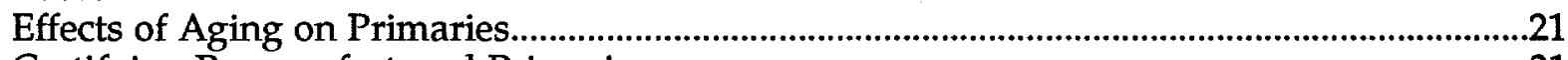

Certifying Remanufactured Primaries..............................................................................................21

Improvements to Meet Changes in Requirements........................................................................22

The Program for Addressing these Issues...........................................................................................23

Enhanced Predictive Capability for Primaries ……………………………………………….....23

New Experimental Measurements ..............................................................................................23

Hydronuclear Experiments and Hydrodynamic Tests....................................................................23

Near-Term Improvements in Hydrodynamic Testing .....................................................................24

Improved Understanding of Materials Properties........................................................................2 


\section{Contents}

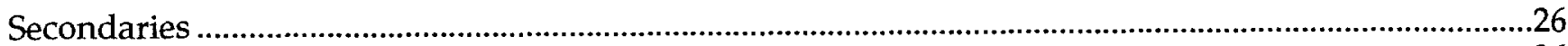

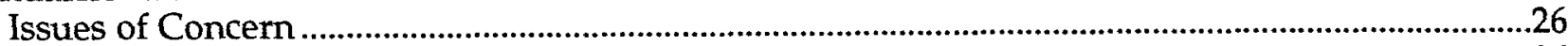

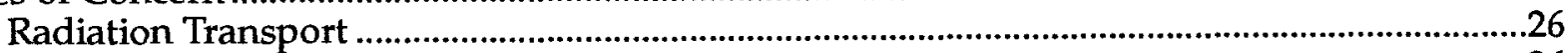

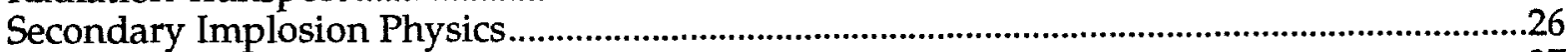

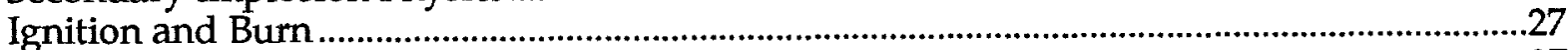

Weapon Output and Effects.........................................................................................................27

Effects of Aging on Secondaries and Stewardship of the Aging Stockpile..............................27

Certifying Remanufactured Secondaries .................................................................................28

The Program for Addressing these Issues .......................................................................................28

Improved Predictive Capability for Secondaries ...................................................................28

Improved Experimental Capabilities ..........................................................................................29

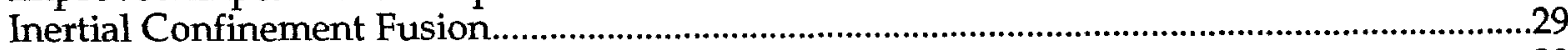

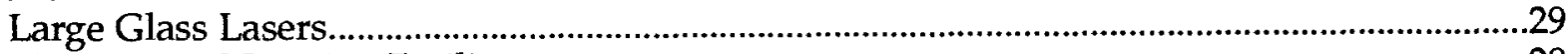

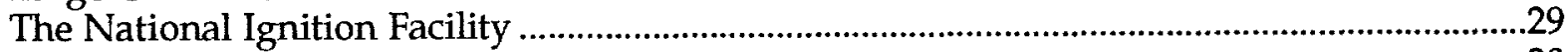

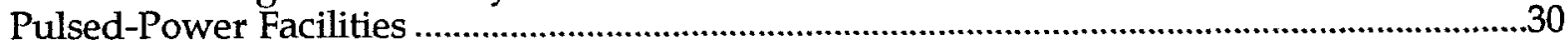

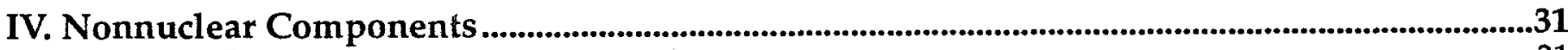

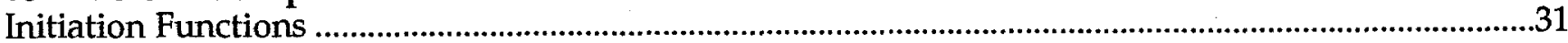

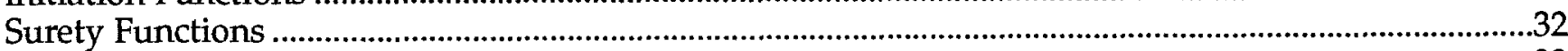

Electrical Nuclear Detonation Safety …........................................................................................33

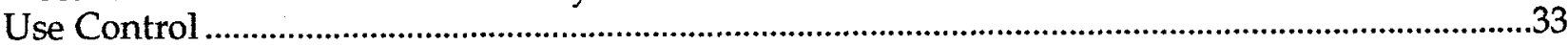

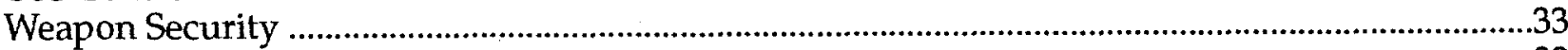

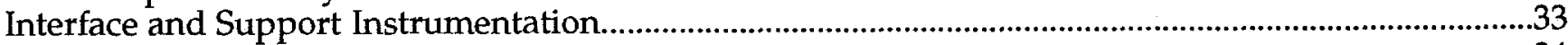

A Science-Based Approach to Weapons Engineering ..................................................................................34

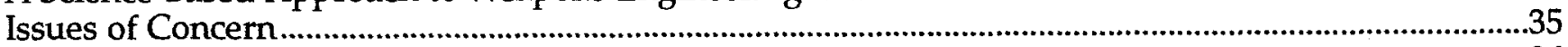

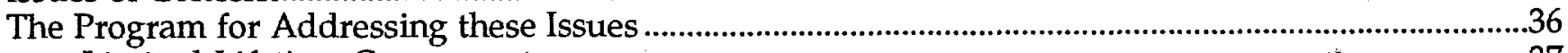

Limited-Lifetime Components.............................................................................................................37

Enhanced Nuclear Detonation Safety .....................................................................................................37

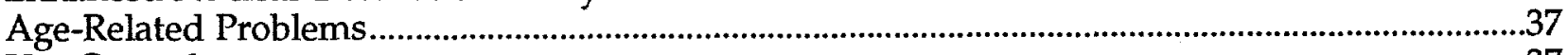

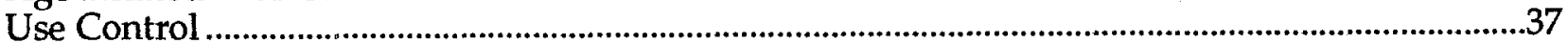

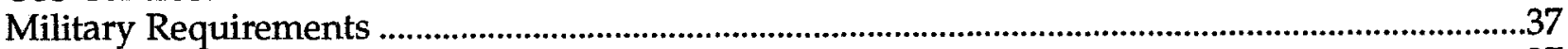

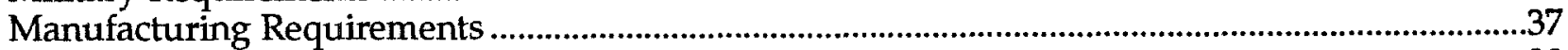

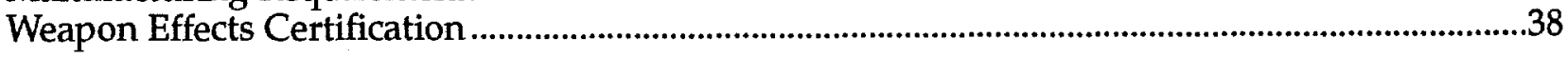

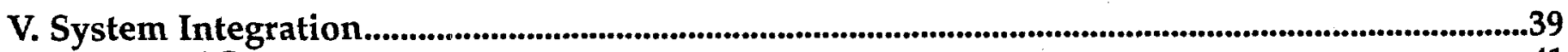

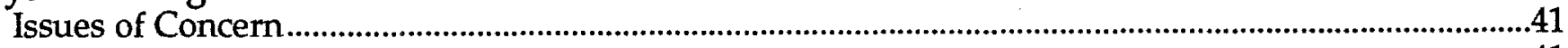

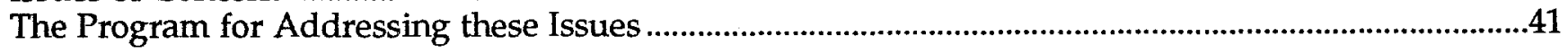

VI. Manufacturing and Materials Technology

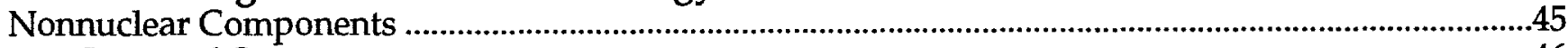

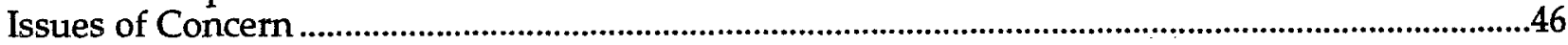

The Program for Addressing these Issues ............................................................................................46

Concurrent Engineering and Agile Manufacturing.....................................................................47

Flexible Workforce...............................................................................................................................47

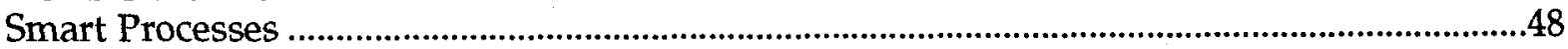

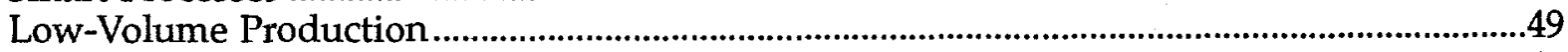

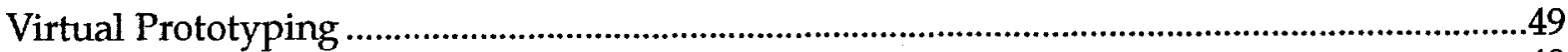

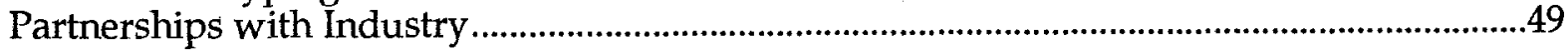




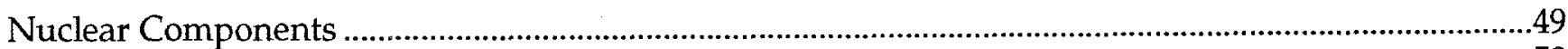

Key Issues and the Program for Addressing Them .........................................................................50

Issues in Remanufacturing and Recertification......................................................................51

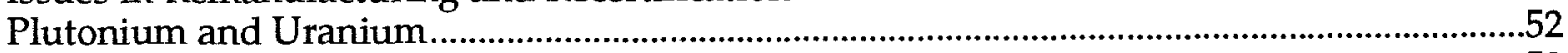

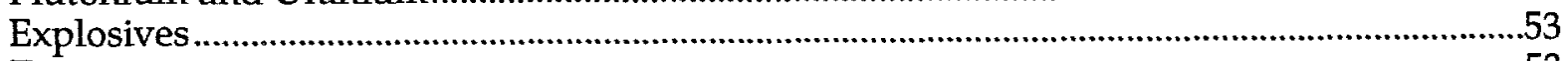

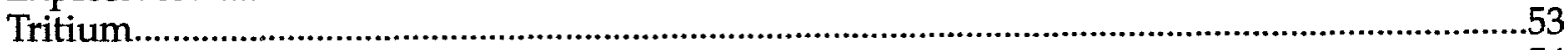

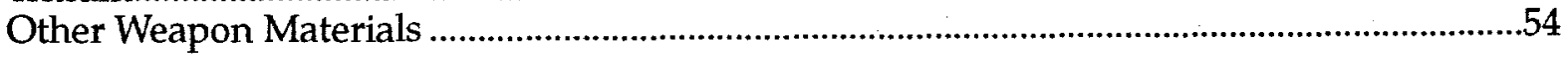

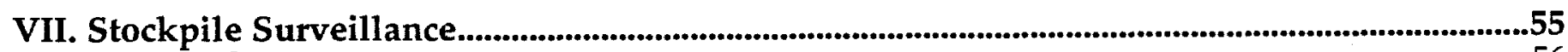

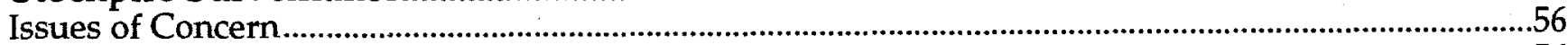

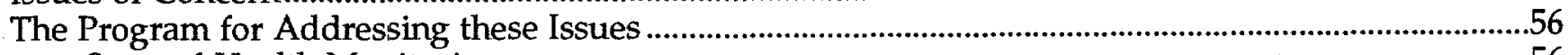

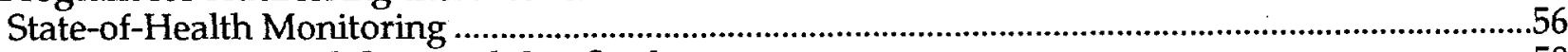

Materials Aging and Compatibility Studies .................................................................................58

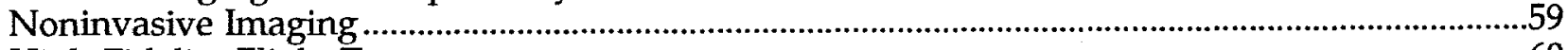

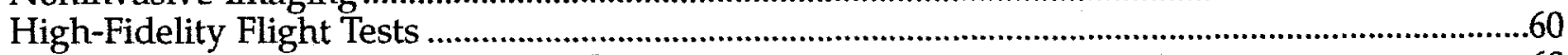

Hydrodynamic Tests and Hydronuclear Experiments ................................................................6

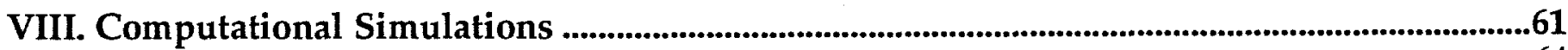

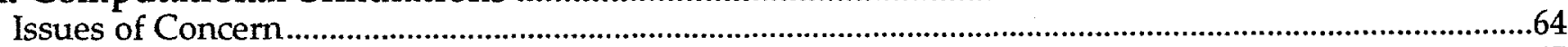

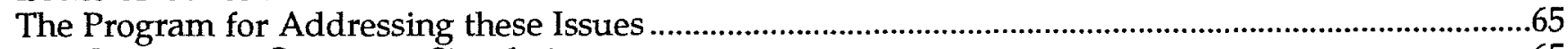

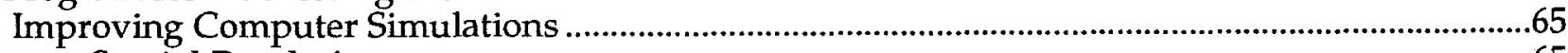

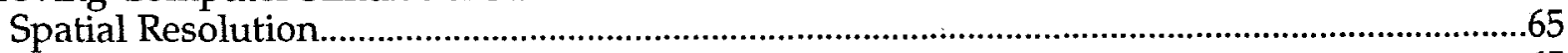

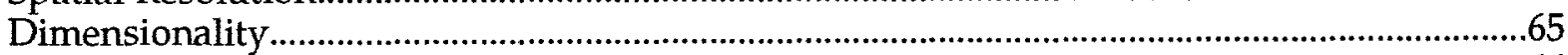

Physical Processes ......................................................................................................................66

Hardware, System Software, and Application Software ............................................................67

Increasing Computational Speed ...........................................................................................67

Massively Parallel Processing ....................................................................................................67

Moving Simulation Codes to MPP ............................................................................................67

Expanding Computer Networks and Storage Systems........................................................67

Improving Computational Tools................................................................................................68

The Computational Environment of the Future ..............................................................................68

IX. The Nevada Test Site and Test Readiness .........................................................................................71

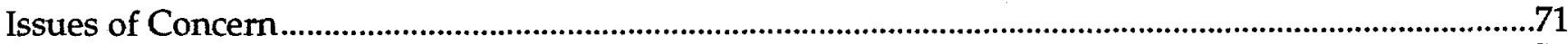

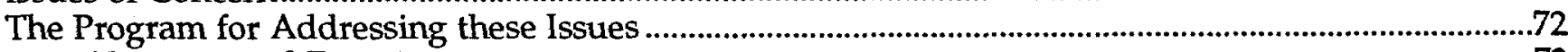

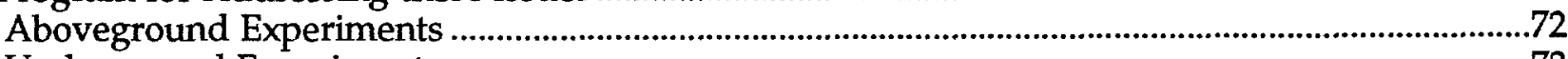

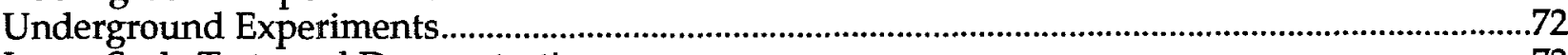

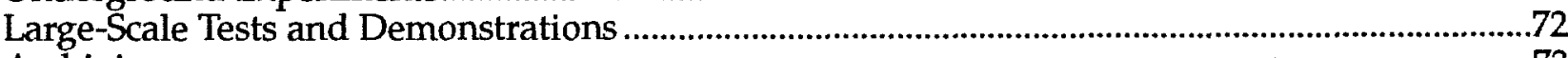

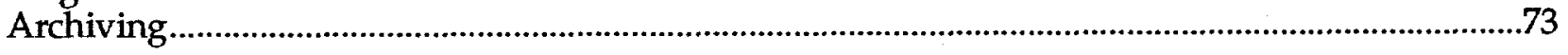

X. Technology Partnerships with U.S. Industry .......................................................................................75

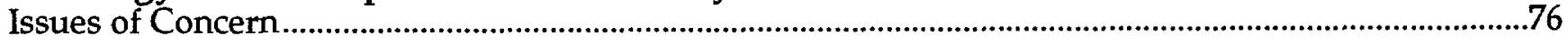

XI. Stockpile Stewardship and Other National Needs .................................................................77

Industrial Partnerships ..........................................................................................................................77

Counterproliferation and Nonproliferation ...........................................................................................77

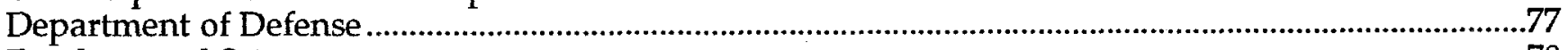

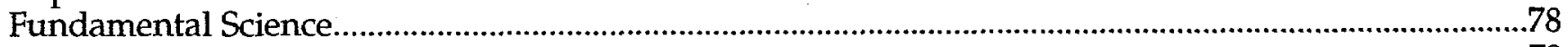

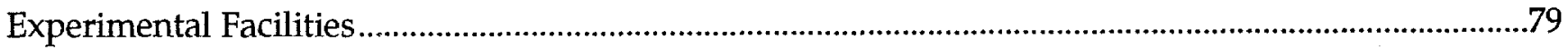




\section{Contents}

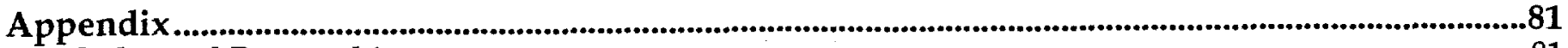

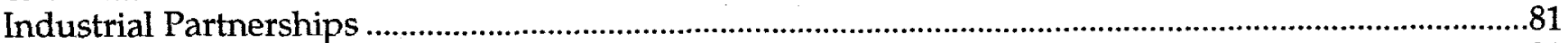

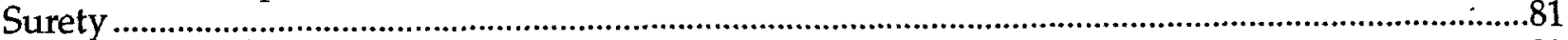

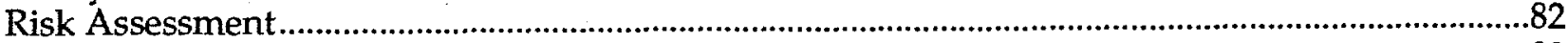

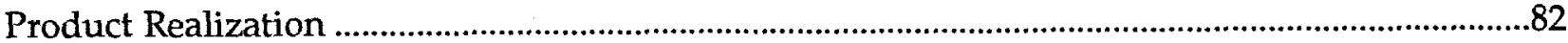

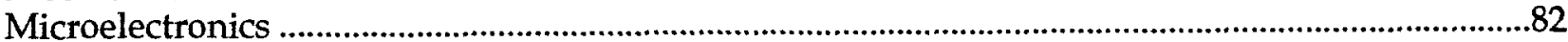

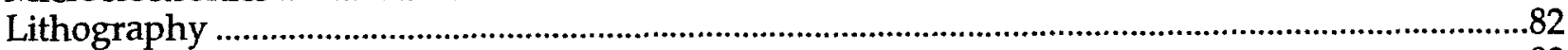

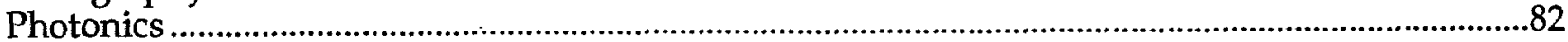

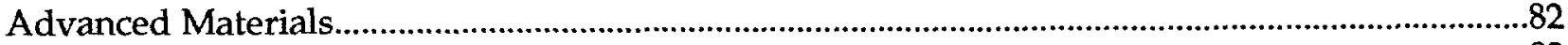

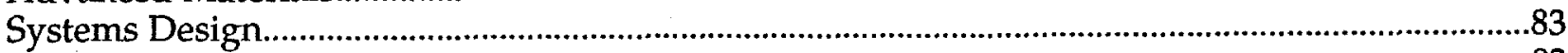

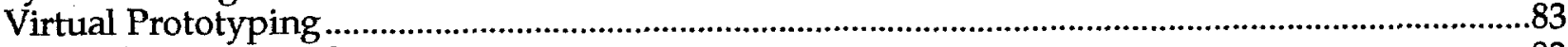

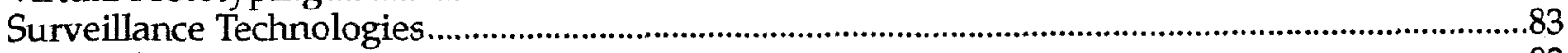

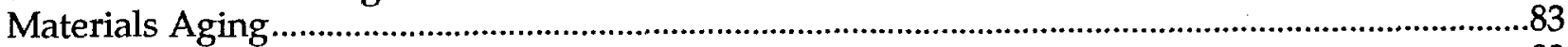

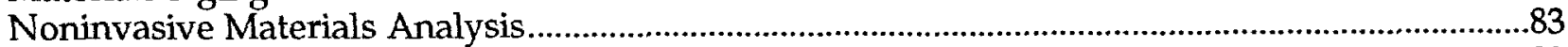

Counterproliferation and Nonproliferation .....................................................................................83

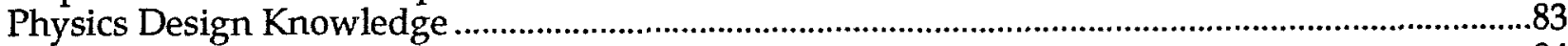

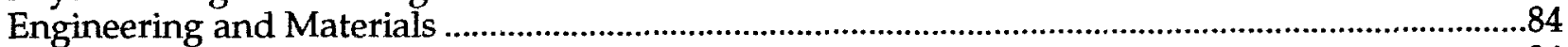

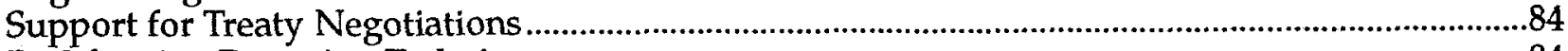

Proliferation Detection Techniques ...................................................................................................84

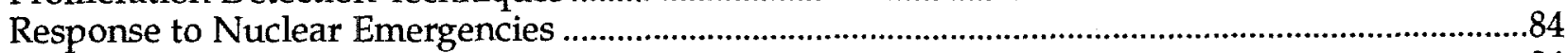

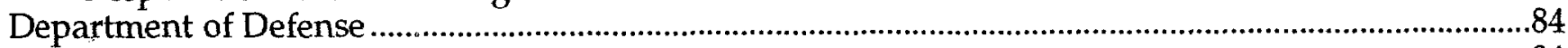

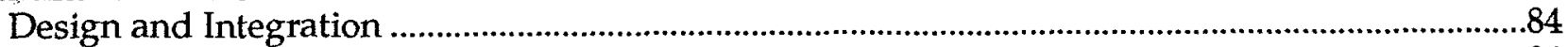

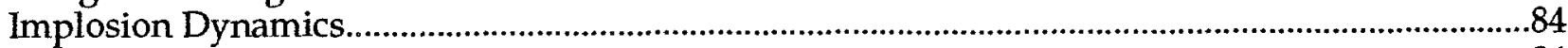

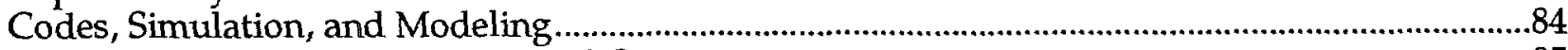

Diagnostics, Data Management, and Communications....................................................................85

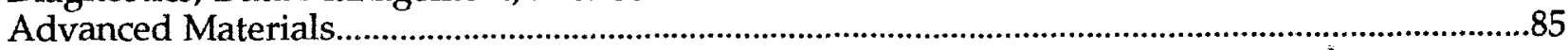

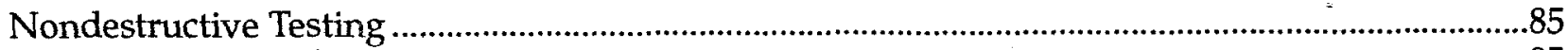

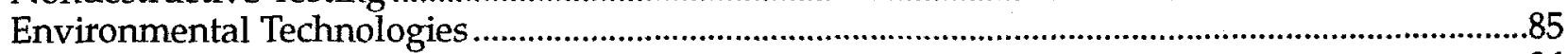

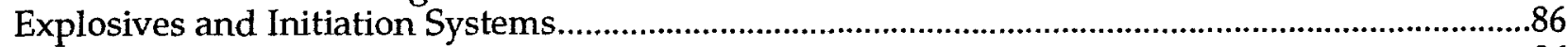

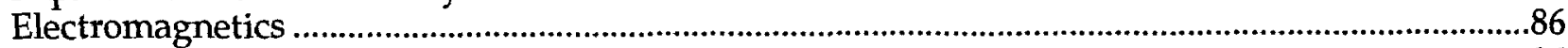

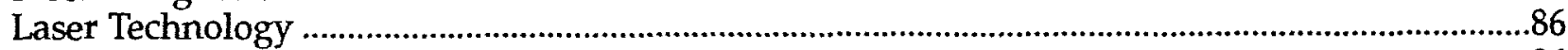

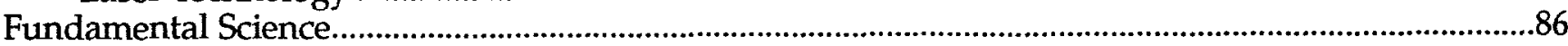

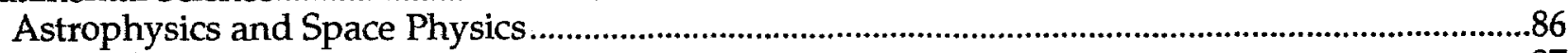

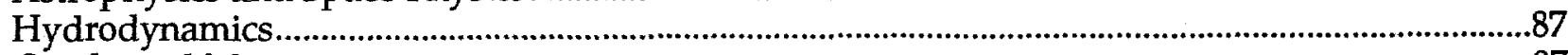

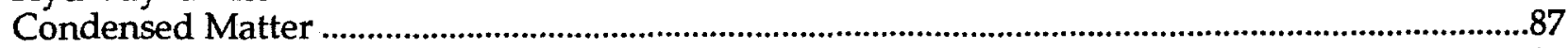

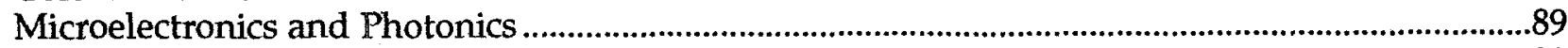

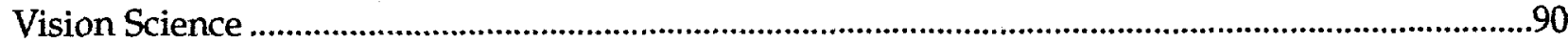

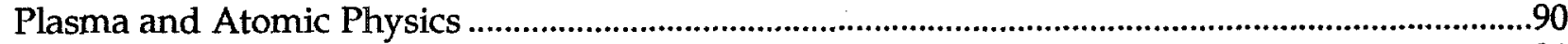

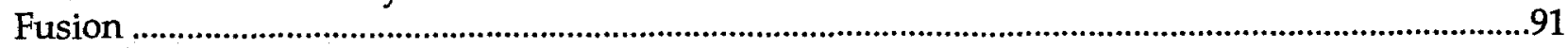

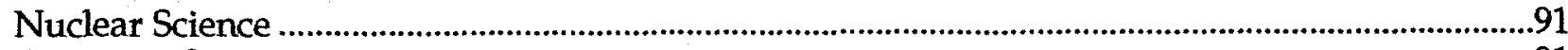

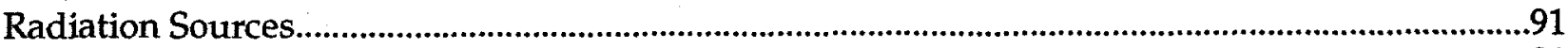

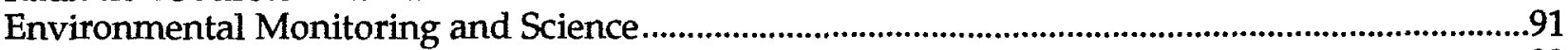

Weapons Systems Tests and Demonstrations....................................................................................92

High Explosives and Nuclear Materials Tests .....................................................................................92

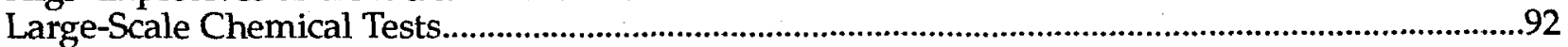

Conventional Numitions Tests and Demonstrations …........................................................................92

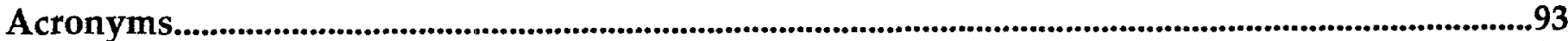

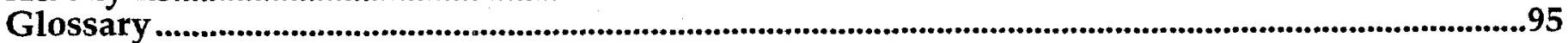




\section{Preface}

I $\mathrm{n}$ announcing the extension of the moratorium on nuclear testing, President Clinton reaffirmed the importance of maintaining confidence in the enduring U.S. nuclear stockpile: "To assure that our nuclear deterrent remains unquestioned under a test ban, we will explore other means of maintaining our confidence in the safety, reliability, and performance of our weapons." To this end, the Department of Energy's nuclear weapon program is undergoing fundamental changefrom advancing military characteristics to maintaining the reliability and safety of the existing stockpile. Consequently, a new strategy was needed to meet these new mission requirements. This report summarizes the new strategy.

The fundamental premise of the new strategy lies in the ability to respond to problems in monitoring and maintaining the existing stock- pile by preserving specialized facilities, maintaining the skill and knowledge bases, and advancing our understanding of nuclear weapon physics necessary to manage the nuclear future in an era without nuclear testing. The foundation of this Sciencebased Stockpile Stewardship strategy was laid at a workshop attended by DOE officials, DoD customers, stakeholders from other government agencies, nuclear weapon experts, and members of the scientific community. The principles of the evolving strategy were regularly discussed during the formative stages with the primary customers and stakeholders and reviewed by the JASONs. This report summarizes the strategy as it now exists, but we recognize that stockpile stewardship must be a continuing process - updated as necessary to respond to national security objectives.

Victor H. Reis

Assistant Secretary for Defense Programs

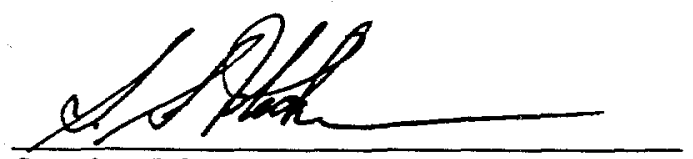

Siegfried S. Hecker, Director

Los Alamos National Laboratory

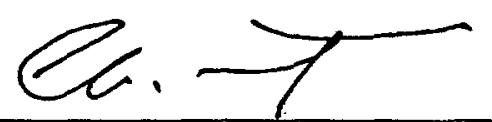

Albert Narath, President Sandia National Laboratories

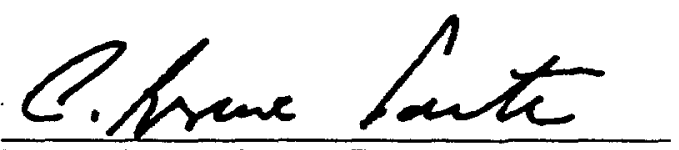

Bruce Tarter, Acting Director Lawrence Livermore National Laboratory 


\section{Executive Summary}

$\mathbf{T}$ he Cold War, the major driving force behind the pace and size of the U.S. nuclear weapons program, has ended with the dissolution of the Soviet Union, and major geopolitical changes are continuing to occur. As a result, the nation's nuclear weapons program has changed dramatically, and additional changes are occurring in response to such factors as: - The reduction of the nuclear threat achieved with the signing of the START I (1991) and START II (1993) agreements. - The unilateral halt to the development and production of new nuclear weapon systems by the U.S. in 1992 and, at the same time, the reduction in the number of U.S. nuclear weapons on alert. - The moratorium on underground nuclear testing, which was extended by President Clinton in July 1993 and again in March 1994. - The desire of the U.S. to negotiate a Comprehensive Test Ban Treaty and to encourage the broadest possible participation in the Nuclear Non-Proliferation Treaty.

- The closure of elements of the U.S. nuclear weapons production complex no longer needed for the smaller, less diverse enduring stockpile.

- Tighter constraints on environmental impact and workplace safety.

In addition, the Nuclear Posture Review, led by the Department of Defense, has addressed possible changes in U.S. nuclear policy (e.g., deployment status, targeting, force structure). The resulting recommendations and decisions dictate little change in the future U.S. nuclear weapons stockpile.

In announcing the extension of the moratorium on nuclear testing, President Clinton reaffirmed the importance of maintaining confidence in the enduring U.S. nuclear stockpile:

"To assure that our nuclear deterrent remains unquestioned under a test ban, we will explore other means of maintaining our confidence in the safety, reliability, and the performance of our own weapons."

Developing these "other means" will require breakthroughs in unexplored areas of science and technology as well as a fundamental change in the way confidence in the stockpile is maintained. The challenge of stockpile confidence is exacerbated by other constraints, including system development and production inactivity, facility closures, fiscal realities, and environmental and safety issues. The path to meeting this challenge is strewn with uncertainties-in particular, uncertainty as to the time it will take to make the required scientific and technological breakthroughs and uncertainty regarding how much or how quickly confidence in the existing stockpile might decline. Nonetheless, the DOE is committed to developing, as quickly as possible, the necessary means for maintaining stockpile confidence.

The Stockpile Stewardship Program is one of two elements (the other being the Stockpile Management Program) of the Department of Energy initiative to respond to this challenge in an effective and cost-efficient manner. These two integrated, interdependent programs comprise much of the Department of Energy's contribution to the nation's nuclear deterrent.

Two major changes are occurring in the nuclear weapons program. First is a move from nuclear-test-based certification of nuclear weapon reliability and safety and from replacement-based assurance of weapon performance to certification and assurance based on thorough scientific understanding and predictive models of performance-that is, sciencebased stockpile stewardship. Second is a move from deterrence based on the capacity to build large numbers of nuclear weapons to deterrence based on the capability to respond to any and all reliability and safety problems that may affect the existing stockpile - that is, capability-based deterrence. This capability includes the ability to replace weapons after they reach their useful lifetime and the ability to upgrade systems to improve their safety, reliability, or lifetime cost. In keeping with national nonproliferation goals, it does not include specific provisions for providing enhanced performance.

The three national weapons laboratories-Los Alamos, Livermore, and Sandia-work together to provide the nation with the technical basis for a safe, reliable, and credible nuclear deterrent. The program strategy described in this report 
provides the technical roadmap for the U.S. science-based Stockpile Stewardship Program. The fundamental objectives of this strategy are to maintain the safety and reliability of the existing stockpile and to meet all new requirements by the military services for system modifications, repairs, and replacements. To fulfill this responsibility, we must maintain the appropriate research foundations, critical skills, and judgment bases. We must also initiate new or accelerated efforts to mitigate the various new constraints on the program.

The following six recommendations are crucial to this program.

1. New experimental facilities and capabilities are needed to mitigate, to the extent possible, the unavailability of new experimental data from nuclear tests. These new facilities and capabilities will enable us to gain an improved understanding of the underlying physics and phenomena of nuclear weapons, to acquire and benchmark new data to existing databases, and to test and validate the computer codes that will provide the future basis for ensuring safety, reliability, and performance. With these new facilities and experimental capabilities, we will also be able to make important contributions to fundamental and applied science and to develop technologies that will also benefit private industry and the civilian sector. The facilities and experimental capabilities of highest priority are:
- The National Ignition Facility. This facility would provide valuable data, such as material opacities and equations of state, for predicting and assessing the performance of nuclear assemblies. The National Ignition Facility would also provide an extraordinary capability to test the complex numerical codes used in weapons test calculations. In addition, this facility is a critical step in the international effort to evaluate the feasibility of inertial confinement fusion for energy production; it would also be used to study important areas of fundamental science.

- Advanced Hydrodynamic Test Capabilities. New hydrodynamic test capabilities are required to address issues of weapon safety and aging and to provide an experimental validation of our physics understanding and predictive capabilities for primary stages. In particular, we need improved dynamic radiography with greater spatial resolution, time sequences, and three-dimensional imaging. Most important are completion of the Dual-Axis Radiographic Hydro Test (DARHT) facility (at Los Alamos) and an improved gamma-ray detector. In the longer term, an improved radiographic facility that provides for imaging on more than two axes and multiple time frames may also be required.

Hydronuclear experiments would have nuclear yields equivalent to a few pounds or less of high explosive. If authorized by the President, these experiments would provide data that cannot be obtained by any other means to assess nuclear weapon safety and age-related concerns. These experiments would also provide the capability to benchmark current primary performance so that we can assess, at a future date, changes in performance due to aging. The stockpile stewardship strategy described in this report allows for hydronuclear experiments (if authorized by the President) by maintaining the nuclear testing infrastructure but does not specify particular experiments.

- Pulsed-Power Facilities. Facilities that generate pulsed power to form high-energydensity and high-power-density materials systems can be used to gather important data for understanding the physics of primary and secondary stages and to test and validate the computer codes for assessing nuclear weapon safety. The proposed Atlas facility (at Los Alamos) would permit new laboratory studies of hydrodynamic physics on scale sizes of a few centimeters and at temperatures high enough to bring significant ionization processes into play. This is an important regime for understanding the performance of primaries and secondaries and for testing our predictive computer codes. Further in the future, a new fast pulsed-power facility, such as the Jupiter facility planned by Sandia, may be required to provide data at higher temperatures than can be reached with Atlas. Data at temperatures higher than are possible with Atlas 
might be necessary to critically test some key computer simulations. If built, Jupiter would also provide an improved capability for certifying the tolerance of weapon components to $x$ rays.

2. Substantial advances in computational capabilities are required for science-based stockpile stewardship. Without nuclear testing, numerical simulation and computer modeling in conjunction with expert judgment will be the principal means of assessing the safety and performance of nuclear assemblies and weapons. Thousand-fold increases in computer speed and data storage are needed to permit the incorporation of more complete physics, modeling in three dimensions, and other improvements for completeness and accuracy. We must also improve our computational capabilities for assessing electrical nuclear detonation safety, for system- and sub-

system-level modeling, and for model-based low-volume manufacturing of affordable weapon components.

\section{New stockpile surveillance} capabilities will be key to ensuring confidence in the enduring U.S. stockpile at an affordable cost. The aging, smaller, and less diverse stockpile raises new issues that require new approaches. In particular, we must develop ways to prevent common-mode failures that could rapidly compromise a substantial portion of the stockpile. We must also develop more sensitive methods to assess the degradation in safety or reliability that may occur as weapons age beyond their design lifetime. In addition, we must develop nondestructive testing techniques so we can examine and assess the condition of weapons and weapon components. The highest priority initiatives for stockpile surveillance are:

- Noninvasive imaging. Higher resolution radiographs of nuclear components or weapons using $x$ rays or neutrons would allow us to detect internal defects without disassembling or sacrificing the test sample. This would make it possible to inspect a nuclear system and then, if everything is satisfactory, return it to the stockpile. X-ray and neutron imaging are complementary techniques; $x$ rays can be used for imaging high-atomic-weight materials and neutrons for lowatomic-weight materials. Substantial improvements over current capabilities are needed, particularly in spatial resolution, to meet stockpile stewardship requirements. Completion and operation of the Los Alamos Neutron Scattering Center (LANSCE) upgrade will provide a required neutron imaging capability as well as a capability for a wide range of materials science experiments.

- Real-Time Weapon SelfDiagnostics. Integrated self-test features (microsensors and miniature measuring devices with built-in intelligence capabilities) could reduce stockpile maintenance costs while providing the unprecedented ability to detect (and even predict) agerelated defects that may degrade weapon safety or performance.
This technology would also find broad use in commercial products and the private sector, making it a prime candidate for dual-use/ dual-benefit application.

4. New capabilities for materials and manufacturing, both nuclear- and nonnuclear-related, are required. Of particular importance are new technologies for nuclear materials that are both less costly to maintain and operate and environmentally benign. Research for less costly ways to produce tritium is essential. The production of nonnuclear components must use technologies that are appropriate for rapid prototyping of highquality products in small lots at affordable costs. Priority areas include model-based concurrent design of products and manufacturing processes. Technologies for virtual prototyping (that is, complete simulation of the product design, performance, and manufacturing processes before any hardware is produced) will be developed for appropriate applications.

5. The systems engineering infrastructure and the capability to design, engineer, and certify nuclear weapons must be preserved.

Assessing and certifying the safety of nuclear weapons are complex tasks and require a unique knowledge base. We must develop system-level models that can be used by future system engineers to assess weapon safety, reduce the time to develop replacement components (as needed to correct safety or performance problems), reduce the reliance on physical testing to 
ensure safety and performance, and ensure the safe dismantlement of the weapons in the future. In order to preserve the technical know-how and knowledge base unique to nuclear weapons, we need to maintain active prototype system-development programs that are both technically challenging and useful (e.g., for enhanced safety).

6. The capability to resume nuclear testing must be maintained, as mandated by the President. The stockpile stewardship strategy allows for this by maintaining a necessary infrastructure at the Nevada Test Site and by preserving the experimental expertise at the weapons laboratories.
In summary, maintaining confidence in the U.S. nuclear stockpile while honoring the moratorium on nuclear testing is a significant technical challenge. Meeting this challenge will require a substantial investment in new facilities and enhanced capabilities. It will also require the expert judgment of outstanding scientists and engineers.

Like all forward-looking research, the road to meeting these objectives cannot be defined with certainty at the outset. There will undoubtedly be some setbacks and changes in direction along the way. The strategy presented here is a starting point and will evolve as new understanding and new techniques are acquired.

Because this stockpile stewardship strategy calls for breakthroughs in unexplored areas of science and technology, the time to reach the intended goals is uncertain. Given the uncertainties of how quickly the confidence in the stockpile will decline and how long it will take to develop appropriate ways to bring stockpile confidence back to an acceptable level, it is prudent if not essential that we embark on the required scientific, technical, and programmatic thrusts as quickly and as aggressively as possible. 


\section{Introduction}

$\mathbf{T}$ he fundamental role of U.S. nuclear weapons in underwriting national security and global stability has not changed even though the nation has taken bold steps to reduce the global nuclear danger. The most dramatic reduction of the nuclear threat was achieved with the signing of the START I (1991) and START II (1993) agreements, as the U.S. and Russia committed to large reductions in their nuclear stockpiles. (Since the breakup of the Soviet Union, Russia and successor states have also signed the START I treaty.) In addition, the U.S. unilaterally halted the development and deployment of new nuclear weapons systems in 1992 and took a number of deployed weapons off alert. The U.S. also began closing elements of the nuclear weapons production complex that would no longer be needed for the smaller stockpile of the future.

\section{The Goal of Stockpile Stewardship}

In announcing the extended moratorium on nuclear testing, President Clinton reaffirmed the importance of maintaining confidence in the enduring U.S.

nuclear stockpile:

"To assure that our nuclear deterrent remains unquestioned under a test ban, we will explore other means of maintaining our confidence in the safety, the reliability, and the performance of our own weapons."

This statement succinctly summarizes the goal of the U.S.
Stockpile Stewardship Program. Nuclear deterrence continues to be a cornerstone of U.S. national security policy. It is the responsibility of the Department of Energy and its national weapons laboratoriesLos Alamos, Livermore, and Sandia-supported by the other elements of the U.S. nuclear weapons complex, to provide the nation with a safe, reliable, and credible nuclear deterrent.

\section{Underlying Assumptions}

The Stockpile Stewardship Program described here is based on several assumptions and observations:

- Knowledge about the design of nuclear weapons is widespread and impossible to eradicate. Many nations that currently do not possess nuclear weapons could develop the necessary technical capabilities.

- U.S. policy will continue to rely on nuclear deterrence.

- The moratorium on nuclear testing will likely be followed by a comprehensive test ban (CTB); the U.S. must adhere to a CTB while, at the same time, retaining confidence in the U.S. nuclear deterrent.

- There are currently no requirements for the production of nuclear weapons; some essential production facilities and capabilities no longer exist.

- No new weapons are being designed.

- Maintaining confidence in the enduring U.S. stockpile-in its safety, security, and reliabilityis essential for nuclear deterrence.
- The enduring U.S. nuclear stockpile will contain fewer weapons, fewer types of weapons, and weapons that will become considerably older than their design lifetime; this stockpile will require enhanced surveillance and maintenance.

- There will be a continuing need to be able to evaluate, recognize, and correct problems that may arise in the stockpile.

- There will be no requirements for modernization to improve military characteristics, but there will be a continuing need to enhance safety and reliability.

\section{Science-Based Stockpile Stewardship}

What are our options for achieving the goal of the Stockpile Stewardship Program-namely, providing the nation with a safe, reliable, and credible nuclear deterrent-given the assumptions stated above? Clearly, the previous methods used by the U.S. are not an option, considering the cancellation of all new weapon development programs, the elimination of essential production capabilities, and the moratorium on nuclear testing.

One possibility is to retire weapons once they reach their designed lifetime. Unless new weapons are produced to replace the retired weapons, the stockpile will shrink and eventually be eliminated. Clearly, this would not be acceptable unless the world situation and national security policy changes so that a nuclear deterrent is no longer needed. 
Another possibility is to routinely replace aged weapons with weapons remanufactured or replicated to the original specifications. However, the elimination of a number of critical production facilities and capabilities (the result of recent downsizing of the nuclear weapons production complex), coupled with the continued evolution of commercial technologies, renders this option difficult and in some cases infeasible over the long term. (See "Remanufactuing and Replicability," p. 6.)
A better option, we believe, is to evaluate and select appropriate responses to specific situations-whether to remove a weapon system from the stockpile, replace with replicate weapons, or replace with weapons incorporating modifications to extend their lifetimes,

\section{Remanufacturing and Replicability}

It is often argued that we should simply remanufacture or replicate a nuclear. weapon according to the original design Spectications when a serious stockplle aging problem is discovered. Whille replication of a defedtive weapon component may be one way to itx a problem. replication is never exact and nay not be easily achieved, cost effective, or desirable.

Problems in replication result from the changes in technology and in health and safety standards that have occurred in the intervening years. Most of these technology changes produce better prod hiets or provilde improved performande. Changes also oceur because U. S. Industry intro duces new prodiulet lines and discontinues others.

Many of the materials used in nuclear weapons are chemically reactive, and denemical incompatibilities among weapon components can lead to material changes that could affect weapon safety, lifetime, our perfommance. In the past decade or so, changes in materinls tedhology have been extensive Some compounds found to be carcinogenic are no longer prodiuced and other changes in materials are nade as a result of econemics and product improvements in the Com: mereial sector. IIIgh explosives in particular are difficalt io replicate Some of the production processes for an older explosive camnot be repro: dured because of current envirommental, health, and safety regulations. Replacing an older explo: sive with a new composition is a signilicant diesign change that in the absence of nuclear test? ing: would be difficalt to certify with confidence: identical rephlication of a troublesome system would invite the recurrence of the trouble. The fact that all modern safety and secunity fea: tures are not present in older systems is note: worthy. Exact replication of a weapon system would predude modifications to the design or fabrication of a weapon despite compelling economic factors or saffety and seccurnty consid: erations:

The nudear weapons in the US stockpile were designed using the nost durable and easyto-manufacture components that would do the: job. Although past designs could, in principle: be replicated with varying degrees of difficulty: depending on when the weapon entered the stodkphle, construction of a device to a speceitic set of bluephints is not sumigtent to guarantee proper performance. Even if all the onginal naterials could be obtained today. we have found through experience that it simply is not possible to fully specify all the subtle manufac turning criteria that affect complex systems like nuidear weapons. It is also not feasible to fully capture, for an indefinite lime, the manufactur ing processes and practices. uised to. produce a nuclear weapon system. This sitwation is particulanly true when we must deal with older tedh nologies for which the scientific expertise is dis: persed and is partidularly troublesome when we cannot use nudlear testing to verify weapon per fromance with the replicated product. (For addi tional discussion on the dhallenges involved in remanufiacturing; see Section VI. 
correct a problem, or substitute a safer or more reliable component. This approach requires an in-depth understanding of nuclear weapons based on fully researched and documented scientific knowledge of these weapons. Such a science-based Stockpile Stewardship Program will provide the scientific basis and practical know-how to ensure the safety, security, and reliability of the U.S. nuclear stockpile without nuclear testing.

\section{Stockpile Assurance}

Crucial as the Stockpile Stewardship Program is to national security, this program alone is not sufficient to ensure confidence in the safety and performance of the U.S. stockpile. A credible U.S. nuclear deterrent requires both the Stockpile Stewardship and Stockpile Management Programs. Figure I-1 shows the division of responsibilities between these two complementary and integrated programs. Generally, the Stockpile Stewardship Program is responsible for research and technology related to evaluting and assessing nuclear weapons and for providing the technology support required for stockpile management. The Stockpile Management Program is responsible for the hands-on functions involved in maintaining the stockpile.

\section{Constraints on Nuclear Weapons}

The issues and problems involved in stockpile stewardship arise, in large part, from the constraints placed on nuclear weapons during their design and manufacture. To fully understand this point, let us briefly review the process that was followed for developing and producing nuclear weapon systems. (See "Nuclear Weapon Development Phases," p. 8.)

In the past, once the need for a specific nuclear weapon was formally approved by the President of the United States, the Department of Defense (DoD) drew up a specific list of teristics for the new weapon system and nuclear warhead. The Department of Energy (DOE) weapons design laboratories (Los Alamos, Livermore, and Sandia) submitted candidate warhead designs for the new system to the DoD. Sometimes it was possible to meet the requirements by modifying warheads from existing systems, but other times a new warhead was required.

In the event that the specified characteristics led to design requirements or military charac-

conflicts, tradeoffs were made to achieve the high-priority items while minimizing the degradation of the competing, lower-priority characteristics. Typical tradeoffs involved:

- Warhead yield vs weight: a higher yield generally requires the use of more nuclear material, which conflicts with the need for a lightweight, long-range warhead. - Safety vs size: enhanced safety calls for the use of insensitive high explosive and fire-resistant subassemblies (e.g., pits), which increases the size and weight of the warhead.

- Nuclear safety vs design margin: reducing the amount of special nuclear material (to enhance safety) imposes penalties in terms of the required design precision.

- Nuclear assembly size vs space for nonnuclear components: reducing the size of the nuclear assembly (to fit a small carrier, for example) usually requires the use of conventional instead of insensitive high explosive, which degrades nuclear safety, whereas reducing the space available for the nonnuclear components (by

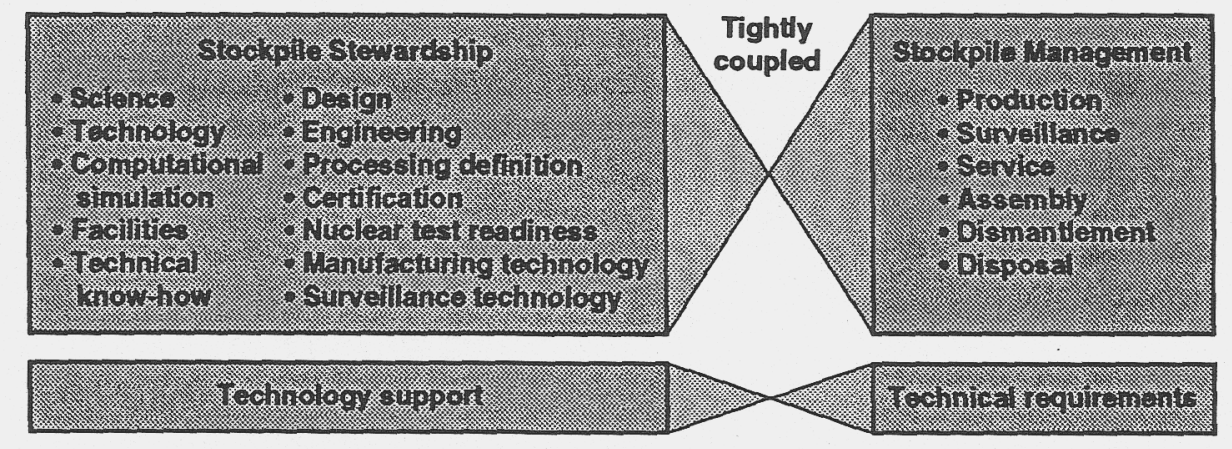

Figure |-1. Stockpile assurance requires both stockpile stewardship and stockpile management. The two programs are tightly coupled, with the Stockpile Management Program providing the technical requirements and the Stockpile Stewardship Program providing the necessary technologies and expertise. 
permitting a larger nuclear assembly) usually requires the use of miniaturized parts for electrical detonation safety and use control that are more costly and technically challenging than full-sized parts.

- Enhanced electrical nuclear detonation safety and use control vs system simplicity: the increased complexity needed to provide improved safety and use control makes system integration more challenging.

Inevitably, such tradeoffs lead to compromises in the military characteristics, creating problems and challenges for long-term stockpile stewardship. Some problems are known at the outset,

\section{Nuclear Weapon Development Phases}

If the need for a specific nuclear weapon is romally approved by the President of the United States, the Department of Energy. (DOE) and Department of Deterse (DoD) coordinate their sched: ulles for its development Joint DoD / DOE Thases. 1 Uhrough 5 have typically required eight to ten y ears of effort. These phases represent a progressive narrowing of options callminating in the delivery of a warhead to the Dod P I hases 6 and 7 encompass the stockpile life of the weapon, which may last several decades: and the eventual retirement and dismantling of the warhead:

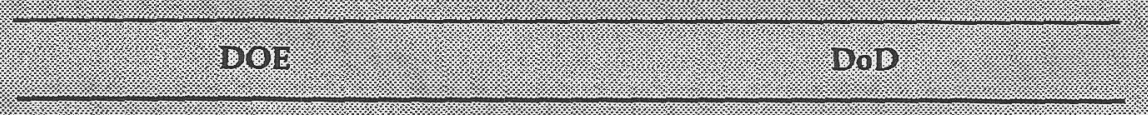

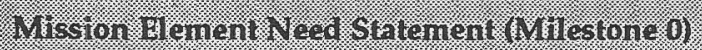

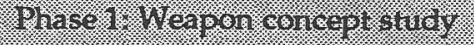

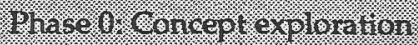

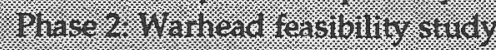

\section{IRMB B * Milestone in}

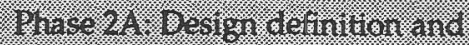

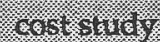

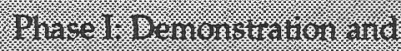

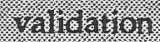

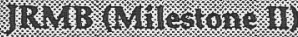

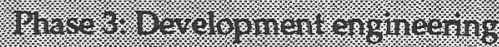

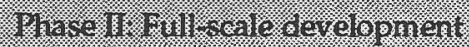

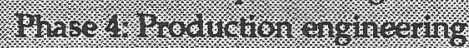

\section{JRVB Whilestone in}

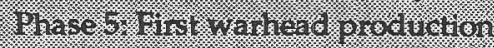

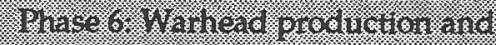

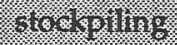

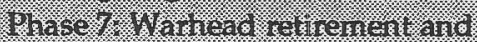
dismantement

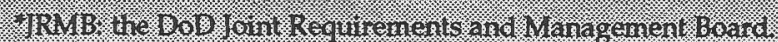

and the stockpiled weapons are monitored specifically for those problems. Other problems are unexpected and are discovered as a result of random sampling and surveillance of the stockpile. Still others are revealed through improved computational modeling; for example, new, threedimensional calculations may reveal problems that could not be detected with the two-dimensional codes available when the weapon was designed.

The adverse effect of aging was one factor that had to be balanced against other military characteristics in the design of nuclear weapons for the U.S. stockpile. If long stockpile lifetime had been a higher priority, it is likely that the warhead designs would have been different. The warheads probably would have been larger and heavier and would have contained more nuclear material; enhanced safety and security features that tend to increase warhead sensitivity to aging might either have been designed to reduced specifications or made more robust (i.e., larger, heavier, and more costly).

\section{Stewardship Responsibilities}

The ability to identify, evaluate, and correct problems that arise with the U.S. stockpile depends on the extent and depth of our knowledge of nuclear weapons. In the past, the severity of a problem or the effectiveness of $a$ "fix" that involved the nuclear assembly could be determined with a nuclear test. If the problems were severe enough, a 
new warhead or weapon system could be developed. With these options no longer available, stockpile stewardship must rely almost exclusively on a sciencebased understanding of nuclear weapons.

Some aspects of weapon design and development have relied on a science-based strategy for years-namely, hardening weapon components and systems against hostile radiation and shock environments of postulated enemy defensive systems as well as determining weapon safety when exposed to any credible abnormal environments. Since it is impossible to know the details of possible defense threat environments, it has been necessary since the 1960 s to conduct science-based engineering development programs for both the military carriers and their nuclear weapons so that they could be predicted to survive hostile environments and function properly. Likewise, since it is impossible to physically simulate many possible accident or other abnormal environments, safety assessments have relied extensively on scientific understanding. These design and development strategies must now be extended to all aspects of nuclear weapon science, engineering, and testing.

\section{Maintaining Confidence in the Enduring Stockpille}

As stated earlier, maintaining confidence in the stockpile means ensuring that the stockpiled weapons are safe and secure and will function as designed should they ever be used. (See "Features in the U.S. Nuclear Stockpile," p. 10.) Many of the issues of concern-relating both to safety and performance-are the result of material changes that occur with age. These issues become much more important as the stockpiled weapons age beyond the lifetime for which they were designed and as the stockpile becomes smaller and less diverse and therefore more susceptible to common-mode failures.

The complexity of these issues arises from the complexity of the physical phenomena that take place in a nuclear weaponexplosion, implosion, mixing of materials, radiation transport, thermonuclear ignition and burn, etc. No technique other than underground nuclear testing is capable of demonstrating nuclear weapon performance at full scale or certifying systemlevel hardness of a weapon to ionizing radiation. With the moratorium on nuclear testing, we must rely on advanced computational modeling and nonnuclear experimental techniques for predictions and data as well as on our cumulative technical know-how to make valid inferences for physics regimes that are inaccessible with current experimental methods.

With few exceptions, the moratorium on nuclear testing does not affect our ability to assess the reliability of nonnuclear components. However, the smaller, less diverse, and aging enduring stockpile presents new challenges for maintaining confidence in its reliability and safety. Many of the nonnuclear components required to turn a nuclear assembly into a reliable, safe, integrated weapon system are highly specialized. As a consequence, lifetimes of individual components are often uncertain. On the basis of historic stockpile data, actionable defects (concerns for possible functional failure based on observations or tests) are expected to occur every few years for each weapon type throughout its stockpile life. All types and categories of nonnuclear component are found in the historical failure database. Since the weapons in the enduring stockpile are likely to age beyond their design lifetime, as well as beyond our experience base for weapons lifetimes, the rate at which defects occur may increase significantly.

Clearly, we must be prepared to replace any component with a functional equivalent for as long as the weapon remains in the stockpile. One-for-one replacements for many of the components by remanufacture to the original specifications cannot be assured; a technology may become obsolete or a supply base of materials or components may become extinct or be interrupted for long periods of time. In order to ensure cost-effective replacement of specialized components, we will make wider use, to the extent possible, of model-based design, proven by performance simulations and coupled with predictive models of the manufacturing processes. This approach will require significant advances in scientific understanding, but, in the longer term, will result in cost savings due to a reduced cost of maintaining production capability. In addition, because interactions between components can 
affect system performance in unpredictable ways, a scientific basis is needed for certifying the acceptability of replacement components. Here again, system-level models will underwrite the required capability.

Our technical know-how is also the only way we now have for evaluating many crucial issues, including:

- The severity of age-related material changes discovered through routine stockpile surveillance.
- The severity of unexpected effects discovered with improved computer models. - Whether new technologies or minor changes compromise the radiation hardness of a weapon.

- Whether retrofits--to correct safety issues or to modify a weapon for a different delivery system-will indeed function properly.

- Whether new technologies (for example, for safety improvements) can or should be incorporated in a stockpiled weapon system.

It is clear that in order to address these issues, we must improve our nonnuclear experimental capabilities, extend our computational models, and preserve and enhance the base of technical knowledge that is unique to nuclear weapons. Our existing nonnuclear test facilities were developed to complement nuclear testing; these facilities need to be upgraded and new

\section{Features in the U.S. Nuclear Stockpile}

US. nudear stockplie contains weapon systems with various features for safety. Security. and use control.

la
l

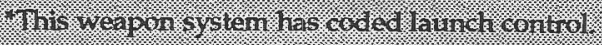


capabilities need to be designed and built. These capabilities are shown in Table I-1. Improved hydrodynamic test facilities, the Los Alamos Neutron Scattering Center, and the proposed National Ignition Facility will be particularly important, providing experimental data that cannot otherwise be obtained. Other, smaller facilities will be required (see Table I-2).

Computational modeling relied heavily on nuclear test data for validation and calibration. Now, these models are our only means of estimating the performance of a weapon at full scale. Advances in high-performance computing are needed so we can simulate weapons in three dimensions with models of all important physical processes. Advances are also needed to develop the essential weapon-system-level models so we can assess weapon safety when exposed to environments that are impossible to physically simulate or to a wider range of scenaries than is affordable to test. In order to exploit the improved performance possible with massively parallel processing, we must write new codes and restructure existing codes (some of which have taken decades to develop). This will be an immense but essential undertaking.

\section{Hedging Against the Uncertain Future}

The reduction in tensions between the U.S. and the former Soviet Union, accompanied as it has been by mutual agreements and unilateral decisions to reduce nuclear stockpiles, sets the stage for globally reducing the nuclear danger. However, the future is uncertain, both on the political front and on the scientific front. Many countries are striving, openly or covertly, to develop nuclear weapons. Others already have, and still others will likely obtain weapons in the futurewitness the recent headlines about China's nuclear tests and North Korea's acknowledged nuclear weapon development activities.

It is also impossible to accurately foresee technical problems that may arise with the U.S. stockpile or to predict the nature of future scientific breakthroughs. Future stockpile problems could require the retrofitting or replacement of entire weapon systems. Future scientific breakthroughs (even inadvertent ones) could lead other countries to new types of weapons, and our security could depend on our being able to analyze the implications of these new weapons and respond appropriately.

Clearly then, stockpile stewardship requires that we retain a nuclear weapon manufacturing capability. This capability will be needed to provide the scheduled replacements of limited-lifetime components (e.g., tritium-containing components). It will also be required to meet the need for unscheduled replacements of age-related defective parts and to provide for unexpected safety, security, or reliability "fixes." (See "Remanufacturing and Replicability," p. 6.)

With the end of the U.S.Soviet arms race, the DOE nuclear weapons production complex is being downsized, and a number of facilities have been permanently closed. In addition, some of the processes for making weapon materials and fabricating weapon parts are no longer acceptable in light of new environmental and safety regulations. As a result, the three weapons laboratories-Los Alamos, Livermore, and Sandia-will likely have to serve as the repository for manufacturing and materials technology for nuclear weapons. We must also develop new, environmentally benign, and safer processes so that the necessary technologies are in place should a retrofit or rebuild be necessary. The factory of the future will be tailored to low-volume production; it will use modernized design and production practices, and it will be closely integrated with U.S. industry.

\section{Preserving a Unique \\ Knowledge Base}

The knowledge base relevant to nuclear weapons must be maintained and enhanced. At the most fundamental level, stockpile stewardship rests on scientific and technical judgment and skill. Most of the people who have actual experience in nuclear testing and in putting new weapon systems into the U.S. stockpile will retire in the next ten years or so. The scientists and engineers responsible for stewardship of the stockpile from that point on will have had no hands-on experience in nuclear testing and perhaps in weapon development. The current nuclear know-how must be passed on to the next generation of "stockpile stewards," and this unique knowledge base must be enhanced and 


\section{Introduction}

extended so that these "stewards" can make scientifically based assessments of weapons issues. This knowledge base is essential throughout the life of the nuclear stockpile, including dismantlement and disposition of the nuclear materials.

We cannot be content with our scientific and technical achievements; new discoveries will be made, whether or not the
U.S. has a nuclear weapons program. Research into new areas of physics, materials, engineering, computations, etc., is essential if the U.S. is to stay at the forefront of science and technology and

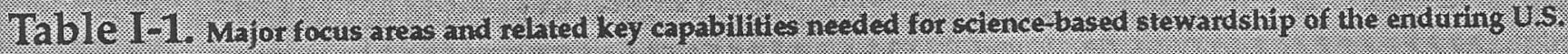
nuclear stodikpillo.

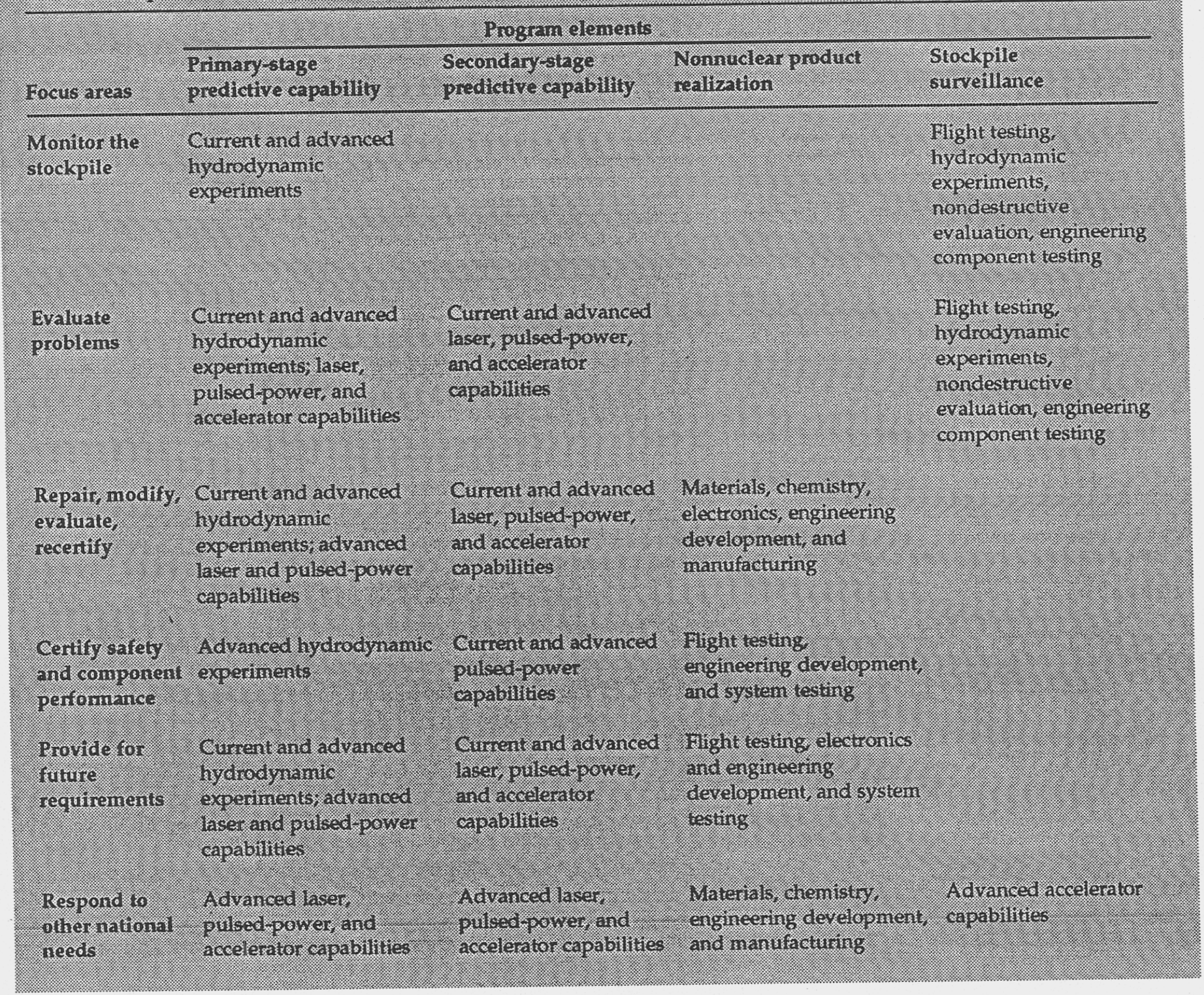


protect against being caught short in the face of technological surprise. Neither can the nation risk a nuclear accident through inattention or the loss of crucial know-how.

\section{Partnership with U.S. Industry}

To meet the future need for replacement and upgraded nuclear weapon components at an affordable cost, U.S. industry must be integrated into the product-realization processfrom technology development to assembly. Most important, we must greatly increase the integration of the nuclear weapon technology and manufacturing bases with those of the private sector. To this end, we must eliminate historical barriers between the nuclear weapons complex and U.S. industry and implement new business practices in the weapons laboratories and plants.

Although the nuclear weapons complex will have to maintain the capabilities to manufacture some weapon parts and components, the use of commercial parts and private sector technology must be expanded if costs are to be held at an affordable level.

The Stockpile Management Program will shoulder the bulk of the effort required, and reap most of the fruits of this effort, to integrate the technology and manufacturing bases of the nuclear weapons complex and the private sector. However, the

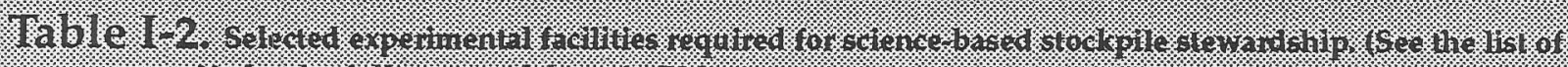

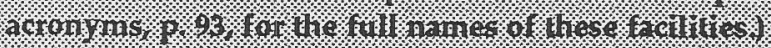



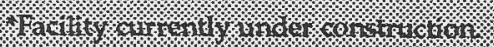


Stockpile Stewardship Program will be very much involved, developing the technologies needed to effectively implement the integration.

The Technology Partnership Program, an element of the Stockpile Stewardship Program, is one of the mechanisms that will be used to integrate the nuclear defense and private sector technology and manufacturing bases. This program is set up to support cost-shared partnerships with U.S. industry to develop mutually beneficial technologies and capabilities. These partnerships will help maintain the core capabilities that form the foundation of the Stockpile Stewardship Program and will provide private industry with new commercial processes and products.

\section{Roadmap for the Report}

The strategy for sciencebased stockpile stewardship, as described in this report, is an integrated program drawing on the complementary strengths of the Los Alamos, Livermore, and Sandia laboratories. In the sections that follow, we first provide a brief description of the operation of a nuclear weapon (Section II). We then discuss the issues of concern and the programmatic thrusts to address these concerns for nuclear weapon primaries and secondaries (Section III), for the nonnuclear components of a nuclear weapon (Section IV), and for integrating the nuclear and nonnuclear components into a weapon system (Section V). We also describe the issues of concern and programmatic thrusts related to nuclear weapon materials and manufacturing technology (Section VI), stockpile surveillance (Section VII), computational modeling and numerical simulation (Section VIII), and test readiness (Section IX). We briefly review the contributions of a science-based Stockpile Stewardship Program to other areas of national concern, including technology partnerships with industry (Section $X$ ) as well as nonproliferation, advanced nonnuclear defense technologies, fundamental science, and technologies for commercialization (Section XI).

\section{Recommendations for the U.S. Stockpile Stewardship Program}

The Stockpile Stewardship Program must address a spectrum of technical issues, and these issues, in turn, drive the necessary programmatic activities. Below, we summarize the major programmatic thrusts for stockpile stewardship. Each topic is discussed in greater detail in the sections that follow.

Nuclear Components: Primaries - Implement improved methods (hydrodynamic and hydronuclear, if authorized by the President) to define implosion performance and assess weapon reliability and safety.

- Develop improved computer codes and improve our understanding of materials behavior and the physics involved in primary boosting so that hydrodynamic and hydronuclear data can be extrapolated to full primary performance to assess aging and support certification.

\section{Nuclear Components: \\ Secondaries \\ - Construct and utilize experi- mental facilities that can pro- vide conditions relevant to sec- ondary performance. \\ - Develop computer codes incorporating improved physics models that can predict the effects of subtle aging phenome- na on secondary performance.}

\section{Nonnuclear Components}

- Provide the scientific basis for accepting new materials or components that differ from the originals.

- Improve our understanding of aging-related effects on nonnuclear components to predict failures and to design replacement parts with longer lifetimes.

- Upgrade the technologies for use control implementation and electrical nuclear detonation safety.

\section{System Integration}

- Develop systems-level models for assessing safety, thereby reducing reliance on physical testing.

- Maintain active systemdevelopment programs focused on improving safety or reliability or reducing lifetime cost that are technically challenging. 
- Develop engineering test capabilities to understand the effects on safety and reliability as weapon systems age beyond their intended lifetimes.

Manufacturing and Materials Technology: Nonnuclear Components

- Work with U.S. industry to develop dual-use materials and processes (the virtual enterprise) to reduce the cost of process development and product production and to provide a steady supply base.

- Implement virtual prototyping (complete simulation of product design, performance, and manufacturing before any hardware is produced), concurrent engineering, and agile manufacturing practices for nonnuclear components.

- Use process models and sensor-based process control (smart processes) to ensure affordable quality products in small lot sizes with intermittent production.

- Develop processes for nonnuclear components that are more environmentally acceptable and safer.

\section{Manufacturing and Materials Technology: Nuclear Components}

- Maintain a capability to fabricate, dismantle, and process nuclear-related materials and components using technologies that minimize waste and environmental hazards, improve worker and public safety, and are cost-effective and reliable for small-lot fabrication.

- Evaluate technologies for producing and extending the supply of tritium and select preferred option.

- Improve our understanding of materials properties and fabrication processes as they relate to remanufacturing weapon components.

- Develop technologies for the environmentally sound disposition of nuclear components and weapon materials.

\section{Stockpile Surveillance}

- Move from routine preventive maintenance and periodic inspection schedules to self-diagnostics for maintenance on demand.

- Develop noninvasive imaging techniques to assess the consequences of age-related defects in the internal structure of nuclear assemblies without disassembling the weapon.

- Perform hydrodynamic experiments to verify implosion characteristics of primaries and assess the effect of age-related changes on weapon performance or safety.

\section{Computational Simulations}

- Develop improved theoretical models of important physical processes.

- Procure and help drive the development (in partnership with U.S. industry and other government agencies) of massively parallel processors, large datastorage systems, and high-speed networks.

- Develop application software to exploit parallel machine architectures and system software to support an integrated user environment with improved datamanagement tools.
The Nevada Test Site and Test Readiness

- Perform aboveground and underground experiments to address stockpile stewardship issues and exercise nucleartest-related skills.

- Conduct test and demonstration activities at the Nevada Test Site for other users to exercise, utilize, and support the testing and experimental infrastructure.

- Identify and interview persons possessing critical nuclear testing knowledge and record the information in a consistent and retrievable format.

\section{Technology Partnerships with} U.S. Industry

- Develop standards for assessing the value of projects and programs to both the nuclear weapons program and U.S. industry.

- Fully integrate industry partnership activities with those that directly support stewardship and maintenance of the enduring stockpile.

\section{Overall}

- Improve our understanding of the physics involved in all stages of the operation of a nuclear weapon.

- Improve our understanding of materials aging and compatibility and their effects on weapon safety and reliability.

- Improve current nonnuclear test techniques and facilities and develop new techniques and facilities to compensate for the absence of nuclear testing. 
- Improve our manufacturing and materials technology, stockpile surveillance, and computational modeling capabilities.

- Preserve the technical knowhow unique to nuclear weapons.

\section{Planning in a Rapidly Changing Environment}

This strategy was developed in a rapidly changing and somewhat unpredictable environment. It is based on our best assessments of the current situaltion and the desired objective and on some reasonable but uncertain assumptions about how the world will evolve as the plan moves forward.

The strategy outlined in this report addresses our five- to ten-year vision of science-based stockpile stewardship and its essential components. Instead of addressing each uncertainty as it arises in this document, let us acknowledge up front that there are a number of important unknowns that must yet be resolved. For example, we assume the continued existence of three weapons laboratories, but that could change as a result of the current Galvin Committee study. Also, the scope of a comprehensive test ban, which is still unresolved, will affect the type of experiments that can or cannot be conducted. In addition, ongoing negotiations on the extention of the Nuclear NonProliferation Treaty (NPT) may affect what facilities are built and what experiments are conducted. In this regard, the Secretary of Energy has recommended that we do not conduct or prepare to conduct hydronuclear experiments during the current moratorium on nuclear testing and that the decision about conducting hydronuclear experiments be delayed until after the NPT Extension Conference next spring. Finally, there is no way to predict the funding levels from Congress or to anticipate lawsuits or other external interventions that may be directed at the program. Although modifications will be made to the program as issues arise, we expect that the vision and strategy of the Stockpile Stewardship Program will remain essentially unchanged.

\section{Looking to the Future}

Nuclear weapons and their consequences are too important to be managed by second-rate science and technology. The national imperative associated with nuclear weapons has attracted and maintained outstanding personnel in these programs through the last five decades. Although the Cold War is over and the stockpile is being rapidly reduced, the assurance of nuclear weapon safety and reliability remains as important as ever. Science-based stockpile stewardship is a major technical challenge that can attract and retain the high-caliber people in the wide range of technical areas needed to maintain confidence in the stockpile. Having the trained, experienced judgments of these outstanding scientists and engineers is an absolute necessity. The science-based Stockpile Stewardship Program is designed with this goal in mind. 


\section{Introduction to Nuclear Weapons and Weapon Physics

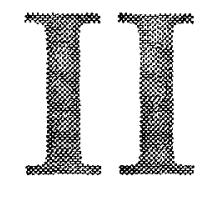

$\mathrm{T}$ he design for the nuclear weapon detonated at Hiroshima was never tested, yet it worked. The weapons of the U.S. nuclear stockpile today are very different-much more sophisticated and complex and not as robust. They were designed to fit the physical constraints of specific delivery systems and often were optimized for maximum yield-to-weight ratio; in addition, they include sophisticated safety and security features.

With the planned reductions in the U.S. stockpile, certainty in the performance of individual weapons and complete weapon systems is a vital concern. This certainty was previously demonstrated by nuclear tests. The science-based Stockpile Stewardship Program is designed to enable the U.S. to continue to ensure the safety, security, and reliability of the aging, smaller, and less diverse enduring stockpile without nuclear testing.

\section{Operation of a Typical Nuclear Weapon}

\section{Modern thermonuclear} weapons consist of two stages, the primary and the secondary, plus a radiation case that channels energy from one to the other. The primary stage functions by compressing a shell of fissile material (principally plutonium) with a high-explosive charge. The initial subcritical assembly of fissile nuclear material, called a pit, is symmetrically imploded. In this subcritical phase, there is no nuclear yield. The overall density increases and becomes high enough that the nuclear material reaches a supercritical state. At the proper time, neutrons from a neutron generator are injected into the pit to initiate exponential growth in the neutron population and energy production. In a boosted primary, a cavity in the center of the pit is filled with deuterium and tritium gas (DT). During implosion, this gas is compressed and heated until it undergoes fusion, and neutrons from the fusion process flood the compressed pit. This pulse of additional neutrons in the supercritical pit greatly increases or "boosts" the fission yield.

Most weapons in the stockpile have a thermonuclear secondary stage. The last operational phases of a thermonuclear device involve the implosion and ignition of this stage. Radiation from the hot exploding primary is channeled by the radiation case to the secondary. This compresses and ignites the secondary, which produces fusion energy from the lithium deuteride fuel. Fusion neutrons are captured by lithium deuteride salts contained in the secondary, producing tritium that subsequently undergoes fusion reactions with deuterium in the fuel.

This description is highly simplified. The complexity of the physical processes involved is staggering. We know that our scientific understanding of many of these processes is incomplete. Even if we had a complete and detailed understanding of the physics of nuclear weapons, an exact mathematical description of the various processes and their interactions would exceed the capabilities of today's supercomputers as well as those appearing on the immediate horizon. To accommodate computer limitations, we must make approximations to the physics in our evaluations of nuclear weapon performance. However, such approximations result in uncertainties in the accuracy of the results.

In years past, nuclear testing provided a pragmatic solution to this limitation. Under the current moratorium on nuclear testing, and to sustain confidence in the stockpile under a potential comprehensive test ban, we must find other means of improving our predictive capability for nuclear weapon performance. Since weapons are likely to remain in the stockpile for lifetimes well beyond our experience base, these predictive capabilities must include age-related effects, which are currently not well understood. The science-based Stockpile Stewardship Program will accomplish this through a combination of advanced computational capabilities and an enhanced program of nonnuclear experiments.

A Science-Based Approach to the Physics of a Nuclear Weapon

Although no laboratory experiment can duplicate the amount of energy released by a 
nuclear weapon, many of the physical conditions relevant to nuclear weapons can be created in a laboratory setting.

For primaries, the hydrodynamics of the implosion process, short of criticality, can be accessed experimentally with full-scale assemblies using mock nuclear material. Current hydrodynamic test facilities can access this precritical physics regime. However, they were designed to complement nuclear tests and, by themselves, are not adequate for the task. We must modify existing hydrodynamic facilities and develop new ones that can provide us with much more information on the spatial and temporal evolution of primary components in the precritical phase. Extrapolating beyond the hydrodynamic tests to the full performance of a primary will require more than better hydrodynamic data. We must also obtain a better fundamental understanding of the essential physics and material properties for primaries. This is a critical part of the science-based Stockpile Stewardship Program.

Improving our predictive capabilities for evaluating secondaries will be more difficult. Without nuclear tests, we can never directly observe actual operation of the secondary stage.
Therefore, we must improve our understanding of the relevant physics with better computations and new nonnuclear experiments, facilities, and techniques. There is no single, unique experimental capability that can replace nuclear testing in assessing the reliability of secondaries. Rather, we must rely on a set of complementary techniques.

For both primaries and secondaries, our goal is to make our computational tools as good as they must be, validated by experiments, to address any and all problems in the stockpile as they occur. 


\section{Nuclear Components}

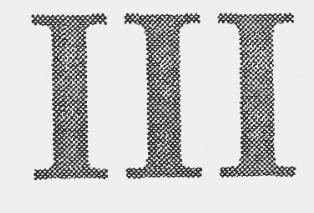

$I^{\mathrm{n}}$ $\mathrm{n}$ the absence of nuclear testing, nuclear weapon performance will have to be inferred from theoretical calculations and nonnuclear experiments.

Research must focus on eliminating existing weaknesses in the predictive accuracy of nuclear design codes and on assessing the effects of age-related material changes on weapon performance. Enhanced experimental capabilities and facilities are needed that can provide the necessary data to assure confidence in our calculations. No single facility, of course, can replace nuclear testing, but the right set of facilities can help ensure the continued health of the stockpile and confidence in the nation's nuclear deterrent.

Some of the issues of concern are specific to primaries or secondaries. Other concerns, such as the need to assess the effects of age-related changes, to improve our predictive capabilities for weapon performance, and to acquire new experimental facilities to compensate for the moratorium on nuclear testing are common to both.

\section{Primaries}

The primary of a nuclear weapon is a crucial subsystem for weapon reliability and safety. Without proper primary-stage function, the secondary will not work. The primary also contains the main high explosive and the plutonium that comprise the principal safety concerns.

The importance of the primary to the continued reliability of the stockpile is related to components that are subject to degradation with age, and to the critical role of boosting, which, if inadequate, can result in a dramatic failure of weapon performance. In the past, there have been several examples of problems in the primaries of stockpile weapons, due to aging or the discovery of design or engineering weaknesses, that have required fixes or retirement to maintain stockpile reliability. Much effort over the years has been directed toward assuring that the current stockpile is safe.

The primary is a critical element relative to the safety of a weapon because of the potential

\section{Primaries}

Key Issues

- Confidence in the stockpille is based to a large extent on ensuring the reliability and safety of the primary.

- Vaterial degradation and imperfections caused by aging can profoundly affect primary performanice and safeety.

- We must be able to accurately predict implosion performance. boost, yield, and response of the primary to aging effects or changes introduced in the process of correcting a primary prob. lem. This understanding will allow us to evaluate the need for remanufacture and to certify that a primary problem has been corrected.

- We need to be able to accurately predict the responnse of the prinary to abnormal enviromments.

- Current capabilities are not sufficient to accurately evaluate and certify primaries:

\section{Major Thrusts:}

- Improve hy arodynamic testing capabilities to adequately. define implosion perfommance and assess weapon reliability and safely.

0 ?erform hy dronuclear experiments, If authorized, to assess weapon rellability and safety.

- Better understand how materials behave in primary-stage operation and the complex physics involved imboosting a prit mary. Improved understanding will allow us to more confident ly extrapolate hydrodynamic and hydronudlear data to primary performance.

Improve scientific understanding and models to assess the
ing safety of primaries in new creaible abnommal enviromments. of Improve manuifacturing and materials technology stockpille surveillance, and computational modeling capability (see Sections VI, VII, and VIII). 
for accident-induced detonation of the high explosive and dispersal of plutonium.

Increasingly sophisticated calculations and experiments contribute to an evolving understanding of safety and to improving confidence in predictions of weapon response in abnormal environments. Postdeployment testing and modifications to assure one-point safety relative to accidental nuclear yield have been necessary in the past.

Requirements for fire safety have arisen from several accidents involving nuclear weapon delivery systems. Various levels of detonation and fire safety are incorporated into stockpile weapons.

\section{Issues of Concern}

Issues of concern related specifically to primaries have to do with proper functioning of the primary in its various stages of operation, the effects of aging-related changes on primary components, and the need to certify "remanufactured" primaries and components, among others.

\section{- High-Explosive Detonation} and Burn. After the detonators are triggered, a detonation front begins in the main charge. Highexplosive (HE) burn is affected by the type of explosive and its chemistry, grain size, impurities, manufacturing method, temperature, and assembly gaps. Our calculational models of $\mathrm{HE}$ burn and performance, including both burn propagation and $\mathrm{HE}$ equation of state, are generally empirical and do not fully describe the underlying physics. These models can be normalized to hydrodynamic experiments and are relatively good for a limited set of circumstances but can be unreliable in predicting $\mathrm{HE}$ performance for others.

Detailed reactive-burn models that attempt to describe the underlying physics are available but are not used for all calculations, in part, because they are quite costly to run and are still incomplete models. (Such models are used, however, in many accident simulations where physics can be important to predicting $\mathrm{HE}$ detonation.)

The radiation case, which helps channel radiation energy from the primary to the secondary after primary explosion, begins expanding as the detonation front passes. The behavior of the case depends on material grain size, manufacturing methods, age, impurities, corrosion, pressure, strain, strain rate, and external components. Some aspects of this phase of nuclear weapon operation are poorly described by existing models.

- Pit Implosion. The implosion begins when the HE burn reaches the surface of the pit. The pit itself follows a complicated path during implosion. The detailed history of pit implosion begins with a perpendicular shock and horizontal shear in the pit material as a result of high detonation pressures produced by the HE. The details of the response of the pit to the complex driving conditions encountered in primaries (how the material moves, flows plastically, melts, sublimates, or spalls, for example) are also quite complex. Materials behavior within the pit depends on grain size, manufacturing technique, impurities, equation of state, texture, and grain orientation.

Dynamic material behavior is not fully understood and is represented by approximations rather than by fully accurate physics models in our design codes. For example, there is still limited data on the plutonium equation of state near melt, the melt temperature as a function of pressure, and the behavior of plutonium at high strain and high strain rate.

The shock wave enters the pit, triggering a complex series of interactions. These interactions are parameterized reasonably well empirically but are modeled poorly from a first-principles point of view. The pit is designed to implode radially and converge to a compact shape. However, contact-pin measurements and core-punch $x$-ray images of the surface of imploding pits sometimes show anomalies when compared with calculations.

As the detonation shock traverses the pit, it ejects material from each interface. This ejected material can depend on a number of factors, including surface metallurgy, and is not well described empirically or from first principles. The detailed effects of this ejecta are complex and are not fully understood. This is an active subject of research, and improved modeling tools are being developed. - Boost. The heated and compressed boost gas undergoes fusion. The main effect of this thermonuclear process is to 
generate large numbers of highenergy neutrons that enter the fissile pit material and cause subsequent fissions. These boostinduced fissions generate additional fission yield, "boosting" the yield of the primary.

Typical calculations estimate the observed thermonuclear burn efficiency incorrectly. Mix of material is incorporated empirically in the calculations. There are a number of potential sources and mechanisms for such mixing, including a variety of hydrodynamic instabilities, ejecta from various sources, and turbulence.

- Effects of Aging on Primaries. Age-related changes may occur in any and every component of a nuclear weapon. Although some components can be replaced relatively easily, the nuclear components cannot be readily exchanged without remanufacture and the attendant issues of cost and recertification.

Age-related changes that can affect the primary include:

* Structural or chemical degradation of high explosive leading to changes in explosive performance.

* Migration of highexplosive materials.

* Changes in plutonium material properties (such as ductility and shock heating) with the buildup of impurities inside the metal due to radioactive decay.

* Corrosion of interfaces, joints, and welds.

* Chemical or physical degradation of other materials and components.

These are not hypothetical or theoretical problems, but actual problems that have occurred in stockpile systems and thus can be expected to occur in the future.

Predicting the effects of age-related changes on primary performance or safety stresses our capabilities and understanding. There have been instances in the past where new information (often the result of improved experimental or calculational capabilities) has revealed inadequacies in our understanding. The significance of past problems causes concern about our ability to predict the effects of unexpected changes, especially under different conditions for which our calculations have been benchmarked (i.e., with nuclear tests).

Figure III-1 illustrates the array of technical issues associated with aging. The aging of plutonium is an issue of particular concern. Pits in the enduring stockpile will become older than any plutonium with which we have experience. Plutonium has existed in more than infinitesimal amounts only since the 1940s.

Effects on reliability of the primary will occur mainly through perturbation of the primary implosion and its effect on boosting. Current models of this complex process do not provide believable predictions of such effects.

Safety can be affected by chemical or structural changes in the high explosive or detonators, which may lead to altered response to impact or fire.

Corrosion or cracking may compromise fire-resistant layers. The effect of such changes will be particularly important in abnormal environments (for example, an accident). Again, these are complex issues, which are not fully understood scientifically. Yet we must be able to assess their effect on weapon performance and judge whether (and when) remanufacture is required. - Certifying Remanufactured Primaries. If the effect of aging or the discovery of a defect is serious enough to require the replacement of primary components - the high explosive or pit, for example-- the very act of fixing a problem will raise the issue of assuring that the fixed or remanufactured primary will perform adequately.

A change in a major nuclear component is essentially a redesign, requiring the same special skills and expert judgment needed for a new design. We must be able to ensure that no change (for example, in the detonation characteristics of the high explosive, metallurgy, material properties, or finish) compromises primary performance. (The practical impossibility of ensuring "exact" remanufacture of these components is discussed in Section VI.)

Implosion properties relevant to boost can be modified by relatively small changes in such physical characteristics. For example, is ignition of the boost gas assured? This question and others cannot be directly or completely answered in nonnuclear experiments. In the past, such questions were addressed in a nuclear test.

Certifying proper boosting in a remanufactured primary is 


\section{Nuclear Components}

of particular concern. We have not identified any means other than nuclear testing to assess some of these issues. In the absence of nuclear testing, we will need significant additions to our scientific understanding together with an improved predictive capability for modeling boost in past underground tests and predicting boost-related details of hydrodynamic tests and hydronuclear experiments. Current experiments do not sufficiently define even the nonnuclear phase of primary operation, a necessary precondition for accurately defining the conditions required for boosting.

- Improvements to Meet Changes in Requirements. It is likely that new requirements, probably for increased safety and security, will be placed on the stockpile that could, in turn, require modifications to the nuclear assembly. It is also possible that stockpile weapons will have to be modified to fit a new delivery system. Some requirements may be addressed by fixes with little potential for impact on nuclear performance (for example,

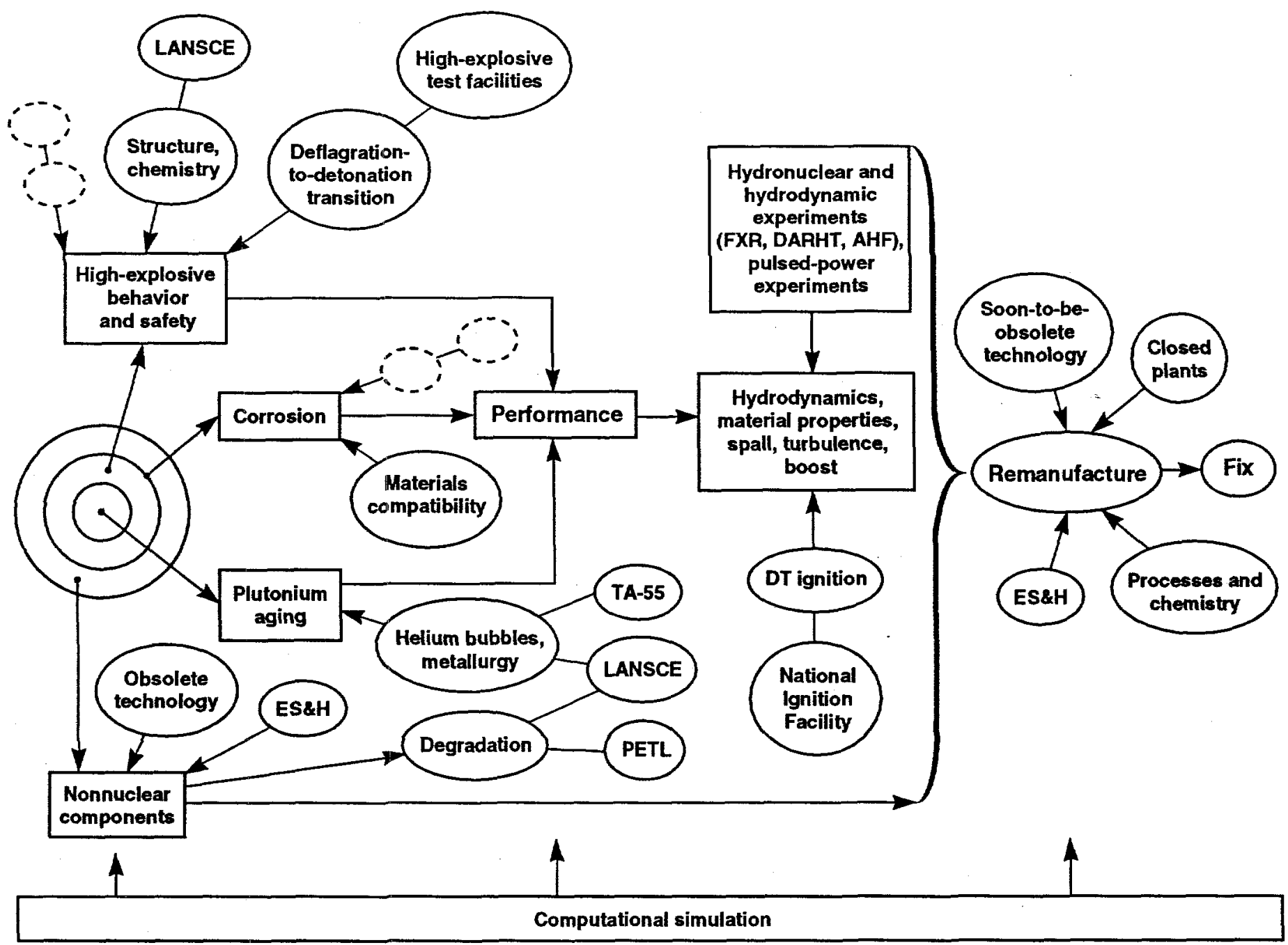

Figure III-1. A wide array of technical issues and experimental approaches are associated with ensuring the safety and reilability of aging primaries. 
changes to the weapon electrical system). Others however could entail a redesign (for example, a requirement for multipoint safety in accidents). As a result, such new requirements could not be entered into lightly, given the safety and performance certification aspects associated with changes in design.

\section{The Program for Addressing these Issues}

Addressing the stockpile stewardship issues regarding primaries requires two basic elements: an enhanced computational capability and comprehensive experimental capabilities.

- Enhanced Predictive Capability for Primaries. Advanced computational modeling for nuclear weapons has made possible increased design sophistication with less testing, which reduces costs, to evaluate nuclear performance. The moratorium on nuclear testing forces a much greater reliance on the accuracy of code predictions and the completeness of code models. Many issues of primary (and secondary) performance-for example, boost-gas fill tubes, one-point safety, and the effect of gaps or voids - are inherently three-dimensional problems. The ability to predict the effects of age-related changes will be crucial to judging whether and when remanufacture is required and to certifying that newly fabricated component replacements do not compromise device safety or performance. However, as noted above, such calculations stress or are beyond our current computational capabilities, and aspects of our theoretical models are limited or inaccurate (see Section VIII).

As we improve our modeling capabilities, we must validate them, to the greatest extent possible, through comparison with the results of past nuclear tests, with the results of new nonnuclear tests, and with the results of experiments on weapon-like assemblies. Improving the archives of nuclear test data and archiving weapon design and engineering experience for each weapon system in the enduring stockpile are important nearterm elements of the Stockpile Stewardship Program.

- New Experimental

Measurements. New experimental measurements are needed to pin down the physical quantities and processes relevant to primaries so we can extrapolate to conditions that cannot be measured directly and thereby predict and evaluate the performance of aging or remanufactured primaries. To this end, we must first define the physics understanding needed to carry us past the last stages of primary function that can be directly measured without a nuclear test-that is, past the calculational "leaping-off point" of hydrodynamic tests and hydronuclear experiments.

The additional experimental data needed include:

* Material properties that affect hydrodynamic and shock behavior for plutonium, uranium, high explosives, and various other materials.

* Neutron cross sections for selected nuclear reactions related to energy release.
* Development over time of the three-dimensional density distributions and distortions of an imploded pit.

* Evolution of mix, including the definition of sources of mix (e.g., ejecta, spall, instabilities), the transport of mixed materials, and distribution of mixed materials.

* The boost process, including the effect of contaminants on burn.

* Behavior of the radiation case.

* Response of the primary

to abnormal environments, including impact, fire, crush, and combinations of these environments.

- Hydronuclear Experiments and Hydrodynamic Tests. New experimental capabilities could compensate for the unavailability of nuclear testing. Two complementary approaches are included in our strategyhydronuclear experiments and advanced hydrodynamic testing. Hydronuclear experiments, if approved by the President, will provide information related to the fission drive necessary for boosting. Advanced hydrodynamic tests will provide detailed information on the implosion leading to boosting, but no direct measure of nuclear energy production.

Hydronuclear experiments can be valuable for two reasons. They can be used to directly address the nuclear detonation safety of a stockpile weapon. They also could provide an important benchmark performance measure. Some modifications 
that alter the configuration or operation of a primary would be required to meet the yield limits associated with hydronuclear experiments. Such experiments would provide direct measurements of compression using neutron multiplication and thus would require less extrapolation to interpret normal performance than is required with hydrodynamic test data.

Improvements in hydrodynamic testing, including an Advanced Hydrotest Facility, are necessary for preserving confidence in stockpile reliability. Hydrodynamic test facilities have long been used as a part of the primary design process and, occasionally, in stockpile assessment. These tests provide measurements in the early, hydrodynamic phase of a pri-mary's implosion. The only tool available for probing the structure of a pit at late times in the implosion is penetrating $x$ radiography. These hydrodynamic tests typically use mock materials.

It is a major challenge to extrapolate from hydrodynamic test data alone to predict the performance and safety of aging or remanufactured primaries. Data from hydrodynamic experiments will contribute particularly to safety measurements and to identifying reliability concerns. In order for these tests to provide useful quantitative information about primary performance, they must be able to measure the detailed, threedimensional (3D) distribution of mass as a function of time. The data would then provide information on the:
* Criticality of the device as a function of time around maximum compression, which would make it possible to predict nuclear safety or the explosion behavior of the pit.

* Detailed behavior of the pit material, which would help in predicting boost.

* Bounds on or, if possible, measurements of the amount of mixed material.

Determining the criticality of the device, for example, requires accurate absolute measurements of density distribution as well as an adequately accurate determination of the time of these measurements relative to peak criticality. Because of a small timing jitter inherent in HE initiation systems, single-flash radiographic measurements are limited in the accuracy to which details can be determined.

To obtain images late in the primary implosion, when the pit is extremely dense, we need flash $x$-ray machines that can provide a high $x$-ray dose, high spatial and mass resolution, and a nearly optimal spectral distribution. To better assess accuracy of our models of primary function, we need a tomographic technique (like a CAT scan) that can provide high-resolution $3 \mathrm{D}$ data taken at several different times during the implosion. A high-quality 3D tomographic "reconstruction" requires several different flash images from different angles (multiple-axis imaging) at each time.

Specifications and technologies are being developed and evaluated for an Advanced Hydrotest Facility that would meet the requirements discussed above; in addition, the facility would allow for fully contained implosions. With this new facility, we will be able to produce a 3D movie of an imploding pit with the detailed information necessary to infer criticality and mix.

The enabling research and development and the construction of an Advanced Hydrotest Facility are anticipated to take nearly a decade, and thus it would not be available before the year 2005. This leaves a crucial ten-year time in which we must rely on hydrodynamic test facilities that currently exist or are already in development.

- Near-Term Improvements in Hydrodynamic Testing. The Flash X-Ray (FXR) facility at Livermore and the PHERMEX (Pulsed High-Energy Radiography Machine Emitting $X$ Rays) facility at Los Alamos are the best currently available facilities for radiography of primary implosion. These facilities are instrumented with a full range of diagnostics, including highspeed optics systems, velocimetry, and contact-pin measurements.

A major improvement to our hydrotesting capability will be provided by the DARHT (Dual Axis Radiographic Hydro Test) facility at Los Alamos. The first axis of this facility is currently under construction and scheduled for completion in 1997; it is being configured as a national user facility. With this new facility, we will be able to do radiographic experiments at a significantly higher resolution and with increased penetrating 
power than is available from existing facilities.

Modifications to Livermore's FXR accelerator (due to be completed in 1996) are being made that will allow double pulsing. With double pulsing, it will be possible to record sequential images and obtain information on the late-time temporal evolution of a primary implosion. The addition of explosives containment will further increase the utility of the FXR facility.

The second DARHT axis is scheduled to be completed in the year 2000. With the full dual-axis facility, we will be able to obtain information on implosion symmetry as well as limited temporal information.

The DARHT and upgraded FXR facilities will also provide information useful for the design of an Advanced Hydrotest Facility. Experience gained from double-pulse operation of PHERMEX and FXR and dualaxis operation of DARHT will be essential in optimizing operations and experimental design for this multiple-beam, multiplepulse facility.

- Improved Understanding of Materials Properties. Accurate simulation codes and physics models are required for extrapolations past the "leaping-off point" of hydrodynamic tests and hydronuclear experiments. To make these codes more accurate, we must improve the accuracy and completeness of the material property databases on which they draw. Inadequacies are often related to materials that are difficult to work with (for example, plutonium) or to regimes of pressure and temperature that are difficult to access in the laboratory.

The science-based Stockpile Stewardship Program includes a program of experiments, closely coupled with theoretical modeling, to address the fundamental properties and physical quantities relevant to nuclear weapons. These experiments rely on an extensive set of laboratory capabilities, including the hydrotest facilities described above and a variety of other experimental facilities and measurement techniques, such as:

* High-explosive test facilities.

* Facilities for experiments with plutonium.

* Synchrotron radiation sources, together with diamondanvil cells, for determining material properties.

* Neutron-scattering sources for determining material properties and cross sections.

* Shock tubes and gas guns.

* Apparatus for measuring materials strength at high strain rates.

* Capabilities for processing hazardous and radioactive materials (for example, plutonium).

Typical of the fundamental measurements needed is an improved understanding of the materials science of high explosives and plutonium. This knowledge is needed to accurately predict changes in the primary implosion or in its response to abnormal environments. Experiments to provide such data will include HE detonation studies (for example, understanding the deflagration- to-detonation transition), materials and chemical analysis, and neutron scattering measurements of structure.

A major material property issue is the plutonium equation of state (EOS), which determines the melt temperature of plutonium as a function of pressure. Improving the accuracy of the plutonium EOS will translate into improved predictions of, for example, the heating of plutonium under shock loading. Much of the current data were obtained in high-explosive experiments or with gas guns. Because of the limited nature of data on plutonium melt, active investigations continue using diamond-anvil cells. Other fundamental data, such as the changes in atomic lattice structure as plutonium approaches its melt temperature, are being obtained and will greatly aid the development of plutonium's near-melt EOS.

We also need data on materials properties under dynamic (i.e., nonstatic) conditions. The principal means of obtaining such data has been with explosively driven assemblies-for example, observations of spall or ejecta from shocked materials and measurements of the hydrodynamics of complex assemblies. Gas guns have also been used for ejecta measurements. Laserdriven experiments using minute volumes of sample offer particular advantages for hazardous materials studies.

In addition to the many experiments addressing fundamental materials properties and hydrodynamics that rely on highexplosive drive, some experiments 
are now being conducted using pulsed-power sources. These experiments can complement the HE-driven experiments by providing hydrodynamics studies in different geometries and without complicating features associated with the explosive material itself. Although pulsed-power facilities and the National Ignition Facility are principally focused on high-energy-density issues relevant to secondaries (discussed below), they can also contribute to certain primaryrelated measurements, such as material equations of state. Once ignition is achieved in the National Ignition Facility, experiments on deuterium-tritium gas burn are anticipated; this facility will be the only one available for fundamental experiments involving high-density deuterium-tritium burn, burn propagation, and, potentially, the effects of impurities.

\section{Secondaries}

Even if the primary performs as anticipated, there remain a number of physics issues that affect secondary performance. The performance of the secondary depends on the interaction of many dynamic physics processes, including radiation flow, hydrodynamics and thermodynamics, and fission and fusion neutronics. Equations of state of weapon materials are needed for hydrodynamic modeling, and neutron cross sections of the fissile material and the thermonuclear fuel are needed to predict yield and output.
Insufficient knowledge of basic weapon physics will frustrate our attempts to understand the subtle and complex threedimensional phenomena that will occur as warheads age. Now that nuclear testing has been halted, we must decouple our remaining tools-in particular, computational simulations-from the empirical limitations of the past so that we can answer the technical questions about the stockpile that will arise in the future.

For this discussion, we assume a properly functioning primary as we identify the essential issues for maintaining confidence in the reliability of the secondaries in the enduring stockpile.

\section{Issues of Concern}

To examine the key physical processes relevant to the performance of a secondary, we have broken the problem into four areas: radiation transport, implosion hydrodynamics, ignition, burn, and weapon output and effects.

- Radiation Transport. Our inability to precisely calculate the transfer of energy from the primary to the secondary typifies our lack of complete understanding of nuclear weapon processes. This step in the operation of a nuclear weapon is crucial, since inadequate energy coupling can degrade secondary yield or cause complete failure of the secondary stage. During the nuclear testing years, this lack of complete understanding was not a problem because the radiative energy transfer could be determined experimentally in a nuclear test.

Today, we need improved models of the relevant physics for secondary operation. To perform adequate calculations, we first must describe the time-dependent partitioning of primary energy. We must then accurately model radiation transport from the primary to the secondary. These calculations require, for example, accurate radiation opacities for various materials.

In addition, age-related changes in the primary could cause larger-than-anticipated swings in primary yield. Therefore, we must also calculate the sensitivity of the secondary to primary yield variations.

- Secondary Implosion Physics. Radiation-dominated hydrodynamics plays a major role in determining the implosion symmetry of the secondary. The coupling of hydrodynamics and the relevant nuclear physics. determines the performance of the secondary. Secondary performance is also subject to a variety of hydrodynamic instabilities.

In addition, voids, cracks, and other age-related defects in the secondary's materials could cause complex hydrodynamic anomalies that can cause secondary failure or yield degradation. Voids and cracks are never symmetrical and thus need to be modeled in three dimensions. The required 3D modeling is beyond our current computational capabilities. A new generation of computers and computer codes, calibrated with data from nonnuclear experiments and past nuclear tests, will be needed. 
- Ignition and Burn. Although our calculations are benchmarked against existing test data, energy production in nuclear weapons is not completely understood. We do not fully understand the details of some essential processes and how they interact in ignition and fuel burn. Improved experimental facilities will enable us to study some hydrodynamic aspects of these phenomena. However, many issues related to ignition and burn cannot be addressed in the laboratory.

- Weapon Output and Effects. The output of a nuclear weapon includes neutrons, gamma rays, $x$ rays, explosion-debris kinetic energy, fission products, and activated elements. The ability to calculate the total spectral output of a weapon is an ultimate measure of our understanding of weapon performance. Weapon output is also the basis for describing weapon effects. Many uncertainties remain, particularly the effects of high-energy $x$ rays.

- Effects of Aging on Secondaries and Stewardship of the Aging Stockpile. The crucial performance measure for stockpile stewardship is the ability to know when to decertify a weapon, how to fix the problem that caused decertification, and how to recertify the remanufactured or "fixed" weapon. Without nuclear testing as the ultimate performance demonstration, this capability will be fundamentally different from past practices.
Before we can address the issues of a changing stockpile, we must develop a better understanding of the weapons as they were designed, built, and tested. Production tolerances for the stockpiled nuclear weapons were specified to eliminate $3 \mathrm{D}$ features such as voids. As a result, we have little data on the performance of weapons that do not meet production specifications. As was the case for primaries, our experience with surveillance of stockpile secondaries indicates that aging will introduce $3 \mathrm{D}$ features that may greatly exceed production tolerances and may affect secondary performance. These changes include:

${ }^{*}$ Defects in the radiation case, leading to changes in radiation transport.

* Voids, chips, cracks, or chemical changes in secondary materials, which could degrade the implosion and thereby degrade yield.

Uncertainty about the effects of age-related features stems from our inability to perform highresolution $3 \mathrm{D}$ calculations and

\section{Secondaries}

Key Issues

- Secondary performance will probably deteriorate as aging caivses cradks, voids, corrosion, and material degradation. These problems must be discovered diring surveillance and modeled adequately to determine their effects.

?. Unlike primaries, no aspect of secondary performance can be demonstrated at full scale in the laboratery.

- Secondary performance cannot be modeled adequately with today's codes, each stodk pile weap on tequires empirically adjusted parameters based on nuelear test data.

- Several complementary experimental capabilities will be required to fill gaps in our wherstanding of secondary weapons physics and to validate new codes.

\section{Major Thrusts}

- Define the areas in which secondary perfomance is uncertain. the physical parameters to be explored, and the appropriate experimental techingues to use:

- Destgn and construct experimental facillikes principally based an laser and pulsed power drivers. These factities will allow us to explore the broad tange of conditions that are necessaty to better predict secondary performance.

- Develop computational tods capable of modieling the phy sics and geometry necessary to predict the effects of subtle aging phenomena on secondary performance: 
from the lack of data to compare to the calculations. Calculations based on the types of age-related changes found previously through stockpile surveillance reveal that such features may indeed produce effects that would degrade yield.

At the present time, this is not a significant problem. However, in the future, we may have to address the issue of decertifying a stockpile weapon or weapon system. With few enduring systems, a decertification decision would be significant, and our current uncertainties regarding the degradation of weapon performance with age will be unacceptable. We will need high-resolution 3D simulations, validated by relevant nuclear test and laboratory experiment data, before we can commit to the considerable expense of remanufacturing.

The need to remanufacture stockpile weapons will be a consequence of aging, but the physics issues associated with remanufacturing will not be the same as those associated with aging. As with primaries, environmental and other constraints will likely make it impossible to remanufacture secondaries exactly as they were originally designed. Presumably, the 3D problems that arise because of aging can be minimized during remanufacturing. However, given the likelihood of using substitute materials during remanufacturing, we will need to expand our databases of materials properties (particularly, equations of state and opacities). Moreover, the use of substitute materials during remanufacturing could reduce the value of the historical nuclear test data.

Noninvasive diagnostics for surveillance as well as facilities that simulate, in an accelerated fashion, the aging of the stockpile will be important stewardship capabilities. If potential problems in the stockpile can be discovered ahead of time, we can do the necessary research and development so that we can promptly take care of them when they arise.

Computer facilities clearly are a major component of any stockpile stewardship plan. Assessing the performance of the aging stockpile will require a new generation of computers and weapons codes. The task of designing the stockpile was relatively simple compared with the task of assessing the safety and performance of the stockpile as it ages. The theoretical design calculations that will be required to assess aging effects will be far more extensive than anything that has been attempted in the past. - Certifying Remanufactured Secondaries. If an age-related change or defect is serious enough to require the replacement of secondary components, we must provide assurance that the fixed or "remanufactured" secondary will function properly. Certifying proper function in a remanufactured secondary will rely almost completely on computational simulations. Other than nuclear testing, which is currently banned, there is no way to test secondary operation at full scale. Nonnuclear facilities like the proposed National Ignition Facility and pulsed-power systems can provide valuable information, but we must extrapolate those results to the physics regimes relevant to nuclear weapon secondaries. As with primaries, we need significant additions to our scientific understanding together with an improved predictive capability for modeling secondaries.

\section{The Program for Addressing these Issues}

To address the stockpile stewardship issues regarding secondaries, we require enhanced computational and experimental capabilities.

- Improved Predictive Capability for Secondaries. In the past, nuclear weapons were designed, built, tested, and then certified as to reliability and performance. This process involved intensive calculations but was experimentally driven-that is, the calculations were adjusted to match the data. In the absence of nuclear testing, changes in secondary performance due to aging or remanufacturing will be determined through calculations. This will require an improved understanding of nuclear weapon physics and phenomena.

Problems with stockpile secondaries will, like other weapon components, most likely be caused by aging. Subtle effects, such as cracks, voids, and corrosion, will require complex, 3D theoretical design calculations. However, there are few empirical data for validating these calculations. As noted earlier, we must enhance our calculational models, and validate them with data from past nuclear tests and 
appropriately scaled laboratory experiments, to provide the necessary predictive capability.

Data from Nova experiments have been used to develop a set of algorithms to calculate radiation opacity more correctly and economically. However, Nova data are not sufficiently energetic to deal with all materials and conditions of concern to secondaries. - Improved Experimental Capabilities. The challenge for the Stockpile Stewardship Program is to develop adequate experimental capabilities for validating calculations. In Section II, we described the energy produced in the various phases of a nuclear explosion. High-explosive techniques, in the form of hydrodynamic and hydronuclear experiments, can address the pressure-temperature regime for the prenuclear phase of primaries. However, modeling the post-nuclear, high-energy-density physics regime in secondaries is much more difficult.

Although the energy of the nuclear phases cannot be duplicated in laboratory facilities, the energy densities and material conditions can be approached by heating small masses. This heating can be accomplished with lasers or pulsed-power systems. Lasers produce the highest energy densities, but pulsed power heats larger volumes for longer periods of time. These two complementary approaches can produce high-temperature, high-density plasmas relevant to secondary weapon physics.

- Inertial Confinement Fusion. The national Inertial Confinement Fusion (ICF) program is an outgrowth of a 40-year effort at DOE's national laboratories to understand the physics issues and to develop a routine capability to produce DT ignition and fusion burn on a laboratory scale. To achieve this goal, the ICF program has developed a series of highpowered lasers and ion beams as well as highly sophisticated numerical models to verify ignition conditions.

In the "indirect drive" ICF process, short pulses of beam energy are used to heat assemblies (hohlraums) containing pellets loaded with DT fusion fuel. Physics problems in ICF, such as symmetry of the implosion, radiation opacity, mix, and fusion burn, parallel key issues in secondaries. Synergism between the ICF and weapons programs has contributed directly to improving the predictive capability of each.

The major facility used for laser-driven ICF research is Livermore's Nova laser, a 10-beam, 40-TW glass laser. The largest facility for generating ion beams is Sandia's Particle Beam Fusion Accelerator II (PBFA II), a 50-TW, 12-MV ion accelerator that produces lithium beams for ICF applications.

- Large Glass Lasers. Building on experience with the Nova laser, we have developed a program to investigate physics issues relevant to secondaries. Nova is being used to study radiative opacities, hydrodynamic mix, high-pressure shock phenomena (pressures up to $750 \mathrm{Mbar}$ ), and radiation flow. In a recent laboratory-scaled experiment, Nova was used (by Los Alamos scientists) to simulate the performance of a weapon radiation case; the results of this experiment improved our understanding of radiation hydrodynamics simulations. An advantage of large, multibeam glass lasers is that individual beams of the laser can be used for different purposes. Recent opacity experiments have been remarkably successful, in large part because we could use one of Nova's ten beams to measure with high accuracy the temperature and density of the heated matter and a second beam to generate a source for measuring radiation absorption. This experiment and others have generated valuable and constraining data relevant to secondaries.

- The National Ignition Facility. Weapons physics regimes of interest for many issues of secondary performance require higher material energy densities than can be achieved with Nova. The next-step facility in the ICF program is the 196-beam, 600-TW National Ignition Facility. This facility will establish the feasibility of ICF by achieving ignition, an accomplishment that will open opportunities for the use of fusion for civilian energy production. Moreover, once ICF ignition is demonstrated, this facility can serve as a laboratory testbed for studies of DT ignition. The National Ignition Facility will also greatly improve our ability to access, in a laboratory environment, conditions of temperature, pressure, and energy density approaching those of nuclear weapons.

Calculations for the National Ignition Facility predict that this facility will produce (without 
ignition) radiation environments close to those of interest in weapons. Thermonuclear ignition of the DT fusion fuel with the National Ignition Facility will produce an even closer match to conditions of interest to nuclear weapons. No other facility, existing or proposed, can achieve these conditions. For well-characterized experiments at these high temperatures and pressures, the National Ignition Facility is unmatched for the science-based Stockpile Stewardship Program.

- Pulsed-Power Facilities.

Pulsed-power techniques use electrical energy conversion and storage systems, such as capacitor banks or explosive generators and power-conditioning systems, to tailor the voltage, current, and time scale of an electrical pulse to an experimental assembly. Pulsed power can deposit large amounts of energy into a small volume very rapidly. In one configuration of interest, electromagnetic energy produced by the pulsed current flowing in a thin cylinder of material causes the cylinder to implode along its axis (this is often referred to as a $z$-pinch). Although pulsed-power technology of this type is not as mature as that for laser-produced plasmas, the large amount of energy available from pulsed power makes this technique extremely valuable for addressing design and physics issues associated with both primaries and secondaries.

The pulsed-power-driven implosion produces high-energydensity plasmas, which in turn produce large amounts of radiation. These plasmas are complementary to laser-heated plasmas in terms of their energy, size, and time scale. The total delivered energy, working volume, and time scale that can be produced by pulsed-power facilities are larger than those possible with laser facilities although the pressures and temperatures achieved are lower.

Fast pulsed-power techniques ( $<300 \mathrm{~ns}$ ) can compress and heat plasmas to temperatures of a few hundred electron volts, accelerate ion beams for ICF research, or accelerate electron beams to create radiation sources for weapons effects simulations and weapons physics experiments. Relevant weapon physics areas that can be investigated using fast pulsed power include $x$-ray generation, radiation flow and symmetry, and spectral and temporal tailoring of radiation fields.

Fast pulsed-power facilities include Sandia's Saturn facility, which produces more than $500 \mathrm{~kJ}$ of radiation from a $z$-pinched imploding liner in 15-ns pulse widths. In addition, the PBFA II accelerator could be modified to produce more than $2 \mathrm{MJ}$ of radiation, which would provide intermediate scaling data for future experiments at $15 \mathrm{MJ}$ with Sandia's proposed Jupiter facility. The demonstrated temperature of $65 \mathrm{eV}$ in large $\left(10-\mathrm{cm}^{3}\right)$ vacuum hohlraums and $100 \mathrm{eV}$ in small $\left(0.5-\mathrm{cm}^{3}\right)$ foam-filled hohlraums on Saturn scale calculationally to $175 \mathrm{eV}$ and $240 \mathrm{eV}$, respectively, on Jupiter. Although the Jupiter facility was proposed initially to address weapon effects issues, it may prove to be the best facility for studying radiation flow at moderate temperatures.
Slow pulsed-power systems (300 ns to several microseconds) can accelerate plasma liners to velocities of 3 to $5 \mathrm{~cm} / \mu \mathrm{s}$, producing temperatures on the order of $100 \mathrm{eV}$. This technique promises the largest amount of driver energy offered by any aboveground technology, which will make possible high-energy-density experiments at large (centi-meter) scale. Physics areas relevant to weapons can be investigated using slow pulsed power, including:

* Hydrodynamics (e.g., high symmetry, melting and freezing, movement of joints).

* Hydrodynamic instabilities (e.g., instability growth, material mixing, shear flow).

* Other issues related to heating, large-scale material motion, and radiation flow.

Current laboratory facilities can address second-order implosion hydrodynamics issues in which radiation is not dominant, including the motion of voids or cracks. Capacitor-bank facilities, such as the Pegasus facility at Los Alamos, can deliver 0.5 to $1.0 \mathrm{MJ}$ to an experimental volume, while the proposed Atlas facility will deliver 2 to $4 \mathrm{MJ}$. Pegasus has demonstrated implosions with less than $0.5 \%$ asymmetry, which enables precision hydrodynamic experiments.

Explosive pulsed power has the potential to deliver even larger amounts of energy to a target. The Procyon generator at Los Alamos can deliver 1 to $1.8 \mathrm{MJ}$ to a target, and future explosive pulsed-power systems should be capable of storing $100 \mathrm{MJ}$ and delivering 15 to $20 \mathrm{MJ}$. 


\section{Nonnuclear Components}

T urning a nuclear assembly into a nuclear weapon that meets military requirementsweaponization- requires myriad nonnuclear components to provide: - Initiation functions.

- Surety-that is, safety, security, and use control.

- Interface and support instrumentation.

There are as many as several thousand nonnuclear components in a typical nuclear weapon. The more than 5500 nonnuclear parts for a B61 bomb are shown in Figure IV-1. Because of the demanding safety, reliability, and use-control requirements as well as the constraining weight and size limitations, many of the nonnuclear components are highly specialized and system-specific.

\section{Initiation Functions}

Nonnuclear components must provide four essential initiating functions:
- Confirmation of the weapon's authorized release and trajectory sensing, thereby permitting weapon firing signals to be generated.

- Tritium and deuterium gas injection to enable boosting.

- Detonator initiation.

- Neutron generation and injection.

These functions must occur in a safe, secure, controlled, and reliable manner, under a wide range of conditions. These

\section{Nonnuclear Components}

hej issues

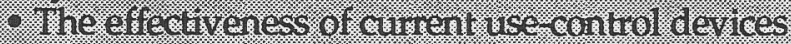
may dearease. With time beraver new methods

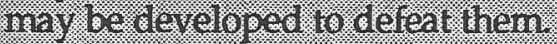

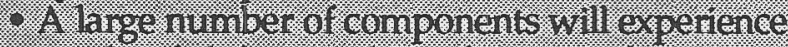

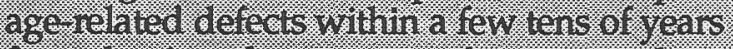

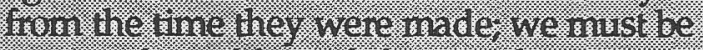

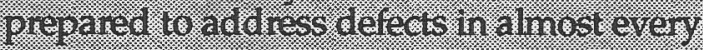

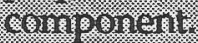

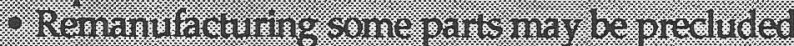

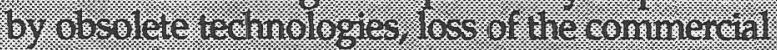

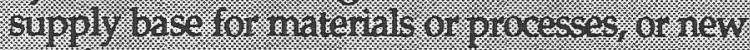

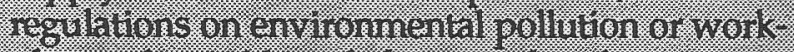

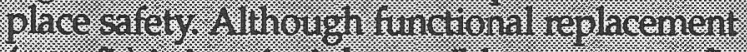

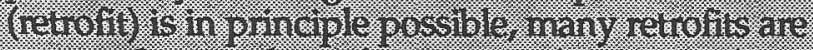

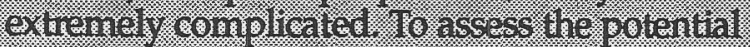

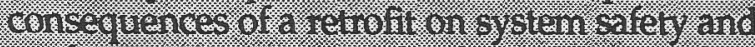

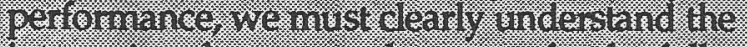

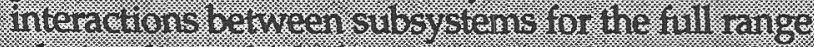

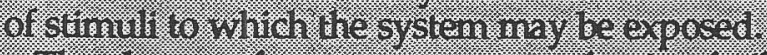

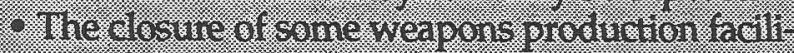

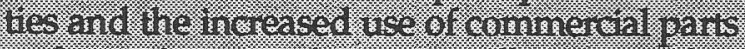

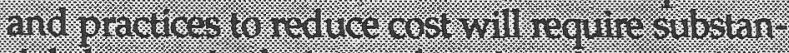

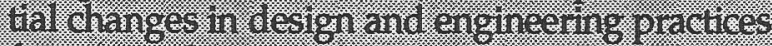

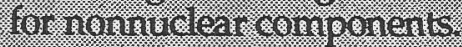

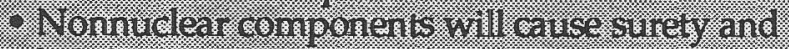

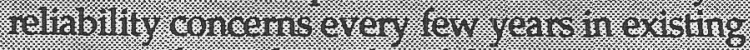

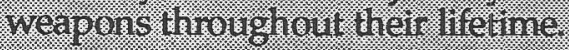

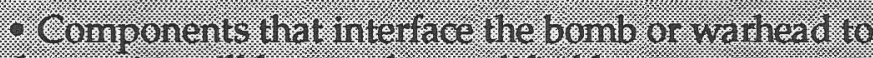

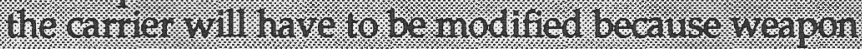

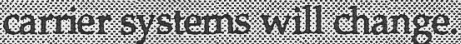

Major minusts

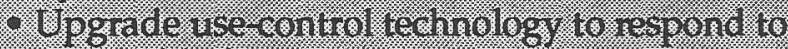

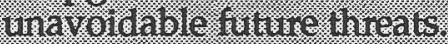

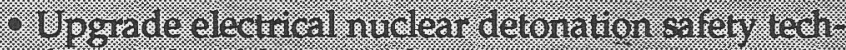

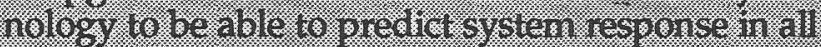
aredible envivin wanents:

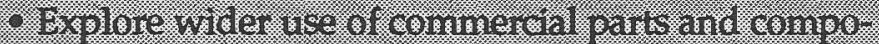

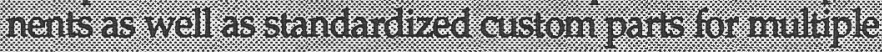
Wearsonis systimas.

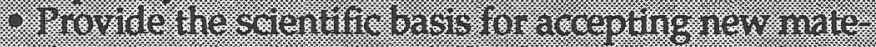

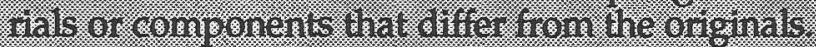

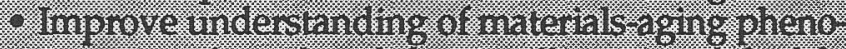

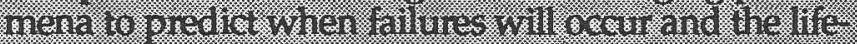

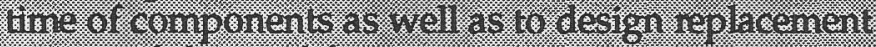

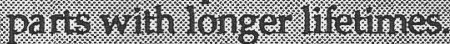

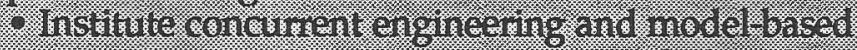

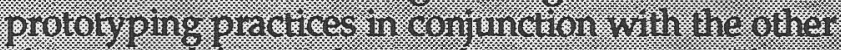

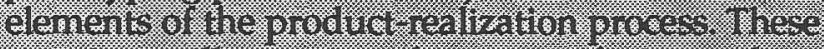

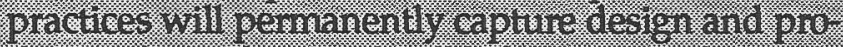

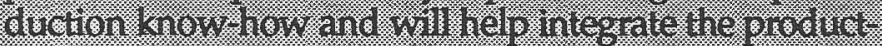

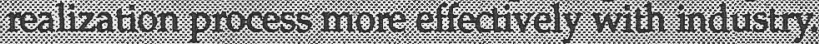

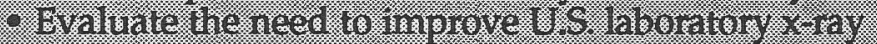

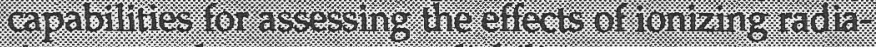
Gron on nuelear weapons re hability. 


\section{Nonnuclear Components}

conditions include exposure to such harsh environments as intense shocks caused by the functioning of other subsystems within the weapon system or by weapon delivery operations (such as impact on hard surfaces) and ionizing radiation caused by a nearby detonation of another nuclear weapon during the time interval between removal from the stockpile and delivery to target.

The major components needed for initiation include:
- Programmers that control the arming and firing sequence.

- Arming subsystems, consisting of thermal batteries, capacitors, switches, and gas-storage and gas-transfer systems for primary boosting.

- Contact, time, and radar fuzing devices.

- Firing subsystems that provide a high-voltage electrical pulse to the main-charge detonators.

- Neutron generators that flood the primary with neutrons at a prescribed time during the implosion phase.

\section{Surety Functions}

Nonnuclear components have essential roles in providing safety, security, and usecontrol (surety). Meeting the requirements for surety is demanding because of the wide variety of conditions for which surety must be assured together with the tight space

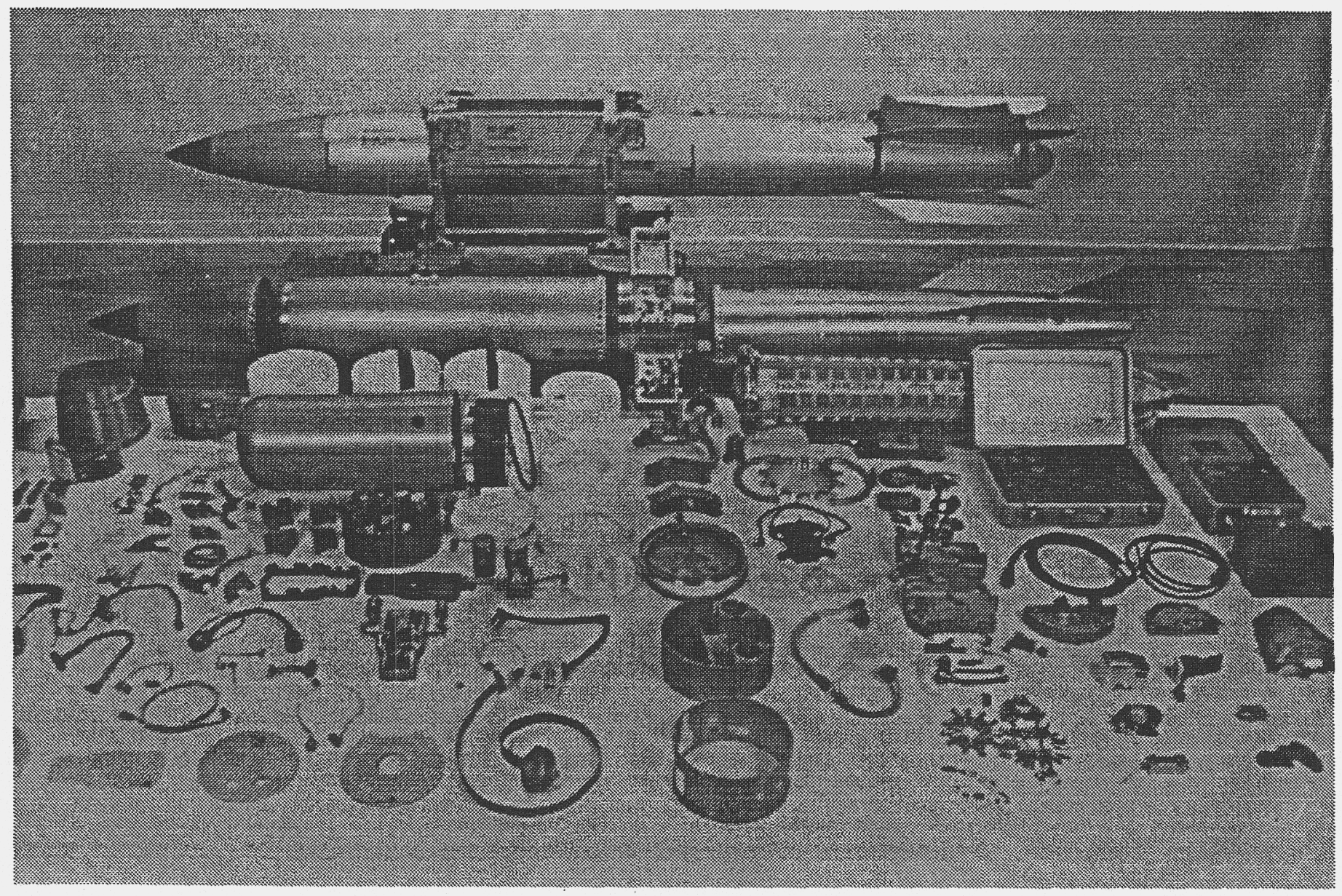

Figure IV-1. There are typically several thousand components in a nuclear weapon, most of them nonnuclear (that is, not part of the nuclear assembly). This exposed view of a B61 bomb shows the more than 5500 nonnuclear parts needed to provide essential functions in a safe, secure, controlled, and reliable manner. 
and weight constraints of a weapon system.

\section{Electrical Nuclear Detonation Safety}

Electrical nuclear detonation safety refers to the prevention of unintentional electrically induced detonation of a nuclear weapon. It is designed into nuclear weapons using data from experiments and predictive models, at both the component and system level. The goal is prevention of unintentional nuclear detonation or dispersal of nuclear materials when a weapon system is subjected to any credible environment. Accomplishing this objective normally involves special design features and requires tradeoffs between performance and cost. Because of the unacceptable consequences of unintentional nuclear detonation or dispersal of nuclear materialls, safety is never compromised for improved performance in system engineering.

Today, electrical nuclear detonation safety is primarily achieved by incorporating electrical exclusion regions that protect the electrical firing system and nuclear system detonators from unintended sources of electrical energy. The intended application of arming energy is controlled by strong-link switches. In addition, some essential firing components are engineered to fail predictably and irreversibly when subjected to specific environmental stimuli within the electrical exclusion region. These devices are called weak links.
Implementation of electrical firing system safety, referred to as enhanced nuclear detonation safety (ENDS), represents today's approach for ensuring nuclear detonation safety. The current standard specifies that the probability of unintentional nuclear detonation be less than one in a billion in normal environments and less than one in a million in an accident or other abnormal environment.

Assessment of nuclear safety is an ongoing and evolving process for all weapons in the stockpile. Although there is a formal process for establishing compliance with minimum nuclear safety requirements during the weapon development phase, additional understanding of nuclear safety threats and weapon responses is pursued throughout the life of the weapon system as new methods become available, as advances are made in computer modeling capabilities, and as knowledge is gained about material responses in severe environments.

\section{Use Control}

Use control is the set of procedures and hardware features that allow authorized use of a weapon yet protect against deliberate unauthorized use by an adversary who has gained access to a nuclear warhead or weapon system. Critical elements of usecontrol technology include coded switches that unlock the weapon and make it ready for use as well as use-denial features that protect against an adversary who intends to cause a nuclear detonation.

\section{Weapon Security}

The Stockpile Management Program is primarily responsible for weapon security, which comprises the positive measures that prevent or delay an adversary from gaining access to a nuclear warhead or weapon system during storage, transportation, and handling. The Stockpile Stewardship Program must provide the technical support required for physically secure stockpile management. Weapon security includes:

- Personnel entry control, detection, assessment, communications, and access delay systems. - Security systems that facilitate the control of nuclear weapons in storage, transportation, and rapid deployment with minimal site preparation and unobtrusive operations.

- Assessment tools to assist in identifying and correcting potential vulnerabilities and to provide cost-effectiveness tradeoff analyses.

- The integration of use control and weapon security.

\section{Interface and Support Instrumentation}

Many nonnuclear components extend beyond the physical limits of the weapons themselves. For example, use-control equipment must be provided to manage release codes and, ultimately, to unlock the weapons for use. Similarly, shipping 
containers and handling equipment must be provided to safely transport and maintain the weapons and their components. Also, many components must be provided to interface the nuclear weapon into the weapon carrier and to provide structural support, environmental protection, and aerodynamic features.

Other items that must be

provided include:

- Parachutes.

- Spin generators.

- Lightning arrestor connectors.

- Timers.

- Detonators.

- Mechanical assemblies.

- Test equipment.

- Handling gear.

- Shipping and storage containers.

- Associated training and maintenance manuals.

\section{A Science-Based Approach to Weapons Engineering}

Science-based models have been a cornerstone of weapon safety and reliability assessments throughout the history of the U.S. nuclear weapons program. There is no room for error in safety engineering of nuclear weapons. The probability of unintended release of nuclear energy or dispersal of nuclear materials when exposed to any credible abnormal environment is certified to be less than one in a million and less than one in a billion per weapon-year for normal environments.

Since it is impossible to physically simulate all of the possible abnormal scenarios to which a weapon may be exposed, extrapolation between experimental tests cannot be avoided. These extrapolations are valid if we have a sound understanding of the weapon system, including accurate and complete representations of the underlying physical and chemical processes.

Because of the severity of the potential physical stimuli (e.g., strong shock waves, high temperatures, pulses of electrical energy, and combinations of such stimuli) and the complexity of U.S. nuclear weapon systems, broadly based scientific understanding coupled with computational simulations have provided the foundation for our system-response predictive capabilities. Computer simulations are much less costly than experiments and field tests. However, to reduce the cost of stockpile stewardship by relying more heavily on computer-based predictions of safety and performance, we must place safety engineering of nuclear weapons on a complete and sound scientific basis.

In the past, evolving military characteristics drove the need for innovative technical solutions for nonnuclear components and system integration. Often, these innovations were made possible only by new scientific understanding derived, in part, by nuclear testing.

Maintaining the safety and reliability of the enduring U.S. stockpile will bring new challenges requiring new scientific insights. Components will fail. Remanufacture to original specifications after extended periods of time will likely be hampered by the obsolescence of certain technologies or the unavailability of special materials.

Consequently, in lieu of redesign, we will have to engineer new materials to exacting specifications or, alternatively, establish a scientific basis for accepting replacement materials or components that differ from the originals. This, in turn, demands expertise and advanced capabilities in materials science and system-level performance modeling.

The smaller, less diverse, older stockpile of the future will be more susceptible to commonmode failure. To minimize the effect of such failures, we need to develop a scientific basis for predicting the impending functional failure of individual components. To support this goal, we will develop first-principles aging models to gain scientific understanding of age-related changes in materials.

Also in the past, dedicated weapon production plants and deliberate capability assurance programs guaranteed that specialized parts could be produced for the lifetime of each weapon type. Most weapon manufacturing processes are empirically established prescriptions, and the processes are controlled by people skilled in a narrow manufacturing art. Like all arts, these specialized skills atrophy without practice.

The cost of maintaining many of these infrequently used but essential production capabilities for the smaller enduring stockpile is clearly prohibitive. Therefore, a fundamental change 
in the product realization process is needed to reduce the cost of maintaining these capabilities. We must also develop ways to ensure the ability to make reliable products in small lot sizes at affordable costs. One area where such a remanufacturing capability is essential is application-specific microelectronics hardened to withstand ionizing radiation.

We will satisfy these requirements, for appropriate processes, by replacing empirically based process prescriptions with model-based process descriptions. Unlike peoplebased skills, computer-based models are timeless. If appropriately implemented, model-controlled manufacturing will permit remanufacture to exacting specifications even after long periods of production dormancy. Virtual prototyping, in which simulations of component performance are combined with modeling of the appropriate manufacturing processes during the design phase, is well suited to replacement weapon components. Virtual prototyping is especially suitable for intermittent, small-lot production of reliable specialty products. Again, scientific understanding of the relevant systems underlies successful development and implementation.

However, the scientific underpinnings of many of the design and manufacturing processes used for weapon components are not well understood. Consequently, to realize the potential of model-based design and production, we must gain new scientific understanding in these systems.

Broad science and technology bases in materials, electronics, and engineering are also needed to devise new weapon components to meet evolving safety and reliability requirements. For example, integrated sensors for monitoring the state of health of individual stockpile weapons (see Section VII) will require breakthroughs in engineered materials, specialized manufacturing capabilities, and in-depth understanding of electronics and photonics.

Clearly, in order to provide weapons engineering support of the enduring stockpile at affordable cost requires continually advancing expertise in a wide spectrum of science and technology-materials, electronics, manufacturing processes, and computational simulations, to name a few. Substantial effort and investment will be required, initially, to institutionalize this fundamentally new way of providing essential engineering support; however, once implemented, this science-based, virtual-prototyping approach to weapons engineering and manufacturing will be the most efficient and cost effective in the long term.

\section{Issues of Concern}

The issues of concern center on how to cost-effectively retain the science and skill bases needed to address inevitable agerelated stockpile problems and new military requirements. On the basis of historical stockpile data, age-related defects are expected to occur throughout the lifetime of the stockpile. We cannot predict the rate at which defects occur or the components that will be affected in the enduring stockpile, and thus we must be prepared to replace every component in a weapon for as long as that weapon remains in the stockpile. As previously discussed, remanufacture or replacement of components will likely be complicated by the loss of dedicated production capability, obsolete technologies, or interrupted external supplies of special materials or components. Furthermore, we must be prepared to meet new military requirements for interfacing equipment to new or modified weapon carriers and for replacing components that are no longer acceptable. For example, use-control technology will need to be upgraded because of external advances in countering technology. The basis for these and other concerns are amplified as follows:

- The enhanced nuclear detonation safety (ENDS) approach to implementing nuclear safety is sound in principle but implementation has not reached fully assured safety. First, it is impossible to define every credible environment that a weapon could be exposed to. Second, it is not possible to simulate some of them. Third, the interdependence between multiple stimuli has been difficult to demonstrate for safety subsystems that rely on weak links and a common exclusion region barrier. Also, margins between exclusion region integrity and weak-link failure 
cannot be demonstrated over the full range of abnormal and connbined abnormal environment scenarios. Less-than-perfect implementations of the ENDS approach are referred to as nuclear safety "soft spots." Much of today's development and stockpile improvement efforts is aimed at improving these weaknesses without significant departures from either the underlying philosophy or the basic firing-system technologies.

Some older weapons in the nuclear stockpile do not fully incorporate ENDS and therefore need to be improved. There are two arguments about incorporating ENDS in older weapons: On the one hand, assuming that all weapons should be made as safe as possible, there is justifiable reason to retrofit these older stockpile weapons with ENDS. On the other hand, most of these older weapons are scheduled for retirement within the next decade. Adding ENDS would divert resources that might better be used to further improve the nuclear safety of the enduring stockpile.

- New stockpile stewardship requirements for fixes to agerelated problems will continue to arise. Past experience indicates that we can expect actionable defects (those that require system modification or improvement) to occur throughout the lifetime of a weapon system. Such defects can result from materials aging (inevitable changes in physcial characteristics that cause failure of proper function) or materials incompatibility phenomena that occur or become evident only after some period of time after entering the stockpile.

- A steady supply of limited-lifetime components (LLCs) will be required. These LLCs must be replaced periodically because of well-known changes in physical properties (e.g., tritium decay in neutron generators). Although LLC requirements will usually be met by remanufacture to original specifications, remanufacture may be precluded at some future time by more restrictive environmental or health regulations or by technologies that have become obsolete. In such cases, retrofits may require extensive development instead of remanufacturing to ensure that there is no compromise in system safety. In other cases, new component designs may be needed to meet new military requirements or to reduce ongoing maintenance costs.

- Public pressure for higher levels of safety may challenge the adequacy of present designs and components.

- In the past, some nuclear weapons were designed to withstand possible threats caused by the effects of nearby nuclear detonations. These systems were "certified" for such extreme environments through a combination of underground experiments that used nuclear weapons to produce the desired threat environment and aboveground facilities to produce intense pulses of neutrons, $x$ rays, or gamma rays. Unlike other certification requirements for nonnuclear components, certifying $x$-ray hardness levels will be difficult without nuclear testing and may require new experimental facilities.
(The programs to meet related future manufacturing and production requirements are discussed in Section VI.)

\section{The Program for Addressing these Issues}

We plan to fundamentally restructure the way we design and procure nonnuclear components. Foremost, we will institute practices to more fully use scientific understanding and sciencebased models in responses to requirements for redesign, remanufacture, retrofit, and upgrade. We will also more fully use external technology and supply bases, with an emphasis on commercial products. To facilitate the move to more extensive outsourcing, we will work closely with industry at each step of the product realization process, with an emphasis on model-based process definition. To reduce internal staff requirements, we will institute agile engineering practices, made possible partly by virtual prototyping whereby the components themselves and the processes for manufacturing them are designed and defined simultaneously with no or minimal physical prototyping and testing.

The specific activities needed to address these key issues fall into seven categories:

- Limited-lifetime components.

- Enhanced nuclear detonation safety.

- Age-related problems.

- Use control.

- Military requirements.

- Manufacturing requirements.

- Weapon-effects certification. 
Limited-Lifetime Components

The Stockpile Stewardship Program must provide designs for modified limited-lifetime components. This must emphasize retrofit LLC designs that offer increased lifetimes and that are not system-specific but can be used for several systems. In some instances, such design changes will require the redesign of other components due to size or space limitations or intercomponent functional synergisms.

\section{Enhanced Nuclear Detonation Safety \\ Our goal for nuclear safety} is to deploy warhead architectures and designs that are absolutely safe in any combination of abnormal environments. Most of the concepts for absolute nuclear detonation safety center around the nuclear device and focus on schemes for predisablement, such as techniques for preventing the criticality of the primary unless deliberate action is taken to make the warhead ready for use. However, absolute safety cannot be achieved unless the hardware for generating the safety-critical weapon system electrical signals also offers predictability comparable to that of the warhead when exposed to any credible abnormal environment.

On the basis of past experience, deficiencies in ENDS technology will become evident as we gain improved understanding of the complex interactions caused by unintended stimuli from accidents or other events. Consequently, continued atten- tion to safety assessments and engineering will be needed for as long as the systems remain in the stockpile or until greatly improved safety concepts are developed and deployed. The goal is to achieve absolute safety in all credible abnormal environments. Exploratory research and development to this end will include:

- Replacing today's electronic firing sets with their optical equivalents.

- Replacing barrier materials and designs with ones that offer predictable response to a wider range of accident scenarios.

- Replacing electrical/mechanical strong-link switches with micromechanical or optical devices.

- Developing detailed models of components and systems to improve predictive capability of their response to severe stimuli. Such models will also be developed to design components that are better able to withstand abnormal environments and delivery modes that are more stressful.

\section{Age-Related Problems}

The program for addressing age-related issues is described in detail in Section VII (p. 55). Basically, it emphasizes gaining an improved understanding of materials aging phenomena through accelerated aging studies, backed by appropriately detailed computer models, and will help identify potential failures before they occur. This understanding will also be used to design retrofits that require less frequent inspection or service.

\section{Use Control}

Upgrades of use-control technology will be made available to stay ahead of advances in the technologies that might be used by an adversary to defeat it. Areas of improvement include denial of access to special nuclear material (SNM), remote recoding capability, and unlocking devices based on target recognition as the unique enabling signal. Enabling technologies for target recognition include recent advances in very-high-frequency monolithic, millimeter-wave integrated circuits and ultraminiature antennas.

\section{Military Requirements}

We must be prepared to meet new military requirements dictated by new missions or new delivery systems. Thus, we must be able to provide modifications of the nonnuclear components that interface bombs and warheads to system carriers.

\section{Manufacturing Requirements}

We anticipate that standard commercial parts and processes will be used much more than they now are. Because effective implementation of standard commercial parts into nuclear weapon components and subsystems will often require substantial nonnuclear-component design changes, the Stockpile Stewardship Program will provide for the necessary new designs and associated testing.

Electronics pervade all nuclear weapon systems in the enduring stockpile. They provide the basis for components 
that, for example, interface the weapon to its carrier and provide use-control, firing, fuzing, and delivery. Many of the current electronic components were obtained through specialized procurements involving technologies that are no longer in production and for which no functional equivalent is currently available commercially. In the future, nuclear weapons will rely even more heavily on microelectronics-based components to accommodate higher levels of monitoring and self-test features embodied in the concept of the self-aware weapon (see Section VII).

Although the majority of the required electronics will be obtained from the private sector, some will not be commercially available. The DOE plans to retain a microelectronics facility capable of producing functional replacements for electronic devices no longer commercially available or otherwise unavailable from the private sector. This facility will also be available for limited manufacturing of prototype or low-volume specialized parts for system upgrades.

\section{Weapon Effects Certification} As a element of stockpile stewardship, we will assess the radiation hardness requirements for the enduring stockpile. The current radiation requirements specified by the DoD have not been changed from those established during the Cold War. Replacement components must be certified to current specifications unless lower levels are authorized by the military services. A reassessment of hardness requirements may lower the hardness levels, but they will not go to zero. Although some parts that currently require hardening may not need it in the future, the majority will have to be certified before they are placed in stockpile systems.

Current radiation certification capabilities may not be sufficient to meet future requirements. A new facility may be needed to provide certification of hardness to $x$ rays at fluence levels that were previously provided only by nuclear tests. To this end, we will perform preliminary research and development to assess the technical feasibility and estimate the cost of the proposed Jupiter facility. 


\section{System Integration}

A broad collection of unique skills and capabilities is needed for weaponization - the process by which the functional requirements for the weapon are converted into integrated system designs and prototype hardware. The system engineers who perform this function must ensure that the nuclear and nonnuclear components of a weapon meet performance requirements, as a system. To carry out this function, the nuclear-assembly engineers and those engineers responsible for the other subsystems and components must work together as a team in consultation with the component design specialists.
Ultimately, the system engineers are responsible for nuclear safety as well as for system reliability.

System integration is extremely demanding because of the range of harsh environments in which nuclear weapons must function reliably and remain safe and secure. Not only must a large number of components individually meet performance requirements but the interactions between components must be clearly defined for the full range of stimuli to which the system may be exposed. These interactions can influence the structural, electrical, and nuclear performance of the weapon subsystems.
Harsh stimuli that must be considered in these studies include strong shocks from detonations of a number of nearby high explosives (including the primary main charge) and a wide spectrum of harsh environments resulting from accidents or other abnormal events.

A combination of expert judgment, complicated experiments, and complex modeling and simulations is required to predict the effects of such interactions on system surety and performance. Since it is impossible to physically simulate all credible environments to which a system may be exposed in an accident,

\section{System Integration}

$\mathrm{rey}$ issues

- Nudear weapon sy siems engineering requires unilg we stalls and engineerning test capalbintties to ensure that these sy stemis remain

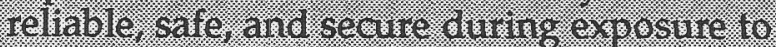
harsh eq Viromments. Inese skills and capabili

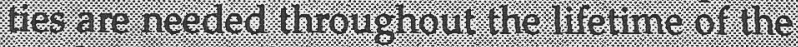

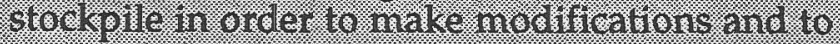

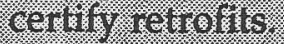

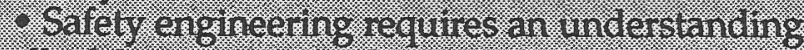

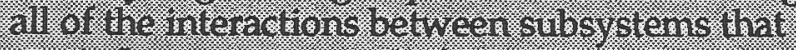

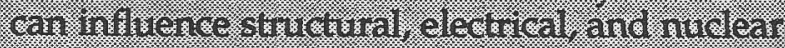

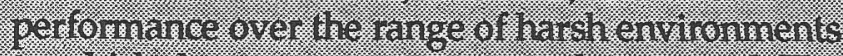

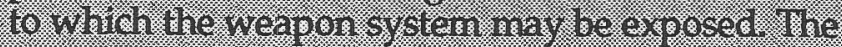
sately eonsiderations of seme of these mieractions

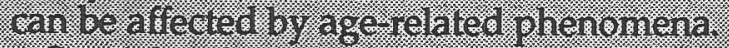

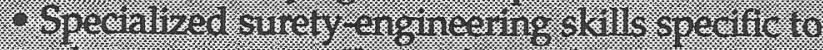

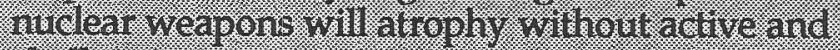
Ärallenging gystent develophent programs.

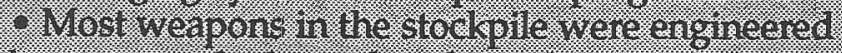

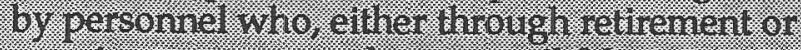
reassigrment, ane no IOnger a valiable.

- The sperinum of testing capalonthes upon whing system ergineers alepend to verify designs and

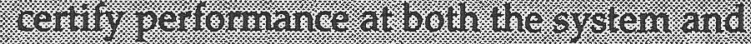
sulosystem level mist be matimtaned at an acreptable cost.

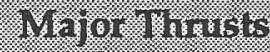

- A d vance the science of high comserguence

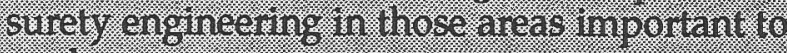

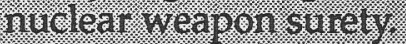

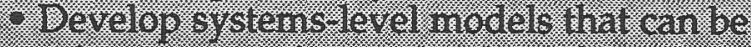

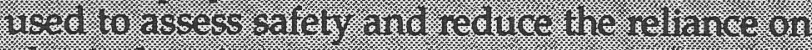

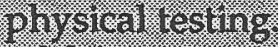

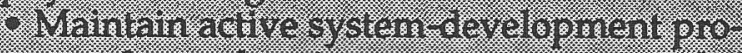

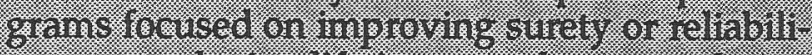

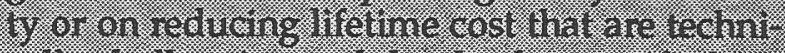

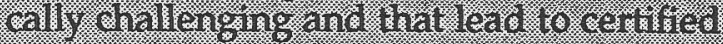

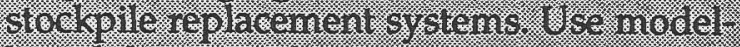

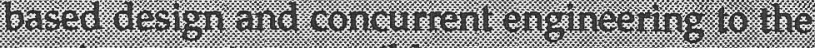

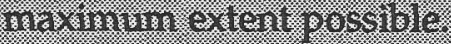

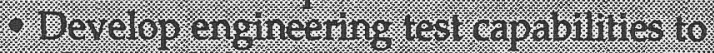

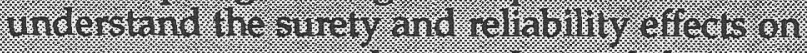

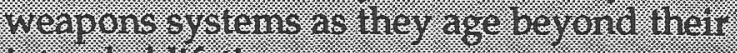

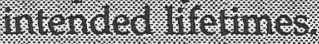

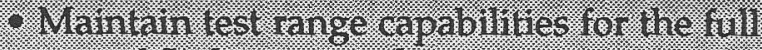
range of finght lests and systems resting. 


\section{Vystem Integration}

other abnormal event, or hostile radiation environment, safety and reliability assessments have relied extensively on scientific understanding and science-based models.

Figures V-1 and V-2 illustrate some of the challenges that nuclear weapon system engineers face when designing reliable systems that will not detonate or disperse nuclear material if exposed to abnormal environments. It is obviously impossible to imagine all credible accident scenarios and to simulate many of the ones thought to be a concern. Although other military systems and some nonmilitary systems face similar concerns, their consequences of system safety are much less severe than for nuclear weapon systems and therefore are less challenging. Figure V-1 shows the damage resulting from an accident that ejected a nuclear warhead from a Titian II silo. Despite the severity of the accident, no nuclear energy was released and no nuclear material was dispersed. Figure V-2 illustrates one of the many challenges faced in designing and engineering systems to operate reliably in the harsh environments of a normal stockpile-to-target sequence; the B61 bomb shown here is designed to operate flawlessly after severe shock resulting from impact with the ground.

The system engineers' responsibilities span all the weapon phases, from design, to certification of a new weapon and its introducton into the stockpile, to final retirement and dismantlement. The last two areas include:

- Helping to assess potential problems that are detected in stockpile systems.
- Devising solutions or repairs to problems.

- Modifying systems to meet new requirements.

- Providing expert advice during dismantlement operations.

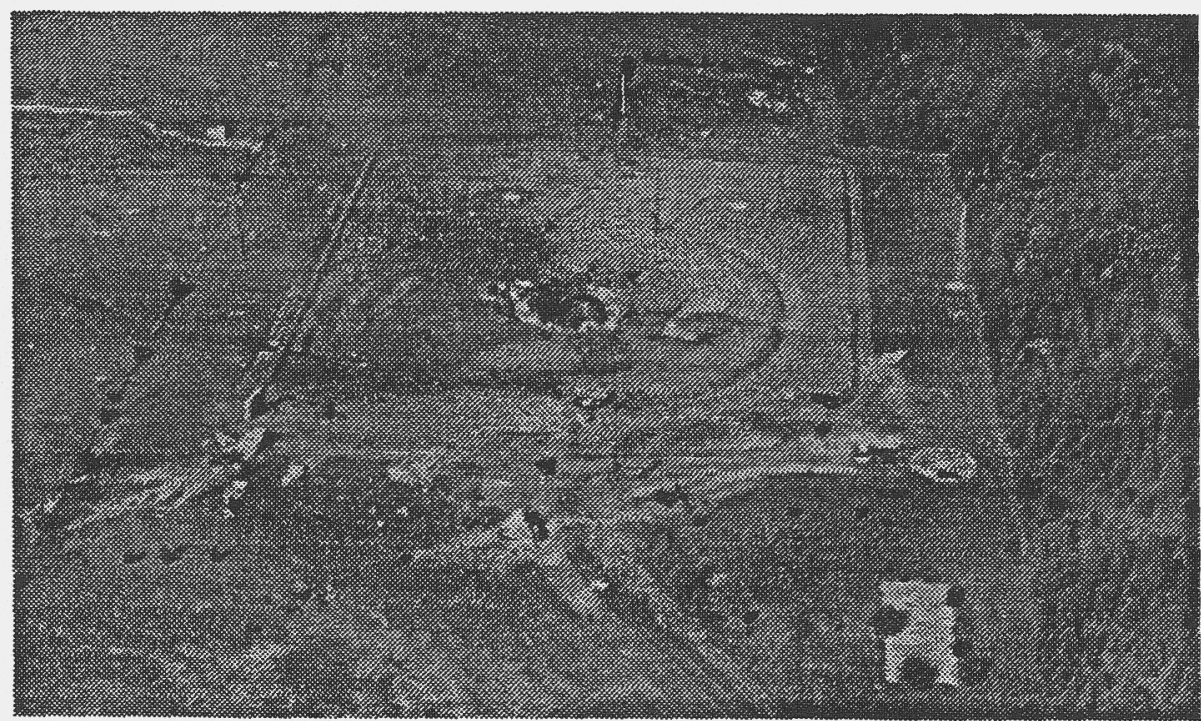

Figure V-1. On September 19, 1980, an accident at a Titan II ICBM site resulted in the explosion of fuel vapors, which ejected a nuclear warhead from the silo. This accident occurred when a repairman dropped a heary wrench socket, which rolled off a work platform, fell into the silo, and struck the missile, causing a leak from the pressurized fuel tank. About eight and a half hours later, fuel vapors within the silo ignited and exploded. The warhead landed several hundred feet from the silo.

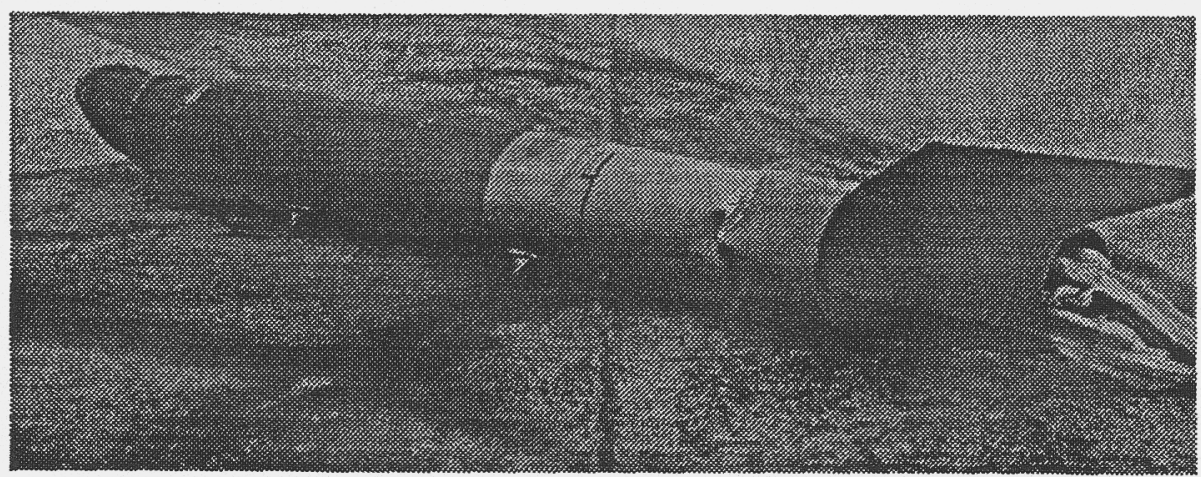

Figure V-2. A B61 joint test assembly after a flight test. The normal impact velocity for the bomb is 80 to 100 feet per second at an angle of 30 to 90 degrees from normal (perpendicular). 
The challenges involved in modifying or improving an existing weapon are often much more difficult than those faced when developing a new system. Design options are limited by constraints imposed by the existing warhead design and by the existing carrier to which it is interfaced. In addition, when making modifications to extend the lifetime of the enduring stockpile, weapons system engineers will face a new set of challenges, such as aging problems that must be addressed without degrading system surety or performance.

\section{Issues of Concern}

The system integration issues of concern are as follows: - The unique aspects of nuclear weapon systems combined with the extremely demanding environments over which these systems must function require a collection of skills and engineering test capabilities that are unavailable elsewhere in the U.S. These skills and capabilities are needed throughout the stockpile's lifetime to design and certify modifications and retrofits and to provide advice and consultation in dismantlement operations.

- Surety engineering requires a clear understanding of all the interactions between subsystems that can influence their structural, electrical, or nuclear performance in the broad range of harsh environments to which the weapon system may be exposed.

- Surety concerns arising from age-related phenomena must be addressed because there is an increasing number of weapons in the stockpile whose lifetimes have been extended longer than originally intended.

- System engineers' specialized skills will atrophy without active and challenging system development activities. If the systemengineering skill base shrinks too much, confidence in nuclear surety assessments and safety engineering will be compromised.

- Many of the people who engineered the nuclear weapons in the stockpile have since retired or been reassigned. Existing documentation for some of these weapons may not be adequate to enable others to rapidly address surety or reliability concerns.

- Many existing environmental test facilities that system engineers depend on to verify designs and certify performance of the nuclear and the nonnuclear subsystems are being closed, raising the specter of compromised solutions to future problems or requirements. Many of the test facilities in jeopardy are unique to the nuclear weapons complex. For example, the Tonopah Test Range is used for flight tests of systems to evaluate the performance of weapons (containing mock nuclear assemblies) integrated to weapon carriers in an operational mode. Several other environmental test facilities are used to assess the affects of acceleration, crashes, fire, shock, lighting strikes, neutrons, gamma rays, and $x$ rays. Some of these tests are used not only initially in the design of new weapon systems but also on a continuing basis to evaluate environmental and aging effects on the surety and performance of stockpile weapons.

\section{The Program for Addressing these Issues}

The Stockpile Stewardship Program must provide demanding active system development activities to hone the skills of system engineers and to maintain our competence in certifying the safety and reliability of nuclear weapon systems. System modification and improvement activities without full system development and certification are not sufficient because such activities do not normally exercise all critical skills. The program must offer system engineering challenges to attract new people to the program and to ensure that unique know-how and understanding is passed on to the next generation of specialists. To be effective in honing skills and attracting the level of expertise demanded by nuclear weapons, such system development efforts must be technically challenging and useful.

We will evaluate the requirements and feasibility of options for replacing nuclear testing as a final system test of environmental effects produced by a nuclear weapon on nuclear performance and safety. More comprehensive system integration tools and performance diagnostics will probably be required to compensate for the absence of nuclear testing.

We will work to acquire and record the specialized 
knowledge possessed by personnel who will soon retire. This information may well be critical in the future for evaluating safety or performance concerns (e.g., operational procedures for dismantlement, problems that may be encountered during dismantlement). This documentation will include, for example, knowledge of problems that arose during design, fabrication, and stockpile life as well as abnormal occurrences or environmental exposure during handling and storage.

The traditional stockpile surveillance programs will be reviewed to ensure adequate staffing and documentation. New testing capabilities will be developed as needed to meet future requirements in surety engineering and safe dismantlement.

We will work to improve our computer models and to better integrate system engineering into other facets of the product-realization process, including component design, testing, and manufacturing. This will enable us to:

- Streamline the system engineering process.

- Reduce the cost of retaining essential elements of nuclear weapon systems engineering and testing.

- Document and archive the specialized know-how of the current staff.

To reduce the cost of assessing weapon surety and reliabili-

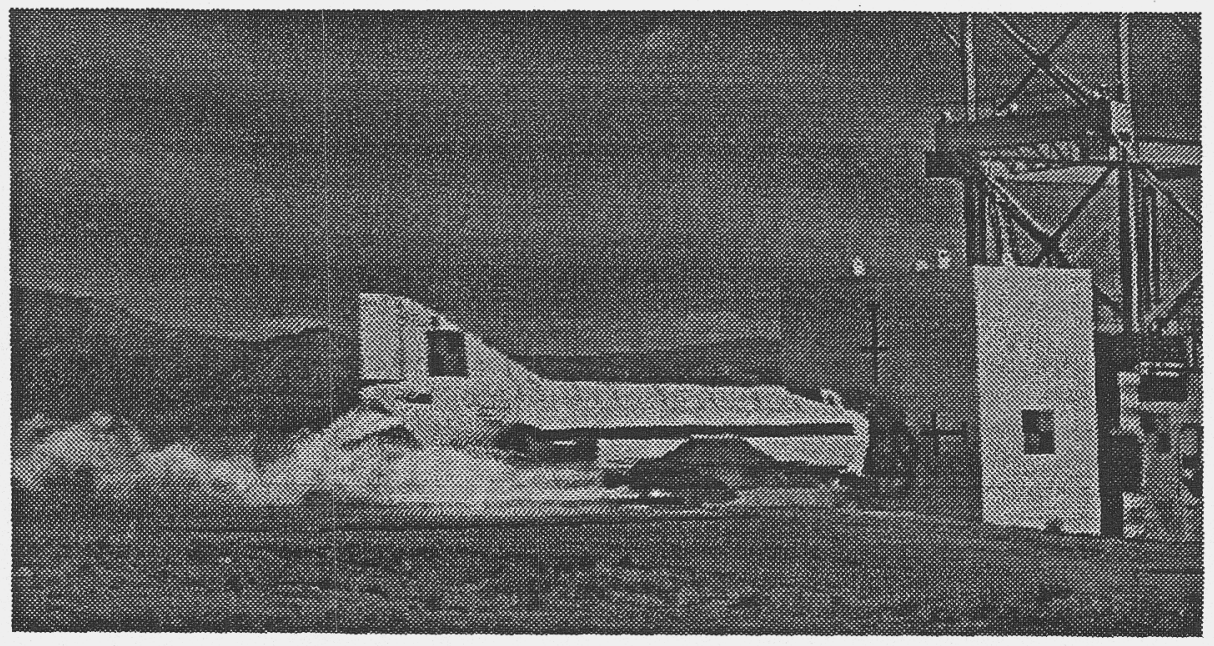

Figure V-3. Test of the impact of a rocket-assisted, full-scale F4 airplane, traveling at 700 feet per second, into a 350-ton concrete block (conducted at Sandia's 3000-foot-long sled track). Although this specific test was not geared to weapon surety assessments, it illustrates one of the challenges faced by nuclear weapon surety engineers. Experiments of this type are extremely costly but are necessary for assessing the saitely of nuclear weapons in abnormal environments. Such problems are beyond the scope of our currentIy computational modeling capabilities; however, selected parts of the impact can be modeled, and simulation results are in good agreement with experimental observations. ty in many complex environments, our long-range plan is to move from a predominately testbased program to one that is based much more heavily on computational modeling. In years past, many experiments were performed and computer models were used to interpolate between tested environments. In the future, we will conduct a smaller number of experiments and will rely instead on the extensive use of models that predict response over an extended range. It will also be possible to perform many safety assessments that cannot be done experimentally because we cannot simulate some credible scenarios. This will require substantial improvements in computer memory and speed and in computational software as well as appropriate and carefully designed experiments to benchmark our model predictions.

Although we have recently made great progress in simulating the types of complex interactions needed to assess safety and reliability concerns, our present modeling capabilities are, in many instances, inadequate for our purposes and some present problems are intractable. The complexity of both the experiments and the models is illustrated in Figures V-3 and V-4. (See Section VIII for a discussion about the computer hardware and computational software needed to address such complex problems.)

We will also:

- Use model-based prototyping and concurrent engineering 

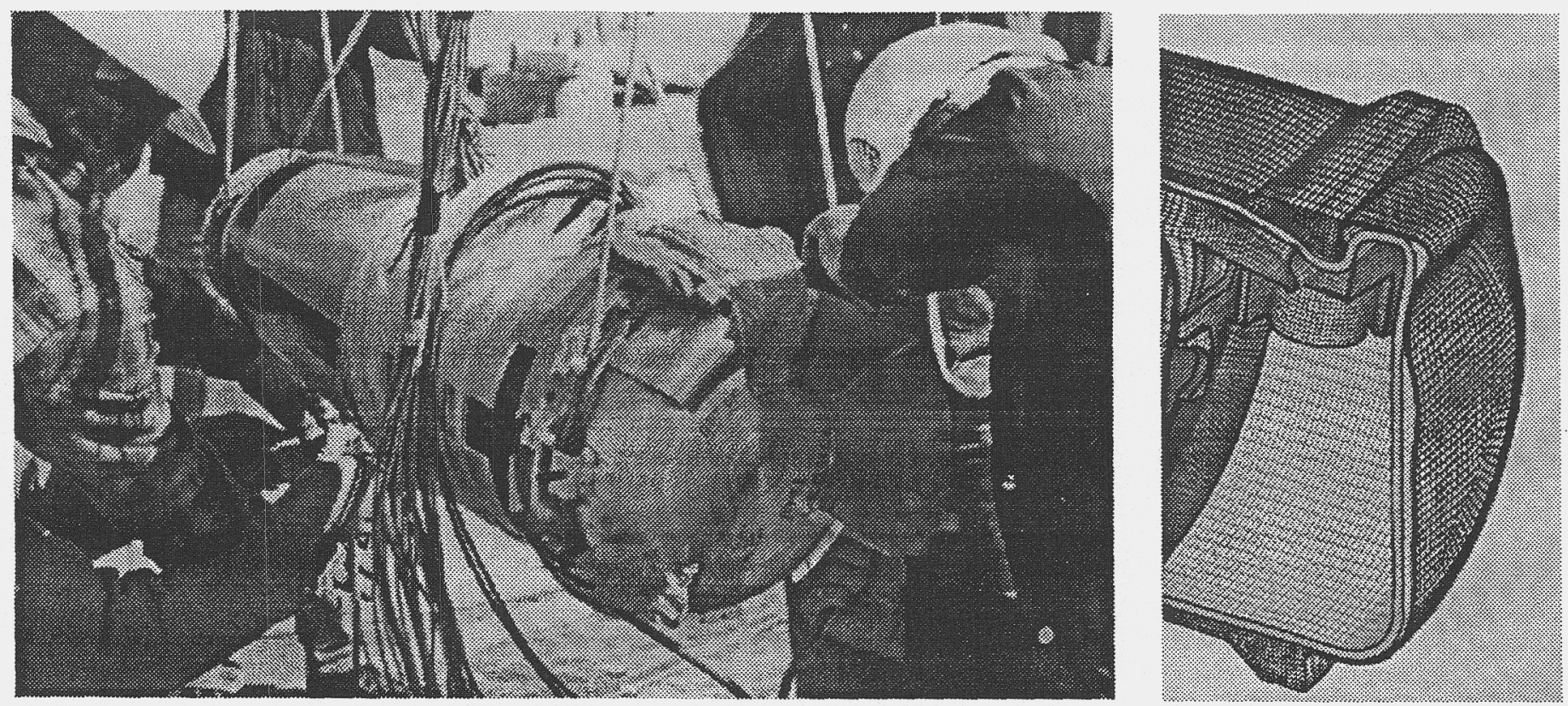

Figure V-4. Structural analysis of nuclear warheads is essential for assessing safety in the event of an accident. Guided by data obtained in carefully designed experiments, computational simulations are used to predict structural response in abnormal environments. The configuration complexity together with the large number of contacting surfaces made up of dissimilar materials pushes current computational capabilities to the limit.

practices, to the maximum extent possible, to reduce the cost of preserving capability, improve intersite and interdisciplinary communications and collaborations, and archive technical know-how.

- Emphasize robust designs for nonnuclear components that offer predictable responses when exposed to all normal and credible abnormal environments.
- Enhance engineering test capabilities to understand the effects on surety and reliability of extended weapon system lifetime in complex harsh environments. - Provide the necessary expertise for devising safe dismantlement procedures and for responding to problems that may arise during dismantlement. This will include deliberate documentation of critical information as well as training programs.

- Enhance the test and demonstration capabilities of the Nevada Test Site, the Tonopah Test Range, and elements of the Nellis Air Force Base to assure a secure remote test complex for systemslevel testing. 


\section{Manufacturing and Materials Technology}

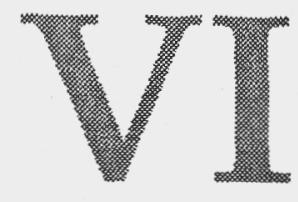

A $s$ noted in previous sections, we must maintain a capability to remanufacture or replace any component in existing weapon systems for as long as these weapons remain in the stockpile. Also, although there is no requirement to provide for new military characteristics of the enduring U.S. stockpile, there will clearly be continuing requirements for improved safety and reliability and for reducing the lifetime cost of the weapon systems. To meet the needs of the enduring stockpille in a cost-effective manner, the product realization process will be fundamentally restructured.

The manufacturing and acquisition of products for nuclear weapons are generally the responsibility of the Stockpile Management Program. However, the Stockpile Stewardship Program is responsible for conducting the research and development for advanced manufacturing, materials, and fabrication technology.

Here we discuss the development of manufacturing processes and the move to new business practices that underpin the manufacturing and procurement activities conducted within the Stockpile Management Program. The requirements for manufacturing and materials that must be met by the Stockpile Stewardship Program are divided, for the sake of convenience, into those for nonnuclear components and those for nuclear components.

\section{Nonnuclear Components}

Currently, the manufacturing process is not highly integrated into the other elements of the product-realization process for nonnuclear components (the process that converts the nonnuclear components, subsystems, and system level requirements into designs and hardware). In the future, the entire
product-realization process must adjust to the requirements of the enduring stockpile. Most important, the manufacturing must be tailored to produce quality parts at an affordable cost in small lot sizes and with intermittent production.

\section{Manufacturing and Materibls Teehmology for Nonnualear Components}

Key. Issues

- Components will have lo be replaced because of their linited Ifretimes, age-relared defects, or new surnely ard rellability requirements. These components nust be replaced at an accept: able cost within a rapidly downsizing production complex. - Intermiltent small-10t production of many different, highty reliable weapon components dinves the requirement for a fundamental restructuring of the product realization process. It must be cost-efficient environmentally bengrn, and responsive to the potential needs of the enduring stockglle for remanutactured. replacement, or new components.

U. U. industry must be nore effectively integrated nto the proalichon proeess:

- Some processes presently used to make materials or parts will becone unacceptable with the introduction of hew enviromment tal and workplace satety regulations.

\section{Major thrusts}

- Create closer ties with U.S. indistry to develop dual-use products and processes (the virtural enterprise) in order to redice the cost or processs development and product prodaction and to facilitate a steady supply base of materials, parts, and components.

- Develop and use virtual prototyping capability (1.e., Complete simulation of the prodict design, perfommance, and manufactur ing processes betore any hardware is produced), and implement concurrent enginieering and agile thanufacturing practices. These practices will be used throughoult the nuclear veapons complex and with approperiate ind ustrial partmers.

- Develop and apply process modblels and sensor based control of processes (smait processes) to ensure affortabble quallty prod: udets in small oot sizes with intermittent production.

- Develop more environmentally benigh and safer processes. 
To cost-effectively acquire quality products in small lot sizes, nuclear weapons manufacturing and procurement must depart radically from past practices. This change must start in the design phase and must wend its way through the entire productrealization cycle. At the same time, the nuclear weapons complex must become smaller, more consolidated, better integrated, and more closely tied to U.S. industry. It must also apply agile manufacturing principles.

To accomplish this objective, products and manufacturing processes will be designed and defined simultaneously, making wide use of predictive models of products and processes. To the extent possible and practical, it will be done by people located at multiple sites working interactively via high-speed communication networks. Throughout the design phase, consideration will be given to environmental and workplace-safety regulations, lifetime system maintainability and maintenance costs, recycling, and final disposition of the product being designed. Major focus will be directed to the development of computer-based process models and the coupling of these models to sensor-controlled feedback to ensure low-volume quality products, even after extended periods of production dormancy.

\section{Issues of Concern}

The need for more cost-effective product realization practices requires much change in the way the DOE has historically made, or

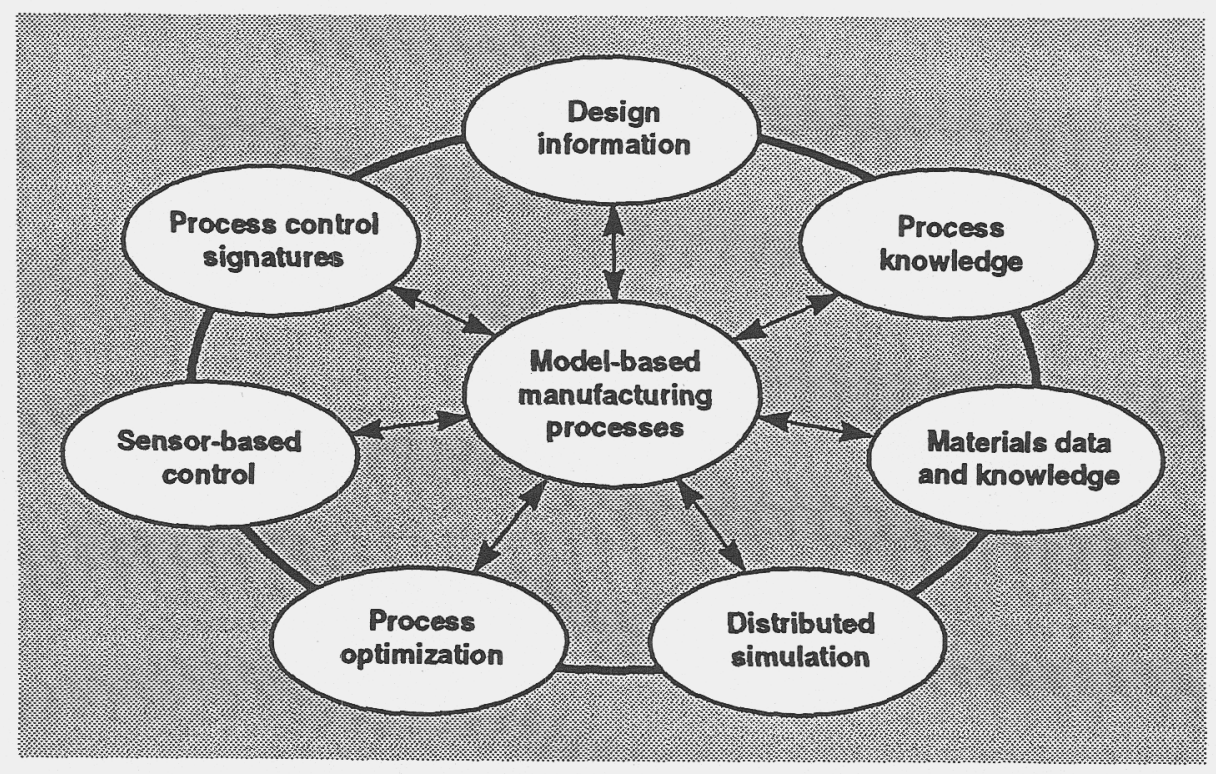

Figure VI-1. The low-volume, low-rate manufacturing that will be required to maintain the enduring stockpile must be based on smart processes that integrate "islands" of specialized knowledge. In addition, sensor- and model-based adaptive process control must be designed into the manufacturing processes.

otherwise procured, weapon materials and components. The dedicated production complex using current practices is too expensive to maintain. To reduce the cost of procurement, we must fundamentally change the way components are designed and produced. The design and production process must be more fully integrated during the design phase, and we must make wider use of industrial technologies, manufacturing practices, and supply bases. As discussed in previous sections, this change will, in turn, require fundamental changes in our internal engineering practices. Changes in manufacturing processes and materials usage will also be driven by environmental and safety issues.

Although the DOE will rely more on industry for the production of replacement or upgraded weapon materials and components, the specialized needs of nuclear weapons require the existence of some internal production capabilities for prototyping products and manufacturing processes.

Internal manufacturing capabilities are also needed to produce specialized parts not available from industry.

\section{The Program for Addressing these Issues}

The DOE has begun an effort to define the "factory of the future," which will be designed to cost-effectively meet procurement requirements in the future. The factory of the future is more a concept than an object. It will be extended beyond the 
boundaries of the DOE facilities and will make possible more widespread and effective use of industrial capabilities. To the extent possible and cost-effective, it will integrate modelbased designs of parts and their manufacturing processes into the product realization process. At each step in the product realization process, due consideration will be given to hazardous waste minimization and potential impact on the environment. Achieving this factory of the future will require a fundamental restructuring of our engineering practices and a fundamental change in the way weapon materials and components are procured.

Focus areas include the following:

- Concurrent Engineering and Agile Manufacturing. Concurrent engineering (integrating design, production, and qualification) and agile manufacturing (making more effective use of equipment and people through time-sharing of expensive equipment and broader responsibilities for the people on the manufacturing floor) will be implemented in the nuclear weapons complex. Industry is deeply involved in developing the underlying capabilities for implementing these practices, and much can be learned from their experience.

The nuclear weapons complex will develop and apply flexible common business systems and a comprehensive communications system between sites. To build in quality and cost control and to reduce the cycle time for new products, process and production engineers will have the tools needed to work interactively with component designers and quality engineers during the design phase. At the same time, a systematic and uniform approach to information flow and communication technology will make more effective use of process capabilities; all sites, including private industry suppliers, will have ready access to manufacturing capabilities at other sites. In this way, the same production capabilities can provide prototype, development, flight test, and stockpile components.
The specialized requirements of nuclear weapons, together with the speed with which these practices need to be introduced into the weapons complex, require an up-front investment and a long-term commitment by the Stockpile Stewardship Program. However, if these practices are effectively implemented, integrated cost savings will soon exceed the initial investment.

- Flexible Workforce. To adjust to a smaller and less diverse manufacturing capability, the smaller weapons complex workforce will be more broadly trained to perform multiple

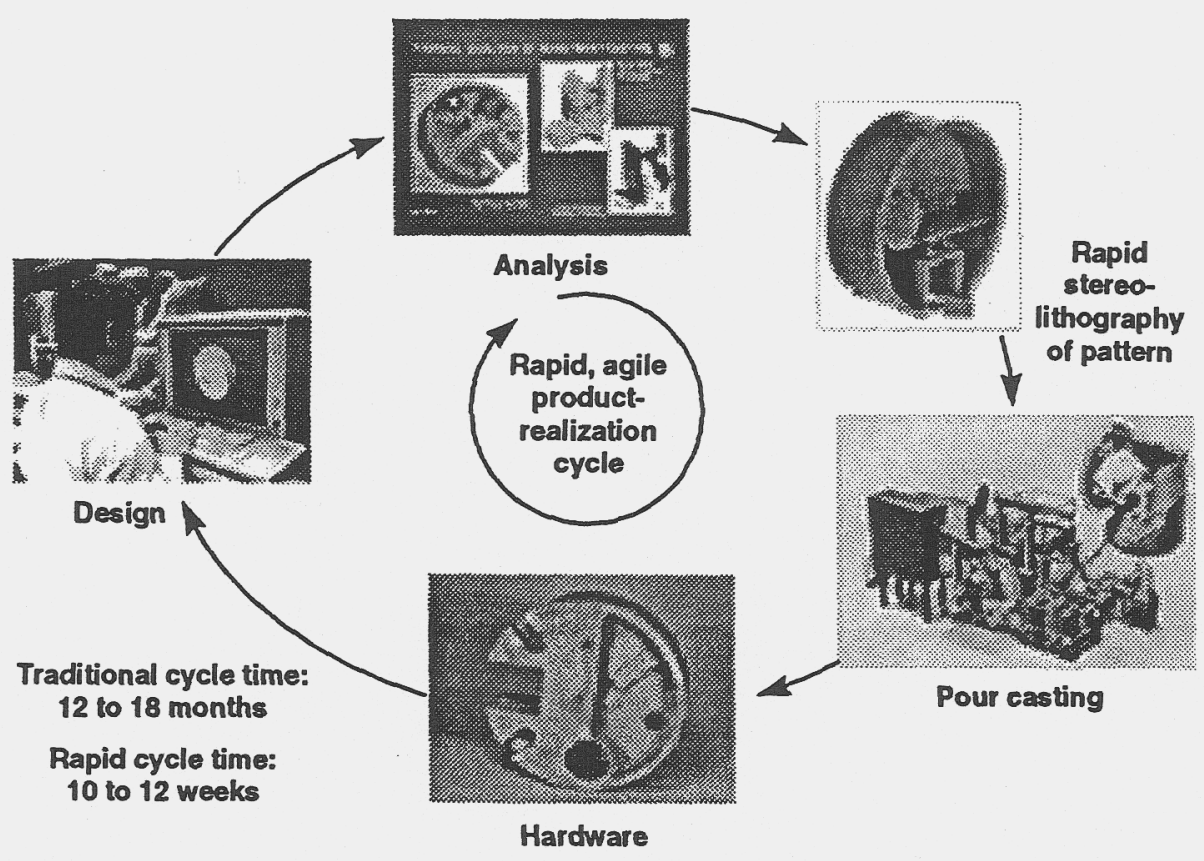

Figure VI-2. Smart processing is already being used for investment casting. A solid model of the cast is developed on a computer using extensive databases of materials properties and computer simulations of the pouring and cooling processes. Improved experimental data are obtained as necessary to define key parameters and interpret observations. The time required to produce the piece shown here was reduced from 12 to 18 months to 10 to 12 weeks. 
tasks and to adjust to the rapidly changing requirements for nonnuclear components.

- Smart Processes. The weapons complex will have an extensive model-based understanding of both nonnuclear component design and the processes used to produce those components. The long-term goal is to develop "smart" processes that merge:

* Computer-generated solid models of the products.

* Electronic information about materials.
* Predictive computer models of the manufacturing processes.

* Sensor-based adaptive control of manufacturing.

The smart-process concept is illustrated in Figures VI-1 and VI-2. Current focus areas for the development of smart processes by the weapons laboratories include investment casting, welding, materials forming, chemical vapor deposition, and plasma cleaning, etching and spraying.

Science- and sensor-based "smart" processes are well suited for the future nuclear weapons complex because they can help reduce the product-realization cycle time and ensure the retention of manufacturing know-how during periods of manufacturing dormancy. With this understanding and capability, the productrealization workforce can use a common collection of flexible process equipment to produce a wide variety of components. Such an approach greatly expands the breadth of the workforce and allows the integration of design and production using concurrent

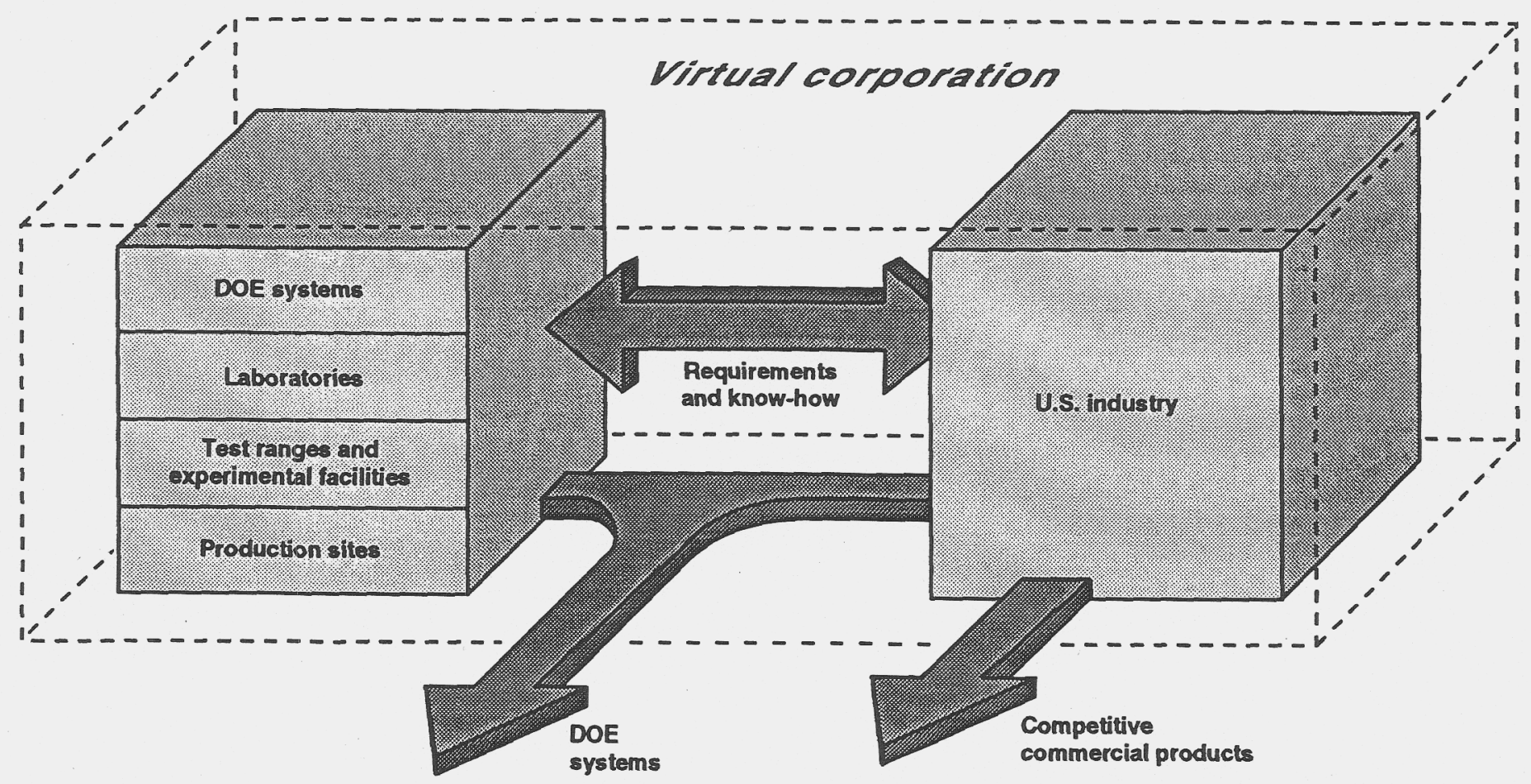

Figure VI-3. To meet the nonnuclear manufacturing needs of the future, the nuclear weapons complex must integrate its expertise with that of U.S. industry to create a "virtual corporation." Except for parts and processes unique to nuclear weapons, which must be maintained by the weapons complex, standard commercial products and processes will be used. The goal of the virtual corporation is to provide demand-driven manufacturing using the most advanced tools of flexible manufacturing and science-based models for process definition and control. 
engineering and demand-driven manufacturing.

Smart processes are also a central element of concurrent engineering and are a cornerstone of the virtual corporation of the future that will link the nuclear weapons complex together with U.S. industry to effect cost-savings and provide effective management of the stockpile (Figure VI-3).

- Low-Volume Production. In the past, the relatively high production volume, the high performance and reliability requirements, and the uniqueness of nuclear weapon functions did not necessarily encourage the use of standard commercial parts or manufacturing processes. Today, we must adjust to a new environment of low-volume production but one that still requires high reliability and unique functions.

Making this adjustment will involve working interactively with industry to develop appropriate products and processes, especially those that have potential commercial applications. Most important, we will form partnerships with industry throughout the productrealization cycle, with a view toward both weapon and commercial applications. Such partnerships may, in many instances, be essential for gaining industrial interest in supplying lowvolume weapons parts. The nuclear weapons complex will retain production of only those products that have no commer- cial potential, demand special security considerations, or must be made in-house to ensure a stable supply source.

- Virtual Prototyping. Eventually, the new manufacturing enterprise will, for some products and processes, use virtual prototyping in which the product design, performance, and manufacturing processes are completely simulated before any hardware is produced. Virtual prototyping of nuclear weapons will require systems-level models that accurately predict performance, reliability, and safety in all credible abnormal environments.

The key to this approach is the concurrent design of the product and the processes used to build the product. This approach will reduce costs by ensuring that required processes and equipment are available and by exploring options during the design phase. The design information can then be fed, in electronic format, to the manufacturing floor where the processes are controlled by computers. This concept is extremely well suited to stockpile stewardship with its need to infrequently build high-quality, highly specialized products.

- Partnerships with Industry. Clearly, the nuclear weapons complex and U.S. industry have many common goals, but each also brings unique expertise to the partnership. Cost-reduction practices and concepts developed by industry, such as concurrent engineering and on-demand manufacturing, will be tailored to meet the needs of the nuclear weapons complex. The multiprogram weapons laboratories have a unique collection of scientific competencies, experimental facilities, and modeling and experimental capabilities. Partnerships between the laboratories and U.S. industry will take advantage of these complementary capabilities. In so doing, the cost of product development and procurement for nuclear weapons will be significantly reduced, and industry will benefit from the development of new commercial products and manufacturing processes.

\section{Nuclear Components}

The principal challenge associated with nuclear-related materials and manufacturing is one central to stockpile assurance-the ability to fabricate, remanufacture, and assemble components of nuclear primaries and secondaries. The ability to fabricate primaries and secondaries is necessary to address problems that occur in aging weapons and to replace or repair them as needed. Manufacturing capabilities are also needed to support any future mandates to incorporate safety improvements that would involve modification of these nuclear components (should such a safety improvement be considered sufficiently important and consistent with our ability to certify the safety and performance of the modified weapon). Although 
some elements of our approach to nuclear-related component manufacturing are shared with those of nonnuclear components, the inability to routinely test the nuclear subassemblies to validate quality creates an essential difference in the approach we must take.

As a result of environmental, safety, and cost issues, many of the production lines and processes originally used to manufacture nuclear components are no longer available. Therefore, we must develop and implement some new technologies. Remanufacturing components that exactly duplicate those of the original weapon, while possible in theory, is not achievable in practice (see "Remanufacturing and Replic-

\section{Manufacturing and Materials Technology for Nuclear Components}

Key. Issuies

- Ability to fabricate, manutacture, repair, and assemble primaries and secondaries is required to ensure stockpile confidence.

- Vany of the production lines and processes originally used to rabricate nuelear components are no longer available and camnot be directly replicated because of new environmental, safety and health standards and downsizing.

- Tritum is required for continued stockpile assurance

Major Thrusts

- Enable a capabillty to fabricate dismantle, and process nuclear-related material components using techmologres that minimize waste and environmental hazandis, mprove worker and public safety and are cost effective and rellible for small 40 t fabrication:

- Preserve the capability to support future decisions to incorporate surety. improvements in stodgpile nuclear. weapons:

- Provide the scientific basis for accepting replacement components that differ from the original comporents.

- Develop enabling technologies for the envirommentally sound disposition of nuclear-related materials.

- Improve the understanding of materials properties and tabri: cation processes as they relate to remanufacturng weapon comn ponents.

- Preserve the knowledge base or the technologies and processes for nuclear-related materials for the US. Stock kpile Maintenance Progran and for reconstitution of production capacity should it ever be needed.

ability," p. 6). We must, however, assure that any changes in remanufactured components do not compromise the weapon's performance or safety.

In addition, to ensure the proper performance of stockpile weapons, we will eventually need a new source for tritium (although this requirement has been mitigated by stockpile drawdown). Tritium decays with a 12-year half-life. The reduced stockpile has also eliminated the need to produce new uranium and plutonium. Emphasis is now on managing uranium and plutonium supplies through storage, disposal, or recycling.

\section{Key Issues and the Program for Addressing Them Regarding manufacturing} and materials technology for nuclear components, we must: - Ensure a capability to fabricate, dismantle, and process nuclearmaterial-related components using technologies that minimize waste and environmental hazards, improve worker and public safety, and are costeffective and reliable for smalllot fabrication.

- Develop technologies for the environmentally sound disposition of nuclear-related materials, particularly plutonium, uranium, and high explosives.

- Support evaluation and ultimate implementation of technologies for tritium production and for extending the supply of tritium.

- Develop an improved understanding of materials properties 
and fabrication processes as they relate to the prediction and evaluation weapon aging and to the remanufacture of weapon components.

- Provide stewardship of the technologies and processes for nuclear-related materials that form the science-based underpinning of stockpille maintenance activities and the basis for reconstitution of production capacity (should it be needed in the future).

The challenges associated with nuclear-related materials and processing are challenges of understanding, technology, and the cost of facility operations under increasingly stringent environmental regulations. Although the major operations are a stockpile maintenance function or a responsibility of the Nuclear Materials Disposition Office, much of the enabling research and development is part of the sciencebased Stockpile Stewardship Program.

- Issues in Remanufacturing and Recertification. It is quite possible that in the future, observed or anticipated changes in the aging stockpile will require the fabrication of replacement nuclear components to maintain acceptable performance and safety. Remanufacturing "to specification" will be ineffectiveand perhaps even detrimentalwithout a process of recertification. Recertification, based on detailed tracking of the remanufacturing process together with appropriate experimental and computational tools and evaluated with expert judgment, is essential to provide confidence that the remanufactured component or weapon will perform as specified. The need for judgment and recertification in remanufacture is related to several factors. Remanufacturing each time we observe a variance from the original specifications is expensive and may introduce unnecessary risk. Disassembling a weapon to replace a nuclear component can be done but is costly and involves nonroutine steps requiring quality assurance. When a variance is found, we must decide whether to leave the weapon alone or whether to subject the aging nuclear "patient" to surgery, recognizing that all surgery runs the risk of unintended detrimental effects. To accurately evaluate whether the observed changes warrant the risks and costs associated with remanufacturing requires expert judgment and the predictive capability developed through science-based stewardship.

Manufacturing specifications are never all-inclusive and some rest in the details of practices that are difficult to document. Major industries, such as aerospace, have recognized the cost and difficulty of trying to remanufacture old products and the associated need to "capture" the production practices. For many complex products, there can be welldefined procedures and specifications, but more subtle variables of process can also have significant effects on product character. All such subtleties would have to be captured to reproduce the product without a change in quality.

We cannot specify beforehand all the measurements and practices that distinguish acceptable from unacceptable remanufactured components. Even with a complete predictive capability, it would be difficult to adequately specify all possible variables ahead of time for technicians to interpret. Is this scratch acceptable? Is that weld a problem? Assurance that remanufactured components are acceptable requires intelligent judgment. Assurance simply by rote examination against a checklist is not a "quality" process.

We cannot realistically ensure the exact duplication of all production processes and practices. Production plants have been closed because it is too costly to maintain a Cold-War-scale operation for today's greatly reduced production needs. In addition, many of the production lines for nuclear-related components have been disassembled, and many of the processes used in the past are now unacceptable for environmental, safety, and health reasons. As a result, in many cases, exact replication of components simply is not possible.

We cannot fully retest the subsystem after remanufacture. Nuclear testing provided the only full feedback and qualityassurance mechanism for nuclear devices. Therefore, the capabilities, understanding, and judgment provided by this 
science-based stewardship program must be developed to provide alternate means for adequate assurance of quality - that is, the means for recertification.

- Plutonium and Uranium. Stewardship of the nuclear stockpile requires the capability to understand, fabricate, process, and dismantle components involving plutonium, uranium, and uranium alloys. This capability is in transition (e.g., with the closing of the Rocky Flats Plant). There are major technical challenges involved in providing this capability while making the transition to achieve cost-effectiveness and reliability for small-lot production, to minimize waste and environmental hazards, and to improve safety. Simple downsizing cannot meet these goals.

Age-related changes in weapon materials can have important effects on the performance of the nuclear components and therefore on stockpile reliability and safety. Given the current nuclear test moratorium and the possibility of a comprehensive test ban, we need an improved understanding of the chemistry, metallurgy, and properties of weapon materials. (Aging is discussed further in Section III.)

As with nonnuclear components, new manufacturing approaches, including concurrent engineering and design as well as agile manufacturing, are required. New manufacturing and materials processing technologies will be based on a detailed scientific understanding of the materials and processes of manufacturing, on the application of new analytical tools (e.g., active sampling and analysis of weld gases for feedback on weld quality, computational simulations of microscopic processes in metal removal to optimize machine tooling design), and on the use of integrated process control sensors in the manufacturing process. Because of the dependence of weapon function on the properties of manufactured components, research and development on fabrication technologies and processes must directly involve weapon designers.

Remanufacturing and nuclear-component production needs for the stockpile will be characterized by small-lot production, potentially with long periods of relatively low activity. This in itself raises issues that require a new technical approach. Without the continuous feedback associated with ongoing production and nuclear tests of early-production units, both materials and production methods must be evaluated to assure that components and assemblies can be certified against appropriate baselines. Expert judgment in weapons design and materials science is crucial.

Small-lot, low-rate fabrication of nuclear components will also require the replacement of the customized hand tooling used in the past with agile manufacturing and inspection technologies. However, some of these processes (e.g., smart welding processes) will be specific to nuclear components. In order to maintain high quality at low cost, the concurrent engineering and design capability will be integrated with flexible manufacturing technol- ogy via electronic information systems and integrated sensors for monitoring and real-time feedback.

Small-lot manufacturing processes also require new approaches to quality assurance and production confidence. For nuclear components, information from advanced surveillance and nondestructive evaluation technologies (Section VII) will provide new measures that can be incorporated in the manufacturing and inspection processes.

New fabrication technologies are being developed to reduce waste streams and other environmental hazards and to reduce worker radiation exposure. Bringing these technologies to fruition over the next five to ten years will require a detailed understanding of these processes as they apply to specific materials. For example, net-shape or nearnet-shape casting and dry machining in an inert atmosphere would minimize plutonium contamination of oil wastes from remanufacturing and other component fabrication processes. They would also eliminate several other of the waste-producing steps that were used to fabricate plutonium parts at the (nowclosed) Rocky Flats Plant. Similar technologies will be developed to minimize wastes from fabricating components from uranium alloys and to eliminate up to one-third of the processing steps. In addition, the Montreal Accords to reduce the use of ozone-destroying CFCs (chlorofluorocarbons) will require new processes for cleaning weapons components. 
- Explosives. Energetic materials explosives, propellants, and pyrotechnics -are key weapon components. Because of the special weapon-related requirements for safety, performance, and precision, many of these energetic materials, or the standards for their formulation and fabrication, differ from those produced for other applications. Existing facilities have adequate capacity for the long-term production and fabrication of these materials. There are, however, three technical challenges to be addressed within sciencebased stewardship: aging, replacement, and disposition and recycling.

Energetic materials are, by their very nature, metastable. Over time, chemical and structural changes can occur in high explosives that may affect primary performance and safety (e.g., by affecting behavior in fires). Over the next decade, we will develop an improved understanding of the fundamental structural, chemical, and physical processes involved in the function and aging of energetic materials. We will also improve our computational models so that these aging-related issues can be better evaluated. The ability to accurately model all phases of explosive initiation, burn propagation, and interaction with other components is needed to certify the behavior of weapons - with new or aged explosives-in their design and abnormal environments. (Research and development associated with these issues is also discussed in Section III.)
Should the high explosive for a stockpiled weapon need to be replaced, we will have to have a scientific basis for acceptance of the new material. We will devise methodologies to ensure that small changes in material composition or necessary changes in process technologies for manufacturing explosive components do not cause unacceptable changes in the material's properties. However, the fabrication, bonding, and assembly of explosive components around warhead pits involves several complicated steps and will still require evaluation and certification. We also anticipate that some ingredients of current explosive formulations may not be available a decade or two from now as industrial technology changes. Substitute materials will be required, and these may deviate sufficiently in some properties to require an assessment of the weapon's safety and performance. Explosives tests, hydrodynamic tests, and hydronuclear experiments (if allowed) will provide ongoing reference data to compare with benchmarks and to support certification.

Thousands of pounds of energetic materials will be removed from dismantled weapons. Reuse of these materials presents a significant research challenge. The only disposal methods currently used are open burning, incineration, and open detonation. We are developing new, environmentally benign chemical processes for disposing of explosives that result in little or no hazardous waste streams.
The goal of these efforts is develop an integrated life-cycle approach that minimizes process waste, recycles or reuses excess materials, and recovers materials for reuse when economically and environmentally advantageous.

- Tritium. Tritium is not now being produced in the U.S. Current tritium supplies will be adequate for several years as the stockpile is reduced. However, ultimately (the date depends on details of the stockpile), we will have to produce additional tritium to replace that lost to radioactive decay.

The principal strategic goal related to tritium is providing a cost-effective and appropriate long-term production source. (The construction and operation of this source would be performed under the Stockpile Management Program.)

There are also issues related to devising the most appropriate method of long-term storage of tritium from stockpile returns and to developing low-pressure, solid-storage tritium reservoirs for improved safety and other benefits to the enduring stockpile. With a smaller stockpile, the yearly need for tritium production will be considerably smaller than the production supplied by the Savannah River Plant's $K$ reactor during the Cold War. Although detailed tritium requirements have not yet been finalized, it is unlikely that $K$ reactor (now in cold shutdown) will be the most cost-effective solution. Other options include some reactor approaches as well as production by means of 
accelerator-based neutron sources. Reactors are generally acknowledged to be more appropriate for larger production requirements. Accelerator-based sources could be more advantageous for smaller inventories.

The best approach will depend on timelines, cost profiles, stockpile and inventory size, and technical feasibility. Necessary enabling research and technology assessments will be important in the next few years. For accelerator-based production, the Los Alamos Neutron Scattering Center (LANSCE) would be uniquely capable of evaluating specific target concepts at high power. The decision to pursue one of these production alternatives will, in the longer term, require development, demonstration, and construction as part of stockpile management. - Other Weapon Materials. There are a number of other materials used in weapon primaries and secondaries that have been selected for their special properties and are critical to weapon function. Many of these materials share issues with the discussion of plutonium and uranium-for example, smalllot fabrication, quality assurance, and assurance of no significant changes in properties of remanufactured componentsand therefore will require similar solutions.

We will develop better recycling processes for these materials. We anticipate that, in the future, some materials or fabrication capabilities will become unavailable from domestic commercial sources due to changing environmental and safety regulations. The manufacturing capability for these materials may have to be retained in the nuclear weapons complex if commercially available substitute materials cannot be sufficiently validated under a comprehensive test ban. 


\section{Stockpile Surveillance}

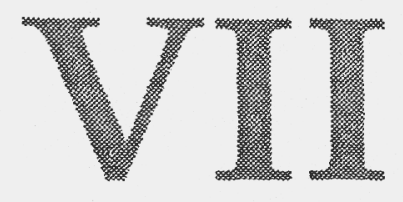

$\mathbf{N}$ uclear weapons in the stockpile are kept in a controlled environment, whether they are on alert or in reserve, for most of their operating lives. To the extent practical, representatives of each weapon type are periodically removed from the stockpile so that they can be inspected and evaluated for possible degradation and so that some nonnuclear components can be tested individually to determine if they conform to performance specifications. Unlike other systems of comparable complexity, nuclear weapons have rarely been operated or tested as a system and will not be now that there is a moratorium on nuclear testing.

Weapon reliability has been assessed primarily through a planned surveillance program that removed a limited but statistically valid number of warheads from the stockpile each year. These warheads were subjected to a variety of tests to assess performance against design standards. (In addition, various components have been saved from weapon production and stored for testing years later to determine the component's reliability.)

In general, there have been three kinds of tests:

- Laboratory tests, which check condition and performance of warhead components against design requirements. In these tests, a weapon is disassembled and evaluated to verify that its subassemblies and, ultimately, its individual components perform to within design specifications. The weapon components selected during production are tested in a similar manner.

- Joint DOE/DoD flight tests, which test the DoD interface to the warhead and verify that the nonnuclear systems function in a delivery environment. In flight tests, the nuclear assembly is

\section{Stockpile Surveillance}

Key issues

- Age-related defects are expected to occur within a lew tens ot years in all weapon types and in all weapon stbsystems:

- Present surveillance methods may be inadequate in the future because they rely on the ability to perform nuclear tests to resolve questions about age-related degradation of the nuclear assembly; in addition, present methods were developed for a larger, more diverse stockpile with a shorter lifetime than the enduring stockpile:

- Currently only a limited number of weapons are evaluated. and problems are usually identified by a functional tailure, providing no advance notice to the component engineers who must devise solutions:

Major Thrusts

- Move from preventative maintenance and periodic inspection schedilles to integrated self-diagriostics for maintenance on demand with the least possible human involvement in order to reduce costs and secuirity risks.

- Retain parts trom older disassembled weapons for study of age-related changes.

4 Integrate state-0) heallih, real-time monitoring systems into every weapon to record the environment conditions to which the weapons have been expesed. These monitoring systems will aid in assessing future changes in rumetionality or materials proper. ties and in looking for symptoms of materials degradation or functional changes.

- Develop neutron and X-ray. Imaging techniques that can image a nuclear system's internal struchure for assessments of the cont sequences of age-related defects in nuclear assemblies.

- Integrate ad vanced telemetry packages into weapons to permit high-fidelity flight tests that require no changes to the system bey ond replacement of the nudlear naterial.

- Pertorm hydrodynamic tests and other experiments to verift implosion characteristics of primaries and to compare these to benchmark results in order to assess the effect of possible agerelated changes on weapon performance or safety.

- Develop an improved understanding of materials aging and compatibility: 
removed and replaced with a mock unit and telemetry system instrumentation. In these tests, the signals to and within the warhead are monitored and transmitted by telemetry to ground-based receivers while the weapon undergoes typical flight and delivery. - Underground nuclear tests, which verify the performance of the nuclear system. With the moratorium on nuclear testing, a combination of aboveground experiments and complex computer simulations will be used to verify nuclear system performance (see Sections III and VIII).

The responsibility for stockpile surveillance is shared by two programs, Stockpile Stewardship and Stockpile Management. The two programs are integrated and complementary. The Stockpile Stewardship Program is largely responsible for forward-looking research aimed at developing better ways to perform surveillance. It is also the sponsor of some of the routine surveillance activities at the laboratories. The Stockpile Management Program is responsible for most of the routine surveillance work at the laboratories and all of the surveillance activities carried out by the rest of the nuclear weapons complex, primarily at the Pantex Plant near Amarillo, Texas. This report covers only those activities included in the Stockpile Stewardship Program.

\section{Issues of Concern}

A surveillance program is essential to weapon reliability and confidence-more than $80 \%$ of the problems discovered in the stockpile have been detected through routine stockpile surveillance. Routine surveillance involves removing a few warheads from the stockpile each year for detailed tests and evaluations; however, this approach has shortcomings. Current surveillance methods were developed when nuclear tests could be performed if a question arose about age-related degradation. These methods were also developed for a larger, more diverse, and younger stockpile than we can expect in the future.

The surveillance issues of concern are as follows:

- On the basis of our stockpile experience, defects are expected to occur within a few tens of years in all weapon types and in all subsystems. As weapons age, agerelated defects, including those due to long-term exposure to radiation, are expected to increase. Most defects have been found in new designs soon after they enter the stockpile but problems have been identified throughout the stockpile life of weapon systems, usually as a result of improved knowledge and understanding. - Only a limited of number weapons are evaluated, and problems are usually identified by a functional failure, providing no advance notice to the component engineers who must devise solutions. (Accelerated aging tests provide advance warning of some impending failures.) - Disassembly methods of surveillance (i.e., "cut-and-look" methods) are costly and require destructive tests of limited stocks of reserve parts.

- The future stockpile will contain older weapons (weapon lifetimes may be extended to 60 years or more) as well as fewer weapons of fewer types. Problems due to aging are often subtle and, with fewer designs in the stockpile, common-mode failures could affect a large fraction of the stockpile. Such common-mode failures are particularly worrisome because a number of the weapon types in the stockpile use many identical components or components with common design features and were manufactured using similar processes.

\section{The Program for Addressing these Issues}

To address the concerns listed above, we will develop improvements in surveillance technology in the following areas:

- State-of-health monitoring.

- Materials aging and compatibility.

- Noninvasive imaging.

- High-fidelity flight tests.

- Stockpile hydrodynamic tests and hydronuclear experiments (if authorized by the President).

\section{State-of-Health Monitoring}

We will explore the concept of a "self-aware" weapon using an integrated network of micro "smart sensors" and "smart selftest features" in the weapon to:

- Monitor the environment (e.g., temperature, moisture, vibration, and acceleration).

- "Sniff" for decomposition products from explosives and plastics. 
- Detect corrosion.

- Check cable continuity.

- Determine the functionality of weapon subsystems.

Smart sensors and self-test features will be composed of sensing and measuring devices with built-in expert systems that can include decision making, data processing, conflict resolution, communications, and distribution of information. For nuclear weapon surveillance, these sensors and self-test features could provide a local, simple status indication; they could also compact and encrypt source data for satellite transmission to a central location. Compacting and encrypting data would provide a real-time monitoring capability and would reduce the dayto-day cost of monitoring the sensors because fewer people would be needed to perform the task. The cost/benefit ratio of central monitoring will, of course, determine whether this approach is indeed practical.

Figure VII-1 shows some potential applications of smart sensors for nuclear weapon surveillance; there are many other possibilities. Although smartsensor technology is in its infancy, it is maturing at a pace that will allow near-term applications for stockpile monitoring.
Based on microsensors using integrated-circuit technology, smart sensors are adaptable to a wide spectrum of chemical species and environmental stimuli. The microsensors can be integrated with advanced digital processors to reduce the datatransmission rate needed for monitoring status to levels that are easily met using today's technology and at modest cost. Coupling smart sensors to optical-data buses is feasible today, and a design based on optical distribution of the information to a nearby radiofrequency transmitter to a satellite link could yield a system that would meet nuclear

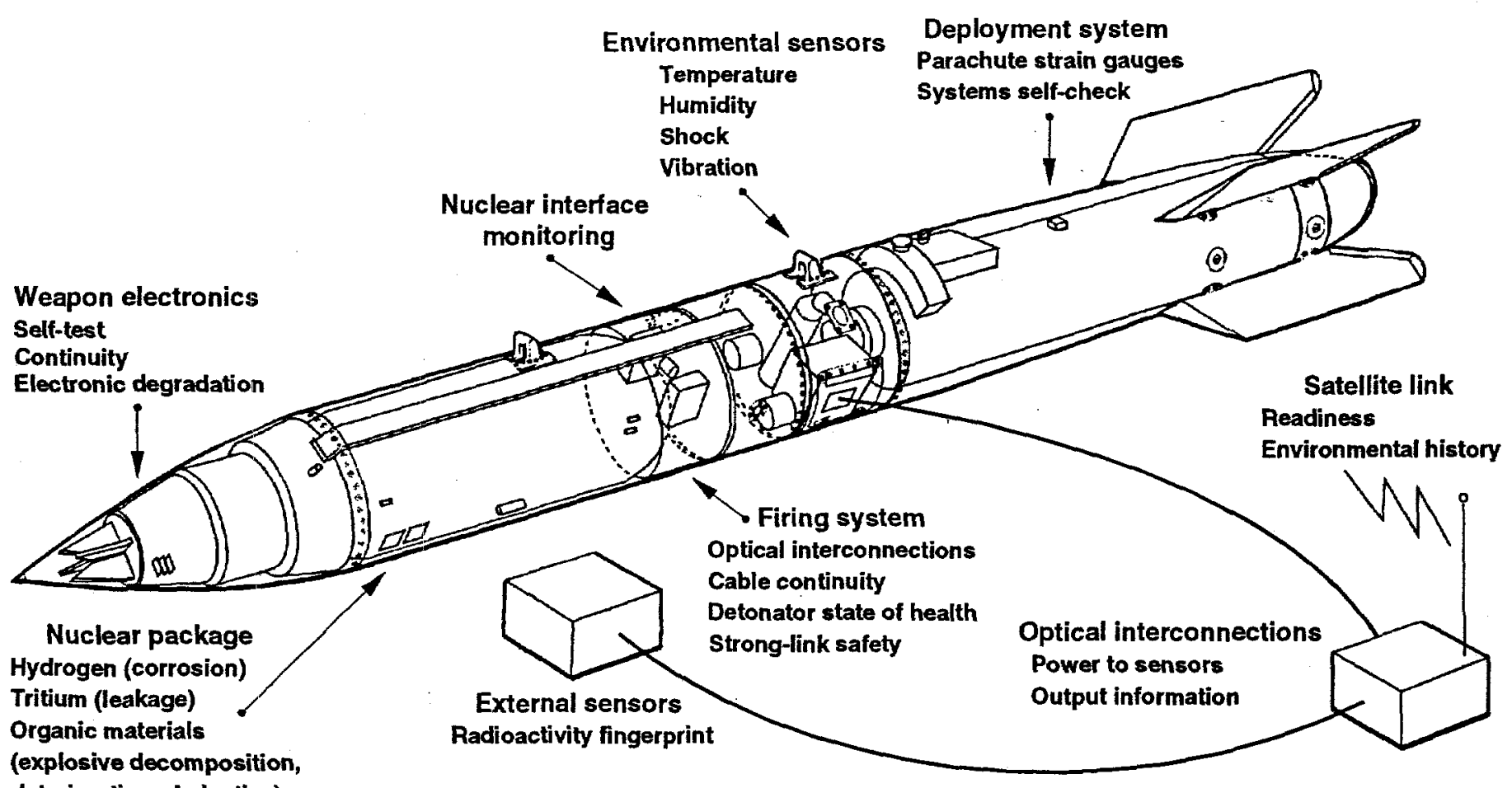

deterioration of plastics)

Figure VII-1. "Smart" sensors in a "self-aware" weapon will be able to predict problems or failure before a malfunction occurs, reducing the need for routine inspection and preventive maintenance and permitting the shift to maintenance on demand. 


\section{Stockpile Surveillance}

safety and use control requirements. High-energy rechargeable batteries to power the integrated sensors are currently available, which would ensure lowmaintenance requirements.

Smart-sensor technology is beginning to find its way into commercial products. (Every late-model automobile, for example, contains some microsensors to alert the driver to possible problems and to assist auto mechanics in diagnosing problems.) Commercial interest in the technology should encourage co-development and dual-use opportunities in, for example, sensor design and manufacturing processes.

Some smart sensors are available now for use in stockpile weapons, including:

- Hydrogen sensors, with a sensitivity as low as one part per million hydrogen in atmospheric air. A photograph of a fieldable hydrogen sensor is shown in Figure VII-2.

- Temperature, pressure, and acceleration sensors.

- A crude spectrometer for photon energies between $10 \mathrm{eV}$ and $10 \mathrm{MeV}$ based on radiation fieldeffect transistors (RADFETs). (A RADFET is based on a radiationfield-effect transistor and provides a total dose measurement in real time.)

State-of-health monitoring could be added incrementally to weapons currently in the stockpile. A partial system could be added during limited-lifetime component changeout, and the full system could be added during a major weapon upgrade.
As promising as state-ofhealth monitoring may be, a number of important technical issues must be addressed before we can even assess the potential benefit and cost of implementation. The sensors, computations, and communication technologies required to fully implement state-of-health monitoring are not mature. System integration and validation concerns must also be addressed. In addition, significant reliability engineering and testing will be required to ensure that the new technologies function effectively without degrading system reliability or surety.

\section{Materials Aging and Compatibility Studies}

We will improve our understanding of materials aging phenomena, including the effects of dissimilar materials in contact with each other, to help extend the lifetime of stockpiled weapons. Many materials used in weapons were selected at a

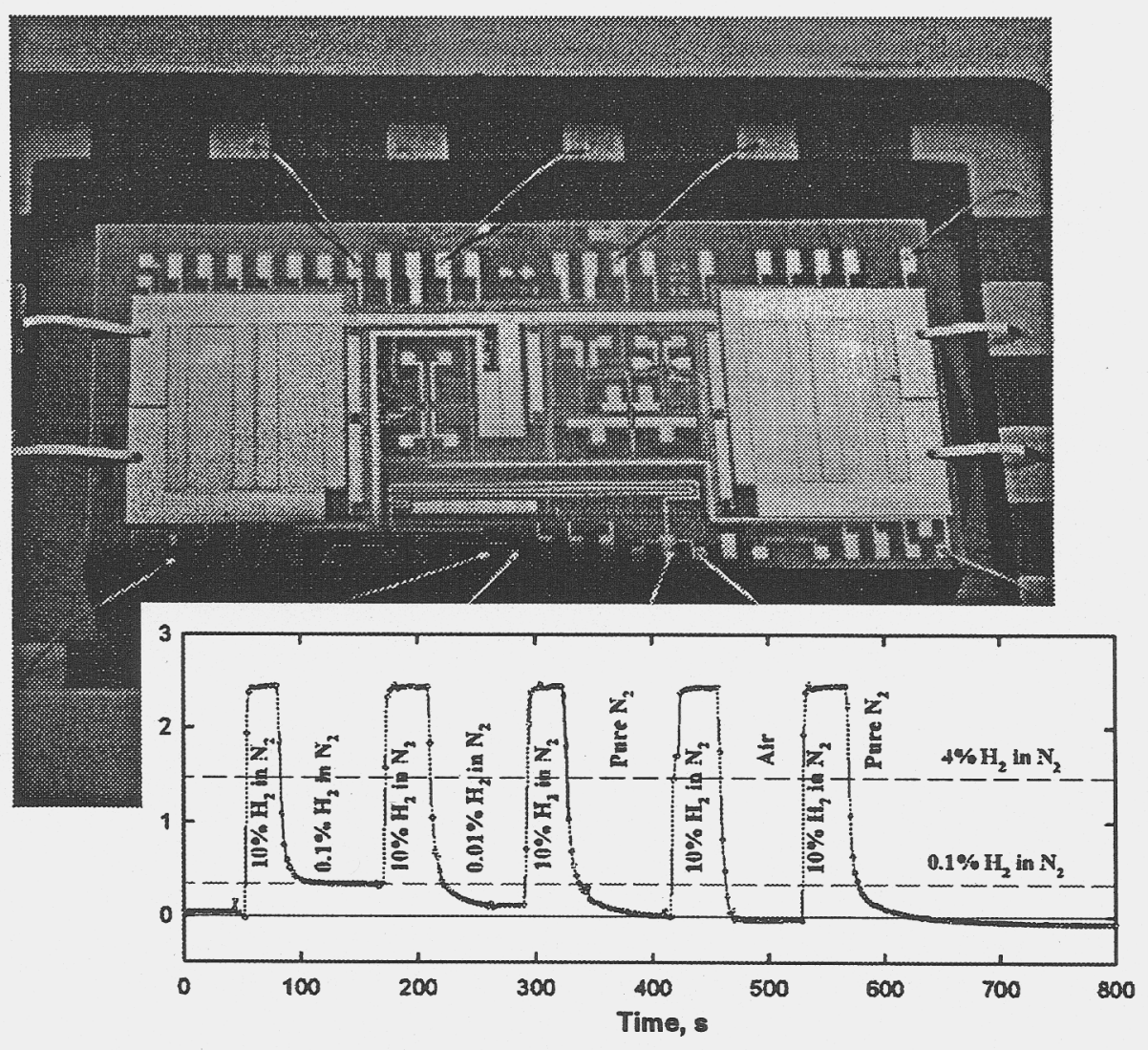

Figure VII-2. Several smart sensors are available now for use in stockpile weapons. This hydrogen sensor measures about $1 \mathrm{~cm}$ square and can detect and measure concentrations as low as one part per million hydrogen in atmospheric air. 
time when the useful lifetime of a weapon was not expected to exceed 20 to 30 years. Some of the materials in the enduring stockpile may not meet requirements, but we do not have sufficient information to assess the issues. We are examining dismantled weapons to improve our understanding of the effects of material aging.

Recent advances in experimental tools, in surface chemistry understanding, and in computer simulations of aging phenomena could lead to answers to many major materials-aging concerns. In addition, these advances could lead to an earlier identification of problems that may affect performance or safe- ty. Appropriate experimental tools currently exist at the laboratories to carry out these studies, but some may need enhancement. Selected experimental capabilities that could be applied to materials aging include:

- Scanning tunneling microscopy to study corrosion phenomena.

- Atomic force microscopy to study the incompatibility of materials in contact with each other.

- Scanning Rutherford backscattering to assess the effects of tritium decay on materials properties and welds.

- Neutron scattering to assess stress buildup in low-atomicweight materials, the formation of cracks, and materials incompatibility.

- X-ray microscopy and radiography to study changes in physical properties of materials. - Intense, narrow bandwidth, tunable radiation from infrared to soft-x-ray wavelengths to study chemical changes caused by ionizing and bond-breaking radiation.

\section{Noninvasive Imaging}

High-resolution radio-

graphs using $x$ rays are already used for noninvasive examination of nuclear assemblies to detect defects in high-atomic-weight materials; however, this technique has difficulty addressing certain issues.

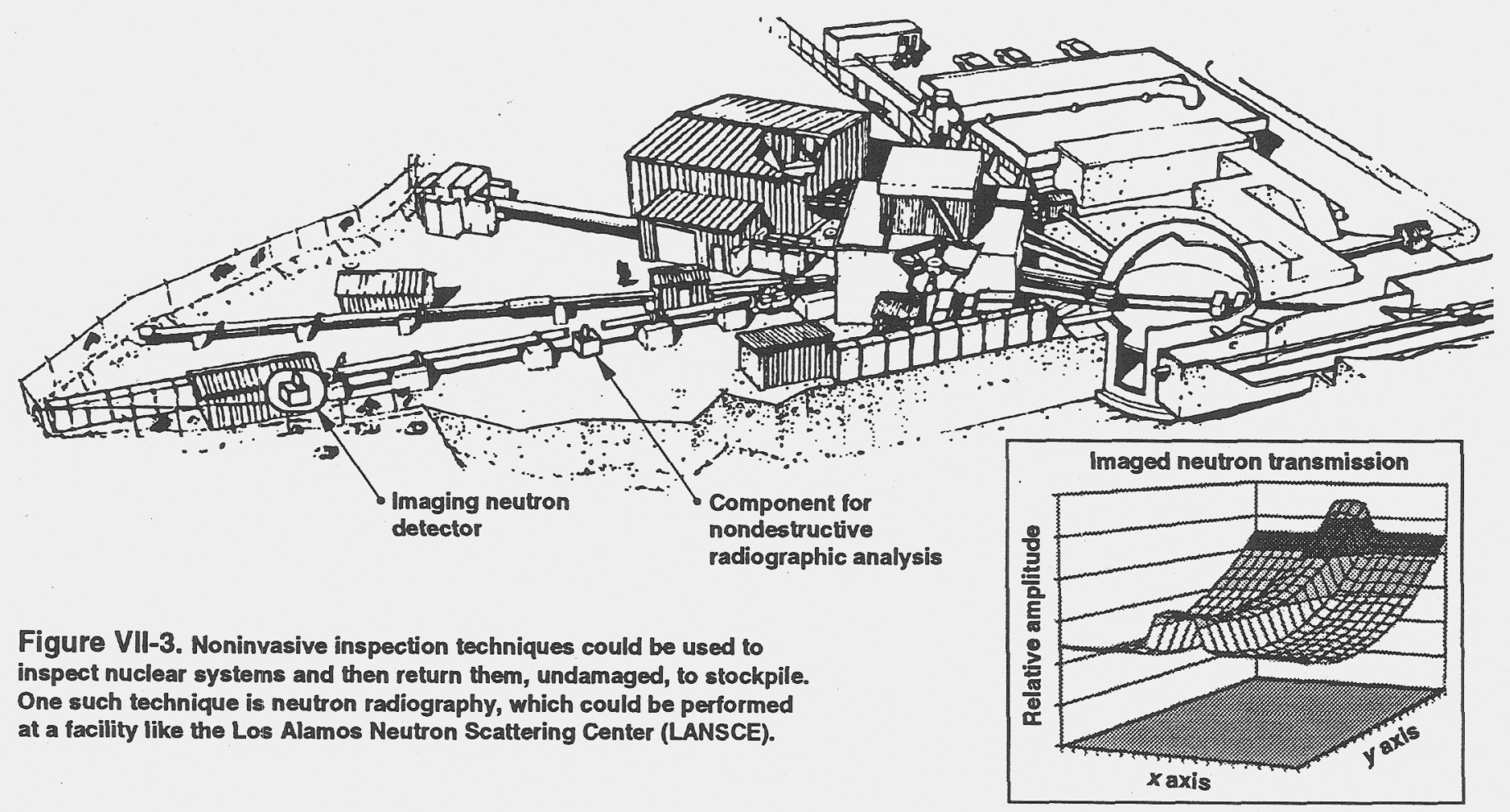


An improvement in spatial resolution is needed. High-energy neutron radiography offers new possibilities for a noninvasive, nondestructive technique to examine nuclear assemblies because high-energy neutrons:

- Readily penetrate thick layers of heavy metals.

- Can detect defects in embedded light materials (e.g., supporting plastics).

- Can detect gaps or position shifts in heavy metal components. This technique makes it possible to inspect certain nuclear systems and then return them to stockpile. The neutron radiography experiments could be performed at a facility like LANSCE (Figure VII-3). The principal issues associated with neutron radiography have to do with detector technology, and additional work is needed to increase detector sensitivity to reduce the time needed to record an image.
High-Fidelity Flight Tests

We will explore the feasibility, cost, benefits, and drawbacks of incorporating a miniature telemetry system in every weapon, either at the time of original assembly or during system modification. If this is done, the only change to the weapon that would be required for flight tests is to replace the nuclear package with mock components. The miniature telemetry system would monitor the signals to and within the warhead and provides the necessary data transmission to ground-based receivers while the weapon is in a typical delivery mode. Ground monitoring of neutron generator output and implosion initiation would be necessary to give full diagnostic data. Techniques for these measurements have been demonstrated, and further development would provide increased sensitivity and information.
Hydrodynamic Tests and Hydronuclear Experiments Changes in plutonium metallurgy, chemical transformations of high explosives, changes in the plastics and other materials surrounding the nuclear components, and other potentially deleterious changes in weapon components must be correlated with performance to assure confidence in system safety and reliability. We will perform in situ nondestructive evaluations to determine how material properties change with time. We will use periodic hydrodynamic tests and, if allowed, hydronuclear evaluations of sampled weapons, coupled with computer simulations of aged and original configurations, to correlate long-term aging effects with implosion performance of primaries. We will compare these experiments to benchmark data obtained as soon as possible, before further aging has occurred. 


\section{Computational Simulations}

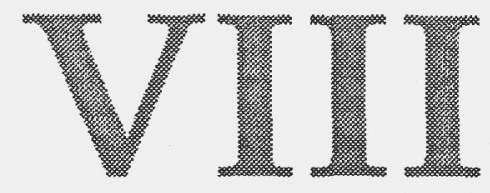

M odeling and simulation of complex systems has, historically, underwritten almost every aspect of nuclear weapons research and development.

Improved predictive models and enhanced computational capabilities are required for three reasons: to ensure confidence in the safety and performance of the existing stockpile without nuclear testing, to prepare for meeting future uncertainties, and to reduce the cost of maintaining the U.S. nuclear deterrent. These improvements are urgently needed not only to fill, as best as possible, the gap left by the nation's decision to forego nuclear testing but also to meet the requirements of numerous stockpile stewardship program elements in a timely manner. In each of the other sections of this document, the need for increased capabilities in computational simulations was noted. Here, we give specific details on the requirements for computing and simulation to meet the collective needs of the Stockpile Stewardship Program.

The following areas will require significant advances in computational simulations in order to maintain confidence in the stockpile at an affordable cost. - Nuclear components: Largescale computational simulations are used to assess the safety and performance of the nuclear stockpile. Current nuclear physics simulations are extremely sophisticated and integrate massive amounts of data with complex models of highly coupled, nonlinear physical processes at extreme pressures, temperatures, and densities. Nonetheless, even when normalized to existing experimental data and run on the most powerful computers available, they are unable to provide the predictive capability required for science-based stockpile stewardship. Dramatic advances in

\section{Computational Simulations}

Key Issues

- Because of the complexity and large number of phy sical processes involved in modeling the operation or nudear weapons: high-performance computing is essential to evaluate the safety and reliability of the stockppile.

- In the future, computational models, constrained by past nuclear tests and new nommuclear experiments, will provide the only measure of full system performance of the stodisplle.

- Advances in high-performance computing (1housamilfold increases in computer speed, memory, and storage capabilities) are needed to adlequately simulate weapons in three dimensions with models of all important physical processes.

- Improved intormation-management iools are needed to effec tively access and use the large amount of data that will be gener ated by filure computiters.

Advances in highpperformarice computing are needed to realze the fill potential or virtual girototyping and to develop appropriate predictive aging capabilities.

\section{Major Thrusts:}

- Procurre and help dive the development (in partinership wilh industry and other gov vermment ageneies) of high performance computers using parallel arehitecture to improve speed and expand nemory.

- Procuree and help dirve the commereial development of large storage systems and high-speed networks for distributed aceess? - Develop application software to exploit parallel machine archi: tecturres.

- Develop system softwane to support an integrated usert environment with improved data-comprehension and data: management tools:

- Develop improved theoretical models of important phy sical processes:

- Develop the computational tools to implement approphitate virtual prototyping capabilities, perform aging studies of materitals and components, ensure the abillty 10 remanufacture matert: als and components, and develop models for manufacturing processses. 
the accuracy, level of detail, and completeness of these simulations are needed.

- Nonnuclear components: Computational simulations are required to assess component and integrated system performance under normal operating conditions and under exposure to the wide variety of external stimuli that may be caused by credible abnormal environments. In addition, model-based design of components and their manufacturing processes will be essential for the manufacture of reliable parts in small lot sizes with first-pass success and to remanufacture parts after extended periods of production dormancy. However, to realize the potential offered by modelbased manufacturing, exacting computer-based descriptions of the multitude of complex physical and chemical systems used to manufacture materials and parts are needed.

- Stockpile surveillance: Safety and performance assessments are required for stockpile surveillance and to certify future "fixes" and retrofits; in the absence of nuclear testing, we must rely on computational simulations. We need an in-depth

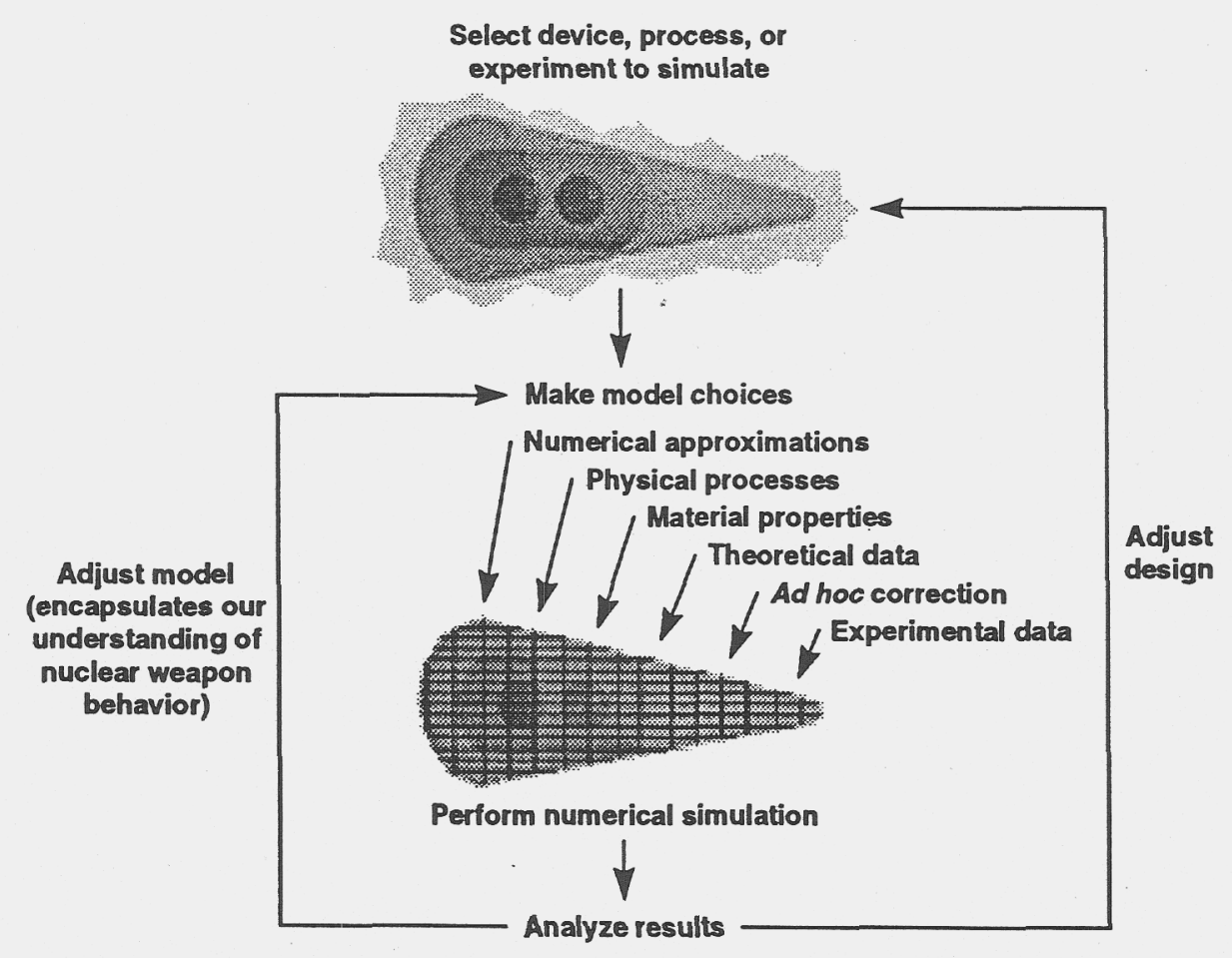

Figure VIII-1. Computational simulations using detailed numerical models are used to predict the results of physical experiments. Without nuclear tests and dedicated manufacturing facilities, evaluation of weapon safety, performance, and manufacturability must rely much more heavily on computational modeling and simulation, which must be founded on a thorough scientific understanding of the systems. understanding of the age-related changes that occur in weapon materials and the effect of those changes on material properties and, in turn, on weapon safety and performance. With this knowledge, we can construct the computational capability that will allow us to understand why components fail after periods of time in the stockpile, to predict impending failures, to redesign longer-lifetime replacement components or systems, and to guide the development of integrated "smart" sensors for stockpile surveillance.

Computer simulations are sometimes referred to as "computer experiments" because, like physical experiments, their results cannot be determined beforehand. Figure VIII-1 illustrates how simulations using a detailed numerical model of the weapon or weapon system are used to predict the results of physical experiments. The steps are the same when simulating component aging, materials compatibility, or manufacturing processes. The model includes: - A geometric description of the system.

- The system's material and physical properties.

- A set of physics processes, equations to describe them, and physical property databases.

- A set of computational algorithms.

By running a series of simulations, a designer can quickly investigate which features of a design are likely to be most sensitive to change. When a computer program is available to run such a problem, this process 
can be completed in much less time than it takes to conduct a physical experiment; this process also permits the designer to examine the effects of physics features or model assumptions that would be difficult or impossible to probe directly in a physical experiment. In fact, many of the fundamental material properties used in these models, such as material opacities or equations of state, are themselves calculated from physics equations.

An extremely close partnership exists between computer simulations and nuclear and nonnuclear experimentation. Over the years, better simulations have placed greater demands on physical experiments to measure additional physical properties and to improve the accuracy of existing measurements. In turn, better experimental data have constrained computational models, highlighting their limitations and leading to improved numerical simulations.

In the future, weapon scientists will use advanced computational tools to design and analyze new classes of nonnuclear experiments to probe relevant weapons physics issues.

Carrying out these analyses will be critical to the development and maintenance of the designer's basic skills and scientific understanding. By applying these skills to the analysis of past weapons data, preserved in a computer-based archive, future designers will be able to develop the ability to make informed judgments about weapons safety, reliability, and performance.
With the dramatic reductions planned for the weapons production complex, computational methods and virtual prototyping will assist the redesign and evaluation of weapon components for stockpile systems. These simulations eventually will provide an integrated endto-end modeling of all aspects of component design, manufacture, development testing, and system integration.

Techniques for predicting the effects of aging on materials and material interfaces in weapon components must also be developed so we can predict materials degradation and prevent adverse effects on system reliability. Multi-level modeling schemes will be required to couple microscopic and macroscopic models of materials response.

In order to computationally simulate all relevant nuclear weapon physics, analyze nonnuclear experiments, adopt modelbased design for the product realization process, and assess materials aging effects, we will need dramatically enhanced computational resources, in particular, three- to four-fold increases in computing speed and memory.

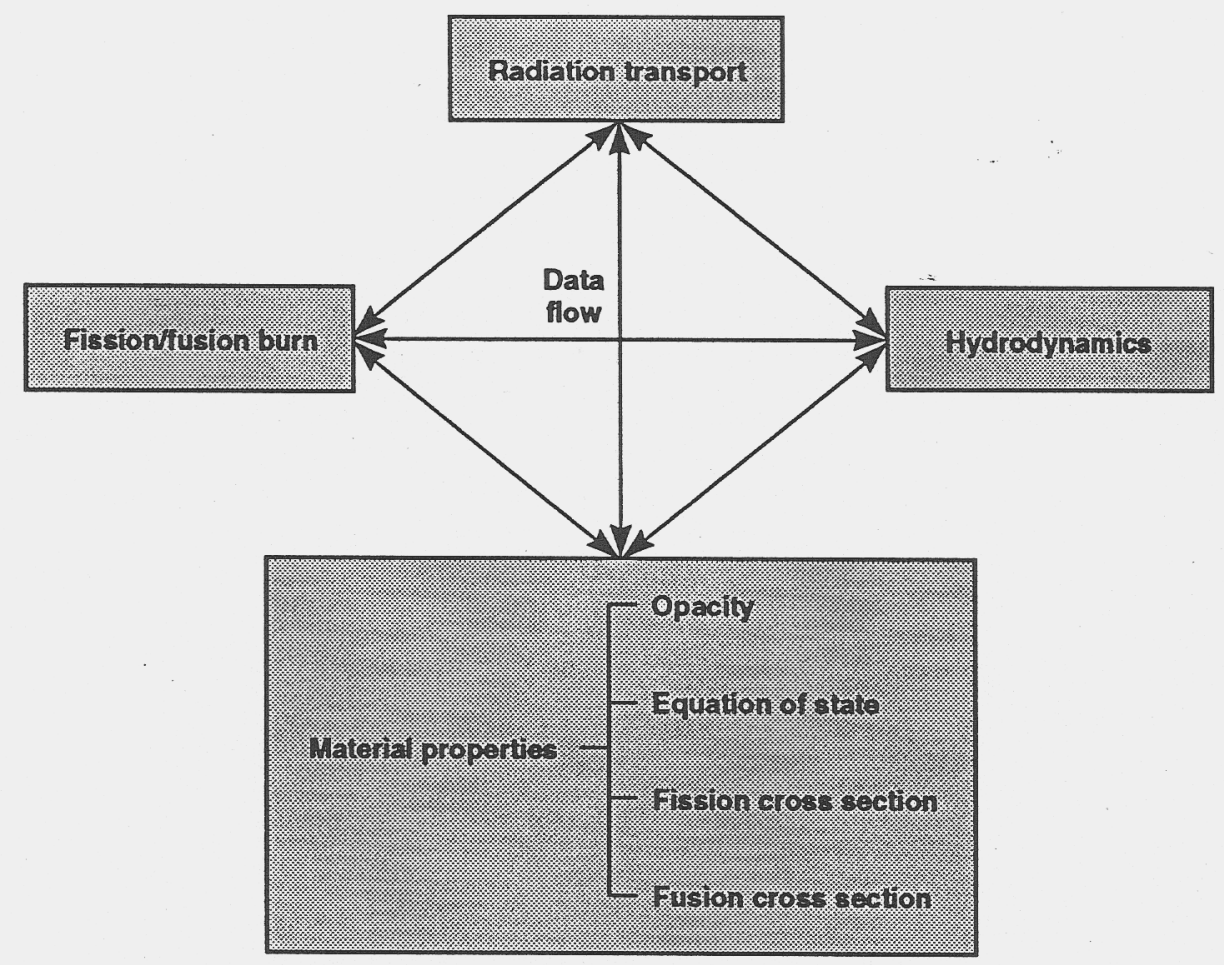

Figure VIII-2. Our weapon simulation codes are among the most complex ever developed, incorporating hundreds of physical processes in millions of lines of instructions, and require the most powerful computers in the world. Even so, they are still models, with limited ranges of validity and inadequate in known (and unknown) ways. Our simulations of systems safety and reliability, component remanufacture processes, and weapon material aging suffer from similar limitations. 


\section{Computational Simulations}

\section{Issues of Concern}

The weapons laboratories use the most advanced computational tools available for computer-based design and assessment of nuclear weapons and their manufacturing processes. Today's advanced weapon simulation codes are among the most complex ever developed and require the most powerful computers in the world. They incorporate hundreds of different physical processes in millions of lines of instructions (Figure VIII-2). Even so, these computational models of weapons are just that-models, with limited ranges of validity and inadequate in a number of known (and possibly some unknown) ways. In addition, many of the models are empirical (that is, based on experimental data), not based fully on first principles physics understanding. As a result, there are regimes in which current models are not accurate enough for final design definition or other decision-making.

Recent experimental data continue to demonstrate the limitations in our current computational models. In particular, although these models can predict many key aspects of weapon behavior in cases where we have extensive data, our ability to predict weapon response in new or unusual configurations needs improvement. For
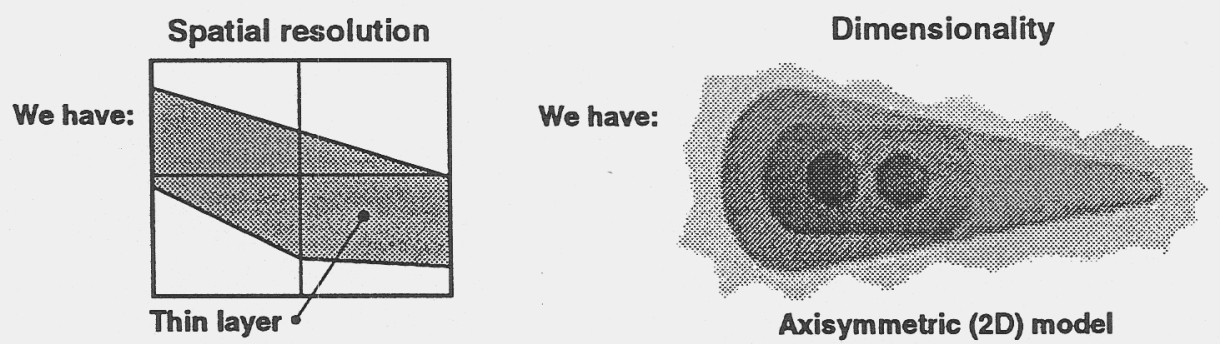

Axisymmetric (2D) model

We need:

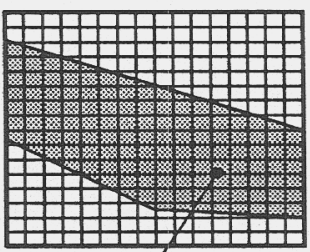

Thin layer $d$

The accuracy of almost all our calculations is limited by spatial resolution

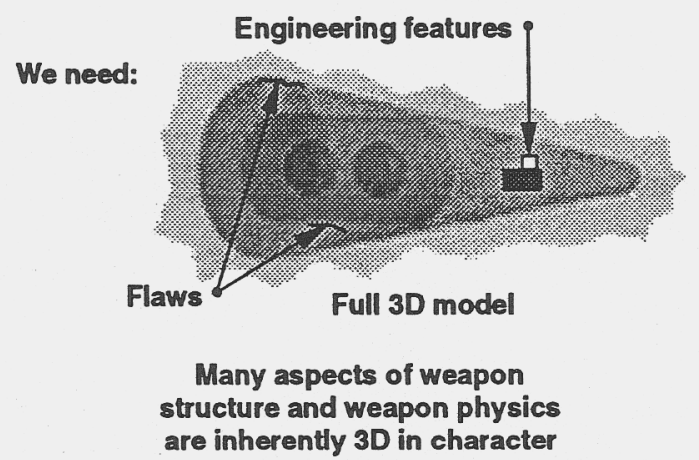

example, our best three-dimensional (3D) calculational models of nuclear safety have had to be repeatedly adjusted to agree with data from a test series conducted between 1988 and 1992. In addition, accurate 3D calculations of system response to degradations from aging or to complex accident environments, including combined thermal and mechanical insults, require significantly improved computational capabilities. The current science and technology underlying virtual prototyping is not yet mature. This simulated environment must provide the real-time response of the system and allow the entire system to be evaluated, operational planning to be done, and tradeoffs to be
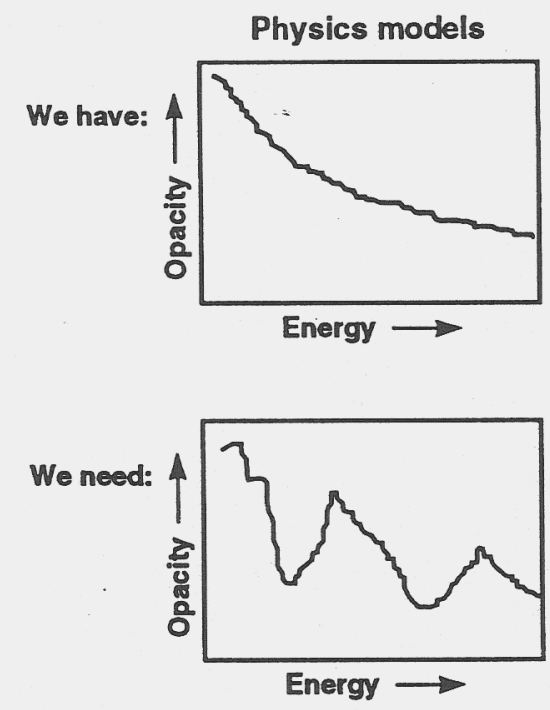

Our codes contain known approximations that obscure our understanding and limit our predictive ability

Figure VIII-3. Current computational models of weapons behavior are limited in spatial resolution, dimensionality, and physical processes. The accuracy of almost all weapon calculations is limited by spatial resolution. Many aspects of weapon structure and physics are three dimensional in character, but few of our codes can do 3D modeling. In addition, the codes contain known approximations of physics processes, which obscure our understanding and limit our predictive capability. 
analyzed. For some of the required simulations, new software will have to be developed.

Computational issues of concern are as follows:

- Without nuclear testing and with the closing of other critical testing facilities, advances in high-performance computing are essential if we are to be able to evaluate the safety and reliability of the stockpile.

- Without nuclear testing, computational models, developed from past nuclear tests and new nonnuclear experiments, will provide the only measures of full-system performance of the weapons in the stockpile.

- Advances in high-performance computing (thousandfold increases in computer speed, memory, and storage capabilities) are needed to simulate weapons and weapon systems adequately in three dimensions with models of all important physical processes and to support modelbased design and manufacturing activities.

- Improved informationmanagement tools are required to effectively access and use the large amount of data that will be generated by future computers.

\section{The Program for Addressing these Issues}

The need to markedly improve the accuracy and extent of our computer simulations drives stockpile stewardship activities related to high-performance computing.

- To run more accurate, more complete simulations, we need faster, more powerful computers, and this requires that we move to massively parallel processing (MPP).

- To exploit the potential of MPP, new codes must be written and existing codes must be reworked specifically for the massively parallel environment.

- To handle the increased quantity of data generated with the MPPs, we need faster networks and larger storage volumes. - Improved technologies for information management, data comprehension, and scientific visualization are needed for analyzing the data and providing ready access to all relevant modeling data and archives of theoretical, experimental, engineering, manufacturing, and other information.

\section{Improving Computer \\ Simulations}

Current computational models of nuclear weapons are limited in three principal ways (Figure VIII-3): spatial resolution, dimensionality, and physical processes.

- Spatial Resolution. Simulation codes divide space into a large but finite number of cells or zones. Each cell occupies a certain volume of space and has a characteristic length, which sets a lower limit on the size of the features that the code can accurately resolve. Features smaller than a cell cannot be accurately modeled spatially, while features that extend over many cells can be modeled with greater accuracy. In order to reduce the number of cells in a simulation to the point where it can be run in a reasonable amount of time (hours to days) on today's computers, designers must use cell sizes that are far too large to adequately resolve detailed phenomena and features, including:

* Material interfaces.

* Shock-wave structures.

* Instability growth.

* High-explosive detonation waves.

* Assembly gaps.

* Manufacturing variations.

* Neutron and radiation transport. opacity.

* Time- and space-dependent

* Time- and space-dependent fission and fusion processes.

This lack of resolution is a major limitation in several important simulations. For example, for the nuclear components, three-dimensional calculations of safety, the effects of aging, and the response of full weapon systems to complex environments require a characteristic cell size roughly ten times smaller than is currently used to reach the resolution needed for accurate calculations. In three dimensions, a factor-of-ten increase in resolution requires approximately a thousandfold increase in the number of zones, with at least a proportional increase in the computer time required to run a problem.

- Dimensionality. To accommodate current computational capabilities, complex 3D objects are frequently approximated by simpler ones with cylindrical or spherical symmetry, limiting the accuracy of the computer models. This is analogous to approximating an orange, with its lumpy shape, surface texture, 


\section{Computational Simulations}

and stem, as a perfect sphere. These approximations significantly reduce the number of zones used in the simulation for a given level of spatial resolution; however, they neglect any initial $3 D$ structure in a weapon as well as the 3D features that can arise during its evolution (e.g., spall or hydrodynamic instabilities) and can have major effects on weapon behavior. To improve the predictive power of our computer simulations, it is important to model both types of 3D structures.

Furthermore, virtually all credible accident scenarios (e.g., fire, crash, blast) of importance for nuclear weapons involve nonuniform thermal and mechanical loads on a system that is itself a complex 3D assembly of electrical and mechanical components. Clearly then, 3D codes, with the ability to treat coupled thermal and mechanical processes, will be required to model the various component responses and interactions that can occur and to provide confidence in the safety of the overall system under all credible abnormal conditions.

In addition, many of the problems expected to occur in the enduring stockpile will be inherently 3D in character (as weapon safety calculations are today). Evaluating the implications of these aging-related features will require a full 3D modeling capability for all phases of the performance of a nuclear weapon.

- Physical Processes. The omission or oversimplification of physical processes is the third limitation in our current codes. By making these physics approximations, the time required to run a simulation can be significantly reduced. However, to improve our predictive capabilities, some (a) Computing performance

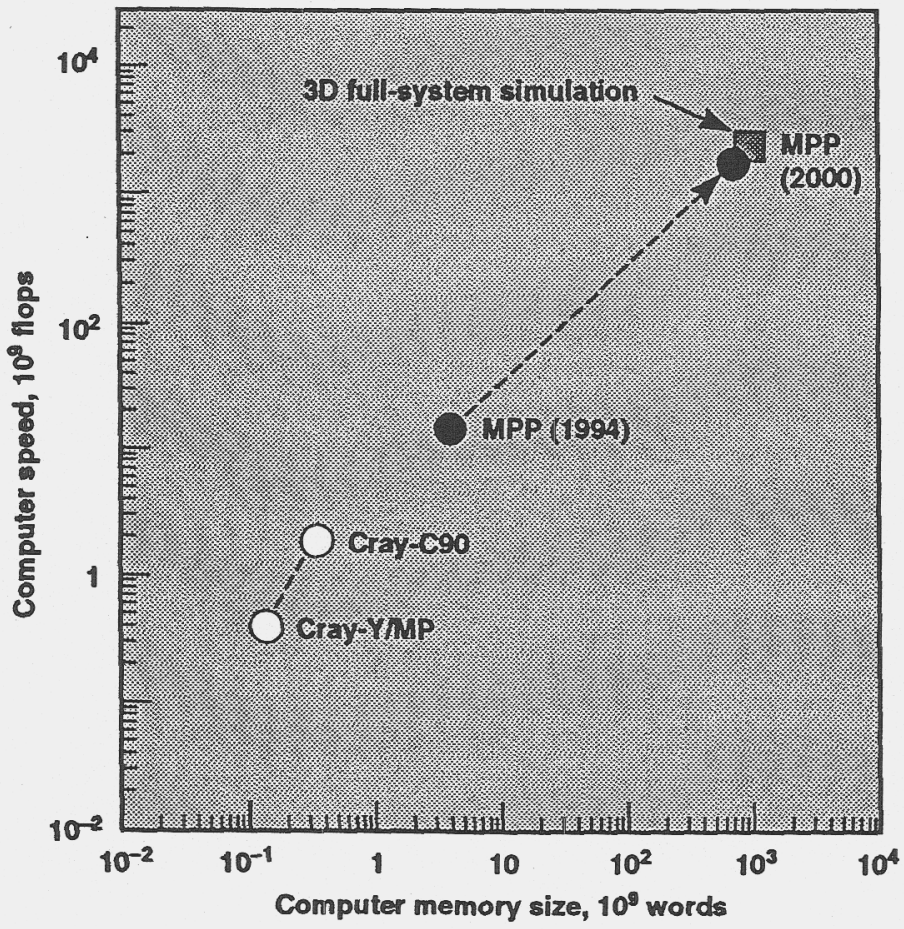

(b) Archival storage

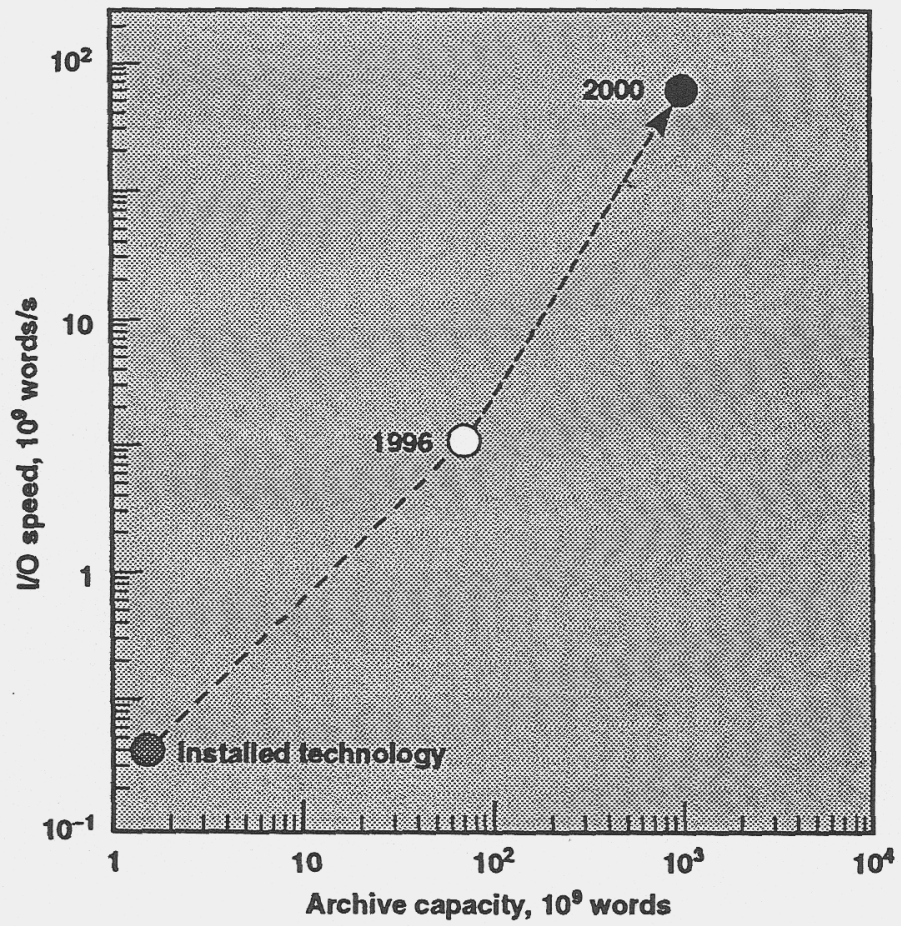

Figure VIII-4. Required advances in computing resources. (a) Projected performance curve for massively parallel processors, based on conservative estimates of expected improvements in the basic technology of these machines. The computing capabilities required to significantly improve our predictive capability should be technologically feasible by about the year 2000. (b) Network and archival storage systems must also be expanded. Gigabyte-per-second network bandwidths and VO speeds are required, and storage densities of tens of petabytes (1015 bytes) are needed, increases of several orders of magnitude. 
new physical processes must be included and others will have to be treated more accurately. Processes that will need to be considered include high-explosive deflagration-to-detonation transition, material fracture processes, shock-driven ejecta, and radiative opacities. It is difficult to estimate the additional amount of computer capability that will be needed to satisfy these requirements because, in many cases, new theoretical models will have to be developed and validated by experiments before they are inserted into the codes. We estimate that including just the most important physical processes to improve our simulations will require at least an additional factor of ten in computational capability.

Effective science-based stockpile stewardship requires the development of vastly expanded computational tools. In particular, advances in computer hardware (faster machines with more memory), coupled with improved computational techniques (for example, applications of arbitrary Lagrangian-Eulerian hydrodynamics or automatic mesh refinement) and refined physics models, offer promise for overcoming these three limitations.

\section{Hardware, System Software, and Application Software}

From the preceding discussion, we can estimate the computational capability that will be required in the future, both in terms of computational speed and in terms of the amount of memory. - Increasing Computational Speed. Computational speed must be sufficient to run a problem in hours to days, since longer times do not permit enough calculational variations to be useful in research, analysis, and design. To provide the improvements described in the previous section, we will need computers capable of providing:

* Calculational speeds a few thousand to a few tens of thousands times faster than the current best processor.

* A comparable improvement in memory relative to the current best storage device.

- Massively Parallel Processing. To obtain the required improvements in computational capability at affordable cost, we will take advantage of and help drive a revolutionary advance in computing technology, focusing the power of hundreds to thousands of microprocessors on a single problem. The projected performance curve for massively parallel processors (MPPs) shown in Figure VIII- 4 is based on conservative estimates of expected improvements in the basic technology of these machines. The computing capabilities required to significantly improve our predictive capability should be technologically feasible by about the year 2000.

- Moving Simulation Codes to MPP. We must meet a number of hardware, system software, and application software challenges to use this new generation of computational hardware. Many of our existing simulation codes (totaling upwards of 10 million lines) must be extensively revised or rewritten to take advantage of the new, massively parallel architectures. No software is capable of automatically converting existing codes to make effective use of these architectures. Indeed, the use of MPPs for many classes of problems is an evolving art. Moving our simulation codes to the massively parallel environment and adding additional physics packages will be a major undertaking and will require years to accomplish. Once converted, the new codes will have to be carefully validated against established computational and experimental benchmarks.

\section{- Expanding Computer}

Networks and Storage Systems. Computer networks and archival storage systems must be expanded to handle the increased volumes of data that will be generated by the new, more powerful computers (see Figure VIII-4). For example, network bandwidths on the order of gigabytes ( $10^{9}$ or billions of bytes) per second will be required. The $I / O$ (input/output) speeds and storage densities of our archival storage facilities must increase by two to three orders of magnitude.

The networking industry is actively developing the required capabilities, presuming that encryption at these bandwidths is unnecessary. Also being studied is the feasibility of exploiting these network capabilities to allow sharing of the computational capabilities of different sites.

However, the archival storage industry is not preparing for the needs of these new computational platforms. To address this problem, the weapons laboratories have begun a collaboration with industry and academiathe National Storage 
Laboratory - to develop and deploy the needed high-performance storage technology.

In addition, the laboratories will have to integrate other hardware and software components to provide the infrastructure for a high-performance computational capability using the new computers.

- Improving Computational Tools. Enhanced computational tools will provide a tremendous increase in the data available to scientists and engineers. Modern information-management technology, implemented in an integrated user environment, will be needed to analyze these data and provide effective access to all relevant modeling data and archives of theoretical, experimental, engineering, manufacturing, and other documentary information. Advanced data-comprehension and scientific-visualization tools will be required to evaluate this wealth of information. This integrated data-management and analysis environment will maximize the use of advanced computational tools developed to improve our predictive capability and is an essential component of virtual manufacturing (see Section VI).

\section{The Computational Environment} of the Future

The net effect of these hardware and software advances will be the development of an unprecedented but necessary capability for computationally predicting the behavior of complex systems. This computational environment of the future will provide our only means of maintaining confidence in the stockpile without nuclear testing. It will also allow the fundamental change that is needed by the weapons program for product realization at affordable cost.

The weapons scientist of the future will have the ability to

MPP-based, high-performance computing resources at locations across the country

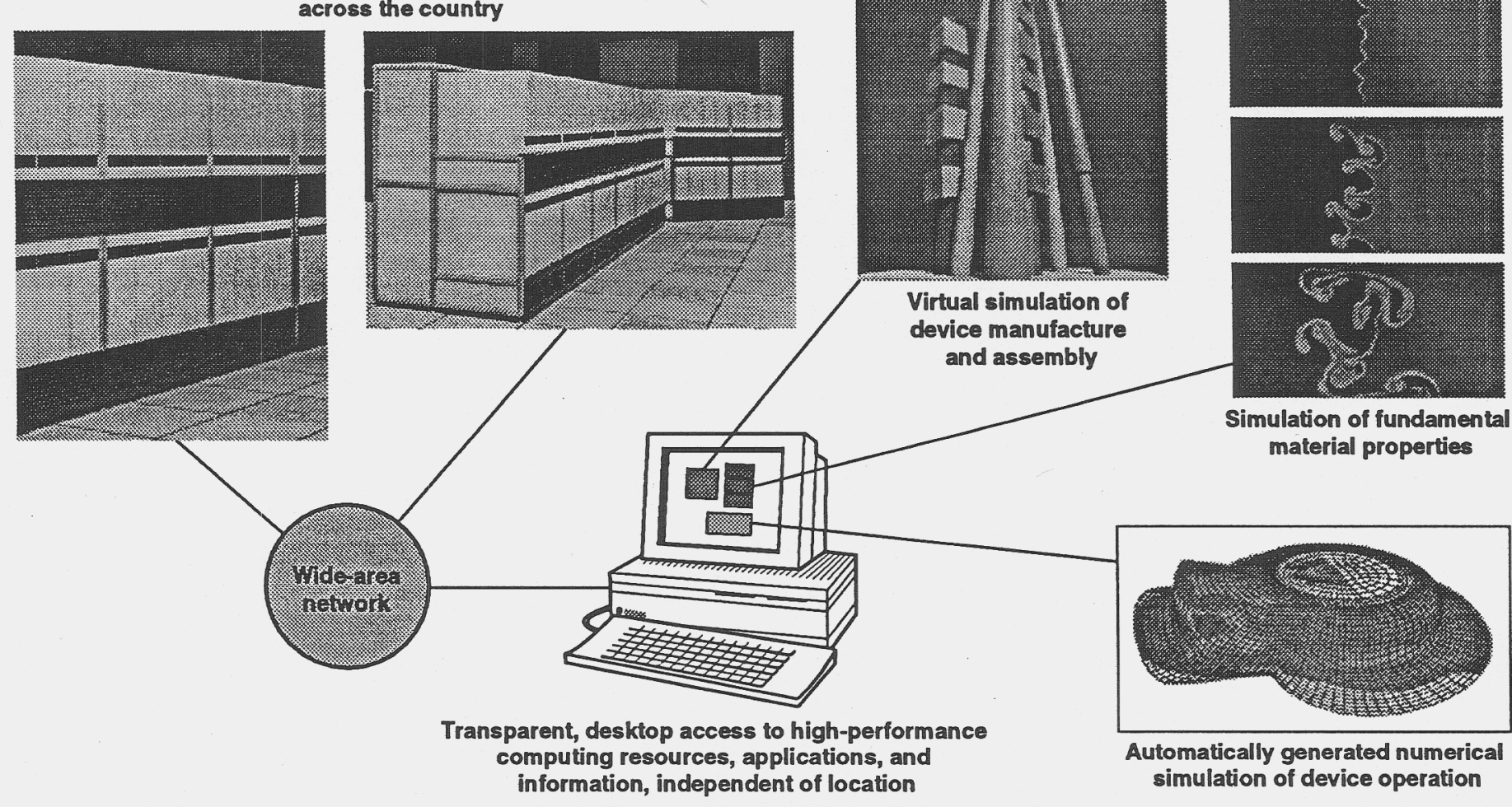

Figure VIII-5. The computational environment of the future will allow weapons scientists to draw on high-performance computing resources at a number of sites across the nation-MPPs, databases of archived test results, material properties, physics models, datamanipulation and visualization tools, etc. - to evaluate design variables and accurately predict weapon performance. The final computational model can then be fed directly to a virtual manufacturing system to finalize a part or component design for fabrication. 
rapidly construct sophisticated 3D geometric models of an experiment, component, or subsystem using the latest tools for computer-aided design and manufacturing (CAD/CAM). Automatic mesh and problemgeneration tools running on an MPP will allow the scientist to turn the geometric model into a fully specified computational model in minutes to hours, rather than the weeks to months currently required. During this process, the scientist will have online access to all relevant background data, including analyses of appropriate material models, the experiences of other scientists working on similar experiments or devices, and empirical and computational results from previous experiments (Figure VIII-5).

As a goal, the computational model itself will include all key physical processes at a level of numerical and spatial accuracy sufficient to attain the required predictive capability and will be run at teraflop rates $\left(10^{12}\right.$ or a trillion floating-point operations per second), delivering an answer in tens of hours or less. The results of the computational experiment, comprising hundreds to thousands of gigabytes of data, will be analyzed with the aid of sophisticated 3D visualization tools capable of interactively processing and manipulating the data both spatially and temporally and comparing the new data with a wide variety of experimental and calculated data. If required, the final computa-tional model will be fed directly into a virtual manufacturing system to make design/producibility tradeoffs. Major elements of the integrated computational capability will couple teraflop-class MPPs and special-purpose graphics processors, connected by a gigabyte-per-second optical network to each other, to the users, and to an archival storage facility capable of storing tens of petabytes of data at $I / O$ rates of tens of gigabytes per second or better. A sophisticated distributed computing environment will render the complexity of this resource transparent to the user, making it as easy to use as a typical workstation.

The existence of such a computational modeling capability will not only play a key role in science-based stockpile stewardship, but it will also transform the way that science and engineering are practiced in the future. Progress in virtually every branch of science and engineering will be accelerated by the availability of such an integrated set of capabilities. One must remember, however, that no amount of sophisticated computing can fully replace actual experimental data.

The development of greatly improved computational modeling and information management capabilities is essential for the science-based Stockpile Stewardship Program. Providing these capabilities will place severe demands on our computational resources, in terms of both hardware and software development. The current revolution in computing and its related technologies will allow us to meet these demands. However, no single organization has the ability or resources to address the complete set of tasks required to exploit the tremendous potential of this revolution. The weapons laboratories need to continue forging â set of collaborative relationships among themselves, other government laboratories, industry, and academia to harness this capability. In addition to directly supporting the needs of the Stockpile Stewardship Program, this effort will result in the creation of a powerful set of tools that will greatly benefit the broader scientific and technical communities. 


\section{The Nevada Test Site and Test Readiness

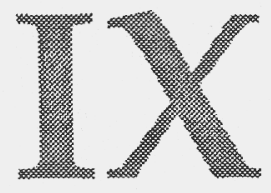

$\mathbf{I}_{\mathrm{s}}$ n July 1993, President Clinton, as part of his policy to seek a comprehensive test ban (CTB), extended the nuclear testing moratorium initiated in October 1992 while charging the DOE to maintain the capability to resume nuclear testing. In the fall of 1993, the President further defined this task to:

- Maintain the capability to conduct a nuclear test within six months up to FY1996.

- Maintain the capability to conduct a nuclear test within two to three years after that time.

President Clinton also stated that:

"Under a CTB, keeping a viable infrastructure and staff at the Nevada Test Site, the Department of Energy's nuclear weapons laboratories, and the Defense Nuclear Agency will be a fundamental requirement to maintain the capability to resume nuclear test activities. Nevada Test Site resources should, therefore, include (1) those necessary to conduct the appropriate experimental activities, (2) infrastructure that will allow for future return to underground nuclear testing, (3) those which will permit other program efforts, (4) the continuation of environmental and health-related functions, and (5) activities to assure public safety and physical protection."

A nuclear test is a complex operation that requires the coordination of many organizations, including federal agencies, private contractor organizations, and the nuclear weapons laboratories. To field a nuclear test, these organizations must work closely together to meet demanding safety, technical, regulatory, and legal requirements.

\section{Issues of Concern}

The issues of concern to test readiness involve the need to maintain the critical skilled staff, technologies, and infrastructure required to safely conduct nuclear tests in an era of uncertainty as to whether we will ever conduct another nuclear test and in which fiscal
pressures require the most effec- tive and efficient use of limited national security resources. In particular:
- Without an meaningful and challenging experimental pro- gram that uses the unique skills and infrastructure required for nuclear testing, the personal and skills required for nuclear test- ing will rapidly be lost. This concern was noted by President Kennedy during the 1958-1961 test moratorium: "...we cannot keep top-flight scientists concen- trating on the preparation on an experiment which may or may not take place on an uncertain

\section{The Nevada Test Site and Test Readiness}

Key Issues:

- The skilled staff critical technologies, and necessary inirastructure will not be retained if we do not condiuct a meaningfol and challenging experimental program at the laboratories and the Nevadia Test Site

- Support tor the infrastructure and the experimental program will be difficult to ob tain so long as their purpose is perceived to be solely one of maintaining readiness for a program that may never be conducted.

Major Thrusts

- Perform aboveground experiments at the laboratories and at the Nevada Test Site that address the need to exercise test-relat ed skils and other elements of stockpile stewardship.

- Perform undergroumd experiments at the Nevada Test Site that addrress the need to exereise the rest-related skills and other elements of stockpile stewardship:

- Help develop new customers for large-scale test and demon stration activities at the Nevada Test Site in support of other. national missions that will help keep current the testing and experimental infrastructure.

- Identify and preserve eritical saffety - related nudelear testing skills and knowledge by interviewing test-experienced persons Who plan to leave or have left the testing organizations and by recording the information in a consistent easily retrievable form 
date in the undefined future. Nor can large technical laboratories be kept waiting for some other nation to break an agreement."

- As long as the experimental program and infrastructure support are viewed as existing solely for the purpose of maintaining a readiness program that may never be conducted, the support and funding for the program will quickly disappear. A Presidential mandate to retain readiness will be no more effective than it was for readiness to resume atmospheric testing. This problem has already manifested itself in last year's congressional actions on test readiness funding.

- An additional concern relates to maintaining the Nevada Test Site. Historically, it has been viewed-incorrectly-as only a nuclear testing site. As a result, it has become a symbol of nuclear testing, and its closure is viewed by some as an essential element of a comprehensive test ban.

\section{The Program for Addressing these Issues}

To maintain the necessary skills and infrastructure for nuclear testing, the DOE will perform other types of experiments and other stockpile stewardship activities that utilize critical nuclear test skills and facilities while addressing other problems of national importance. The goal is to maintain readiness as a byproduct of these other programs while minimizing the direct readiness costs. In particular, the test readiness efforts will entail:
- Aboveground experiments at the laboratories and the Nevada Test Site.

- Underground experiments at the Nevada Test Site.

- Dual-use projects at the

Nevada Test Site that support other customers.

- Archiving of the critical nuclear testing skills, experience, and knowledge, particularly as related to the safe conduct of nuclear tests.

\section{Aboveground Experiments Many testing skills can be applied in the aboveground experimental programs at the weapons laboratories and the Nevada Test Site. Science-based stockpile stewardship will pro- vide many opportunities, espe- cially at the laboratories, to exer- cise test-related skills. Some experiments envisioned in the Stockpile Stewardship Program will require, for safety or envi- ronmental reasons, the attributes of a remote test site. In those cases, the Nevada Test Site will be used; for example, hydrody- namic experiments that use large amounts of high explosives to be conducted at there.}

\section{Underground Experiments As part of the Stockpile}

Stewardship Program, we are planning hydrodynamic experiments to evaluate and assess the characteristics of primary-stage implosion using plutonium. These experiments, which will be conducted underground at the Nevada Test Site, will provide valuable information about the safely and reliability of the stockpile.

Additionally, hydronuclear experiments, if permitted within the scope of a test ban or moratorium, would be conducted underground at the Nevada Test Site as an important element of the Stockpile Stewardship Program.

Nuclear treaty verification experiments have been and will continue to use the Nevada Test Site to develop and test sensors and techniques for verifying treaty compliance. These efforts will contribute to both verification technology and transparency. Because underground experiments present some of the same observables as a nuclear test, transparency measures will be taken to address treaty compliance concerns.

\section{Large-Scale Tests and Demonstrations}

The Nevada Test Site has historically provided the nation with a test bed for large-scale hazardous experiments and demonstration projects, especially projects involving nuclear materials, large amounts of explosives, and large chemical releases. Its remote location and test- and safety-related infrastructure make it an ideal location for many of these experiments. Additionally, the adjacent military facilities and extensive buffer zone make it unique in its ability to support national security projects.

To preserve this unique infrastructure for both readiness 
and as a unique national test range, the DOE will work with other agencies to expand workfor-others programs to more fully and effectively utilize the capabilities of the Nevada Test Site. Currently, other agencies are using the Nevada Test Site for experiments related to nonproliferation, counterproliferation, treaty verification, and chemical safety. The DOE will build on these efforts to demonstrate the unique capabilities of the Nevada Test Site and to expand the test site's customer base, which, in turn, will help support skill utilization and infrastructure costs.

\section{Archiving}

We have already begun to identify skills and knowledge critical to the safe conduct of underground nuclear testing and plan to identify individuals (those presently employed as well as those who have left the laboratories) who possess critical nuclear testing skills and knowledge. We plan to use interviews and other methods to elicit and record the experiences and knowledge of these people, particularly as related to nuclear testing safety, in a consistent and easily retrievable format. As new personnel become qualified in critical functions and key positions, they will be added to this review process. In addition, we plan to identify those persons possessing critical nuclear testing skills and knowledge and record that information before they retire or leave the test organizations. Such an archive of nuclear testing experience will be vital for training new staff so we can resume testing if necessary without "reinventing the wheel." 


\section{Technology Partnerships with U.S. Industry

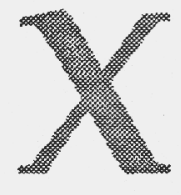

W ith the end of the Cold War, the affordability of nuclear weaponry has become a much more important consideration. To reduce the cost of designing, engineering, and producing replacement and upgraded components for current stockpile weapons as well as for potential replacement weapons, the nuclear defense and privatesector technology and supply bases must become more highly integrated with each other. To this end, the nuclear weapons complex of laboratories, test sites, and plants must eliminate historical barriers and implement new business practices. The Defense Programs Technology Partnership Program, an element of the Stockpile Stewardship Program, is an important mechanism for effecting this transition. This program supports and coordinates the development of dual-benefit technologies through cost-sharing agreements, thereby facilitating the integration of the technology and manufacturing bases of the nuclear weapons complex with those of the private sector. The Technology Partnership Program is also designed to help the weapons laboratories, test sites, and plants maintain the core nuclear weapons competencies that form the foundation for the Stockpile Stewardship Program.

The historical approach to product realization (from design, through manufacturing and assembly) taken by the nuclear weapons complex was developed for much larger production volumes than those needed in the future and without due regard to the use of commercial parts and practices. Clearly, there will continue to be many areas in which the nuclear weapons laboratories and plants must maintain the capabilities for technologies and products that are largely uncoupled from the civilian economy. However, the nuclear weapons technology and supply bases must be integrated, to the maximum extent possible and practical, with those for civilian, commercial, and other military applications. Some of the steps required to effect this transition are discussed in other sections of this report, most notably those covering the design (Section IV) and manufacturing (Section VI) of nonnuclear components. Here we briefly cover the areas of focus and the management philosophy of the DOE's Defense Programs cost-shared partnerships with U.S. industry.

The major thrusts of the Technology Partnership Program apply broadly to many industrial sectors, including:

- Advanced manufacturing, with an emphasis on agile and modelbased manufacturing.

- Rapid prototyping.

- Microelectronics and photonics.

- Advanced materials and explosives.

- Integrated microsensors and intelligent controls.

- Advanced computing and simulation of complex systems.

- Information management.

- Low-waste manufacturing.

- Waste characterization and processing.

\section{Technology Partinerships with U.S. Industry}

Key Issues

- Cost-shared research and development activities are needed to address stockpile stewardiship objectives and high-prierity industry needs:

- Cost-shared researdh and development can reduce he cost of nuclear weapons tedhnelogy componnents, and subsystems and. at the sarne time, provide an effictent nechanism of transferting valuable technologies to U. S. industry

Major Thrusts

- Develop standards tor assessing the guality or various projects: and progians and their value to the nutelear weapons program and U S indiustry

- Coordinate with other fedleral agencies the major programs

that have countemparts funded will those agenneles.

- Fully integrate the program with other stodkpile stewardistip programs. 
The activities of the Technology Partnership Program fall within four focus areas spanning the range from multilaboratory partnerships with industrial consortia to assistance to small businesses:

- Programs focused on a particular sector of industry and carried out by several laboratories and plants.

- Laboratory partnerships focused on specific core competencies of a laboratory; these partnerships may involve one or several laboratories and one or several companies.

- Small business assistance, usually performed by a single laboratory assisting a single company.

- Facilities partnerships, usually performed by a single facility (i.e., a production plant or test site) working with a single company. Further discussion of the rationale behind this Technology Partnership Program as well as discussions of the overlapping and complementary interests and capabilities of the Stockpile Stewardship Program and U.S. industry is presented in Section XI.

\section{Issues of Concern}

The principal issues for the Technology Partnership Program have to do with effectively implementing cost-shared research and development activities that address both stockpile stewardship objectives and high-priority industrial needs. Fundamentally, the objectives of this program are to reduce the cost of realizing nuclear weapons technology, components, and subsystems while simultaneously providing an efficient mechanism for technology deployment for applications other than nuclear weapons. Specific issues include:

- Ensuring a balance of benefits to the nuclear weapons programs (Stockpile Stewardship and Stockpile Management) and to national economic growth. - Effectively integrating these technology transfer programs into the nuclear weapons program and into overlapping industrycost-shared programs supported by other federal agencies.

- Reducing the time needed to initiate new cost-shared projects with the private sector. 


\section{Stockpile Stewardship and Other National Needs}

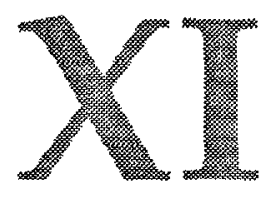

$\mathbf{T}$ he Stockpile Stewardship Program is structured to enable the industrial, scientific, and government communities to work together on mutually beneficial and cost-effective efforts to meet other national goals, especially counter- and nonproliferation and economic competitiveness. The following subsections summarize past, ongoing, and potential interactions of common interest to stockpile stewardship and to one or more other national objectives. These interactions are identified by community but may simultaneously involve more than one community. For more complete information on technical and scientific content, see the Appendix (p. 77).

\section{Industrial Partnerships}

The Stockpile Stewardship Program and U.S. industry share similar problems and complementary strengths (Figure XI-1). While the nuclear weapons program of years past demanded a basic and applied science and technology base unmatched in a single industrial laboratory, the Stockpile Stewardship Program requires an even deeper understanding of the science underlying critical nuclear weapons components and assemblies. At the same time, U.S. industry has been cutting back its investment in science and technology due to financial pressures, although long-term competitiveness clearly requires such investment. As a result, the opportunities for mutually beneficial interactions with industry have never been greater than they are now.
The motivation for industrial interactions and partnerships is discussed in Section X. Such interactions are a requirement of DOE's Defense Programs Technology Partnership Program, which is a component of the Stockpile Stewardship Program but not limited to stockpile stewardship. In fact, substantial dualbenefit interactions with U.S. industry predate the Technology Partnership Program; these interactions are increasing and involve almost every facet of stockpilestewardship-related research and development activity.

Overlapping and complementary interests and capabilities of the stewardship program and industry are discussed in other sections of this document. The areas where there is an overlap of interest include:

- Complex experimentation methods and advanced diagnostics.

- Information surety.

- Risk-assessment methodologies.

- Agile manufacturing and virtual prototyping.

- Microelectronics and photonics devices.

- Integrated microsensors.

- Noninvasive materials analysis.

- Computational physics, advanced computations, and simulation of complex systems.

\section{Counterproliferation and Nonproliferation}

To prevent the spread of nuclear weapons capabilities to other nations, we must understand their design, testing, production, deployment, and employment. The Stockpile
Stewardship Program provides a technical basis for efforts to assess, stop, or even roll back the spread of nuclear weapons and to deal with nuclear proliferation by maintaining key weapons know-how and the related scientific and engineering expertise. Specific areas in which the Stockpile Stewardship Program supports counterproliferation include:

- Understanding of weapon design and manufacture.

- Engineering and materials.

- Support for treaty negotiations.

- Proliferation-detection techniques.

- Response to nuclear emergencies.

- Technology testing and demonstration.

\section{Department of Defense}

The DOE has been a major contributor to the nonnuclear technology development of the DoD through its nuclear weapons technology base. This contribution has been manifested in a wide range of supporting technologies, fielded armaments, and devices in advanced stages of development. Even spinoffs of the technology base have resulted in symbiotic spinbacks in that challenging programs at the DOE national laboratories have enriched and maintained DOE capabilities. Specific examples of multibenefit relationships include:

- Design and integration of complex systems.

- Implosion dynamics.

- Simulation and modeling of complex systems. 


\section{Stockpile Stewardship and Other National Needs}

- Data management and communications.

- Advanced materials.

- Nondestructive testing.

- Explosives and initiation systems.

- Electromagnetics.

- Laser technology.

\section{Fundamental Science}

Science-based stockpile stewardship depends on the development of a fundamental scientific understanding and predictive capability related to nuclear weapons. To meet this challenge, we must engage in a broad range of scientific and technical activities and develop powerful, often unique experimental and computational capabilities that will allow researchers to push the frontiers of scientific research. The

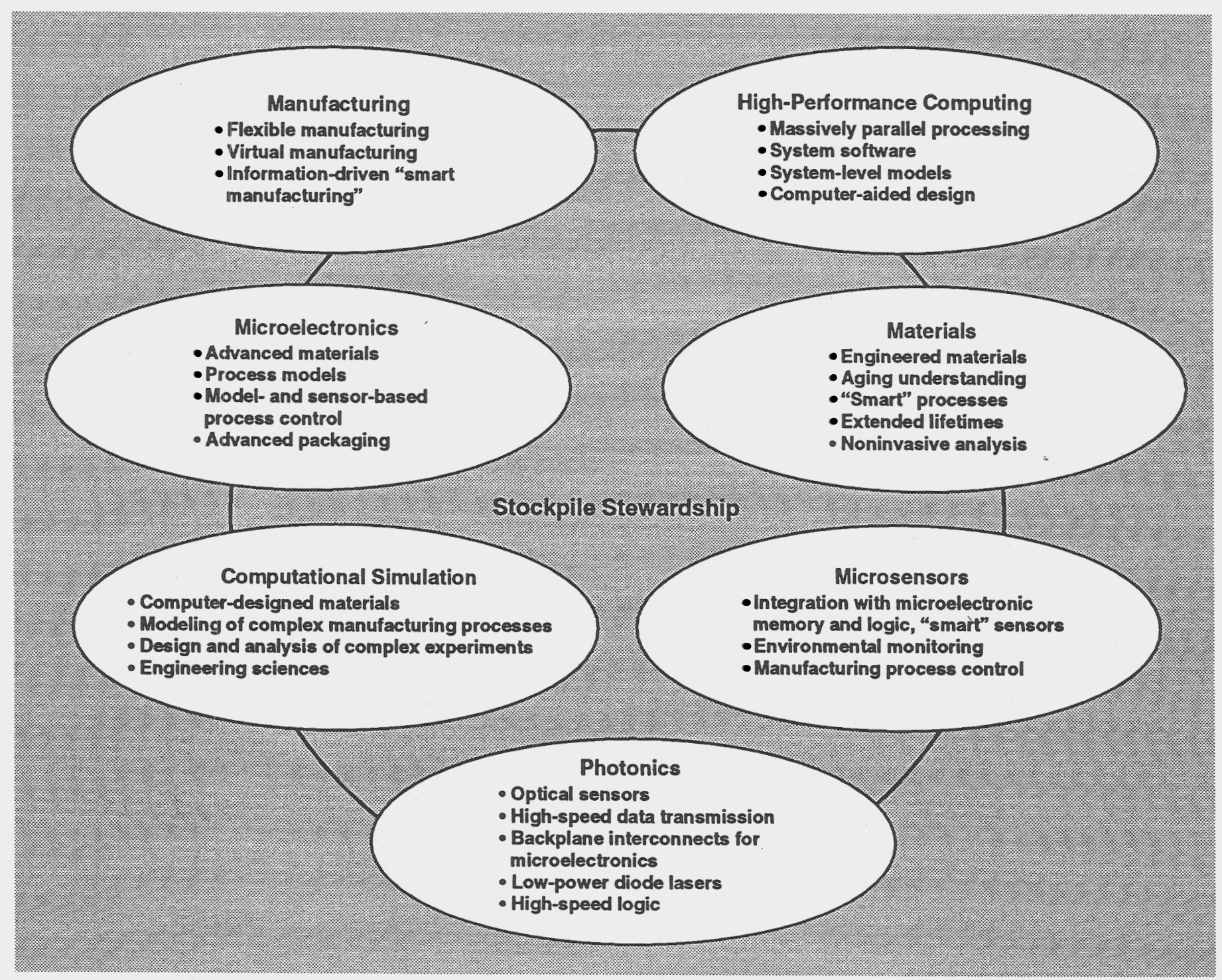

Figure XI-1. Many of the high-priority thrusts of the Stockpile Stewardship Program can directly benefit U.S. industry. By sharing expertise, facilities, and research and development costs, the nuclear weapons program can cost-effectively meet its needs and U.S. industry can develop new capabilities, processes, and products. 


\section{Stockpile Stewardship and Other National Needs}

following are a few external scientific and civilian applications where stockpile stewardship activities will likely have a significant impact:

- Astrophysics and space physics.

- Hydrodynamics.

- Condensed-matter science.

- Microelectronics and photonics science.

- Plasma and atomic physics.

- Intense pulsed x-ray sources.

- Fusion and other energy research.

- Environmental monitoring and science.

- Nuclear science.

\section{Experimental Facilities}

The DOE has been the sponsor of many "big science" experimental facilities that were available both to DOE programs and to external collaborators and partners. However, in the Cold War environment, many of the national security facilities were not generally available to outside programs, and those that were available were not widely marketed to attract other users. In the current national-security environment, the need for secrecy has been reduced and increased emphasis is being placed on cost-effective research and development and enhancing U.S. economic competitiveness. As a result, the DOE weapons laboratories can work more closely with the U.S. industrial, academic, and government communities. A number of the DOE experimental facilities and test ranges are unique in their ability to handle hazardous and energetic materials, and thus offer a unique capabilities to a new community of users.

In order to support cooperative research and development agreements, the laboratories have begun to rearrange their security areas so that new users can be brought onto the laboratory sites. These efforts will be expanded to accommodate increased collaboration in science-based stockpile stewardship activities.
The Nevada Test Site also has potential for use to address issues of national importance. It is unique in its combination of remoteness from populated areas, its experience in handling hazardous materials, its wellcharacterized environment, its proximity to the scientific and technical resources of the weapons laboratories, and its testing infrastructure. These assets, combined with the adjacent DoD capabilities, make the Nevada Test Site particularly well suited for counterproliferation and nonproliferation experiments and demonstration activities. Applications for which the Nevada Test Site will likely have a significant impact include the following:

- Weapons system tests and demonstrations.

- Test facilities for high explosives and nuclear material.

- Large-scale chemical tests.

- Conventional weapons tests and demonstrations. 


\section{Appendix}

$\mathbf{T}$ he Stockpile Stewardship Program encourages mutually beneficial interactions with U.S. industry, the civilian science community, the Department of Defense, and other government agencies. Many of the problems facing these communities are similar to those of stockpile stewardship. Integrating the scientific and technological capabilities required for stockpile stewardship with the broad science and technology bases provided by these other communities is both a national and a stewardship imperative.

Effective integration of technologies and capabilities will not occur without cormmitment. Every project in the Stockpile Stewardship Program must be examined to identify the potential for collaboration, and every teaming arrangement should strive to maximize both the benefit to stockpile stewardship and to other national goals.

The national weapons laboratories have many years of experience in developing scientific understanding and leadingedge technology and then applying them to deliver a "product." The unique capabilities of the weapons program, developed in this science-based approach to problem-solving, date from the Manhattan Project and will continue through years of sciencebased stewardship of the U.S. nuclear stockpile. The Stockpile Stewardship Program requires that the laboratories engage a broad range of scientific and technical challenges and continue to develop powerful and often unique experimental and computational capabilities; these capabilities can be brought to bear on other science and technology issues, both fundamental and applied, of importance to the nation.

Nuclear weapons development drove an extraordinary breadth of basic science and technology. The challenge of science-based stockpile stewardship will undoubtedly create new imperatives that will have farreaching effects on civilian science and technology. For example, the advances in computations (e.g., 3D modeling of complex systems) required for stewardship in the absence of nuclear testing will find many applications in areas far removed from weapons.

The weapons laboratories have a history of collaborative activities and user programs that make their expertise and facilities available to the wider scientific community. The capabilities associated with stockpile stewardship are already being applied to science and technology issues of national importance, including environmental monitoring, fusion and other energy technologies, high-speed information networks, and global climate modeling.

Stewardship itself develops and maintains expert judgment in nuclear design and technology that is essential for counterproliferation activities. The collection of computational, modeling, scientific, and testing capabilities developed for nuclear weapons has also been of advantage to the Defense Department for nonnuclear- defense-related research and development, so much so that a significant fraction of the laboratories' activities have been devoted to such tasks.

In the following sections, we identify areas of interactions with other agencies and communities that should be continued, enhanced, or initiated. For the sake of clarity, this is done serially, even though the interactions will often involve more than one of these communities.

\section{Industrial Partnerships}

The Stockpile Stewardship Program and U.S. industry share a number of common areas of interest.

- Surety. Information surety is required for coding, recoding, and accessing permissive action links (PALs), as discussed in Section IV. Information surety is also required in the new, electronically integrated nuclear weapons complex to prevent a security compromise at one site from propagating to another. Surety demands that all aspects of privacy and data integrity (including multilevel access, secure time sampling, and audit trails) be considered. These same features are important to private companies working cooperatively with other companies and for the security of electronic financial transactions, including the effect these financial transactions might have on law enforcement. The medical industry is interested in the security of medical files, and the Department of the Treasury wants assurance that transactions 
approved for large-scale use do not pose significant security risks.

- Risk Assessment. Riskassessment methodologies developed in the nuclear weapons program are applicable to the safe and secure transportation, packaging, and storage of hazardous and high-value goods. Industry is gaining interest in the use of high-security methodologies for assessing high-consequence security violations of banks and similar facilities.

- Product Realization. Flexible manufacturing will require the development of advanced manufacturing processes and technologies of mutual interest to U.S. industry. The development of smart processes (Section VI), as well as model- and sensor-based process-control activities, is an important part of such manufacturing. Plasma techniques for surface modification are representative of another class of dualuse technologies. The wide dispersion and effective use of such information will enable the development of advanced manufacturing concepts in which highly interwoven information networks produce the right product at the right time. By teaming their efforts, the Stockpile Stewardship Program and U.S. industry can share both the costs and benefits of this endeavor.

- Microelectronics. Microelectronics fabrication is a most demanding and complex manufacturing process. Tens of millions of transistors with feature sizes less than the wavelength of visible light are located on a square- centimeter wafer. Many microelectronic devices in the stockpile are highly specialized and must tolerate extremely harsh environments, which requires that they be produced by highly specialized processes. These devices are produced in low volume, and thus some industry practices are marginal for nuclear weapon production. For this reason, the nuclear weapons program has been trying to develop a deeper understanding of the fabrication processes so they can be controlled more precisely by predictive models of the process and feedback control. There are many opportunities to combine the laboratories' ability to understand and model complex systems with industry's ability to realize cost-effective equipment. Critical processes that must be better understood include plasma etching, reactiveion etching, chemical vapor deposition, and molecular-beam epitaxy. Industry is also teaming with the national laboratories to develop process models of plasma etching and chemical vapor deposition. These are indispensable processes for making highdensity microelectronic devices, but they are difficult to control to the precision required for highyield production of good devices. - Lithography. Lithography is used to define the complex patterns of resistors and interconnecting conductors. The laboratories' expertise in $x$-ray sources, high-average-power lasers, and micromachining is playing a key role in the national effort to develop x-ray lithography.
- Photonics. Photonic devices are widely used in commercial products and have numerous potential applications in nuclear weapons. Applications include high-speed telecommunications and data transmission, image processing, power transmission, optical memories, and high-speed logic. In principle, a photonic device can perform any function performed by an electronic device and may offer distinct advantages, one of which is a high tolerance to electromagnetic interference and damage. Photonics are also the bases of many smart sensors. Opportunities to partner with industry include the development of optical-based smart sensors and high-speed optical data links, made possible by the use of compact vertical-cavity arrays and integrated photodetectors and modulators for optical interconnections between high-density multichip models.

- Advanced Materials.

Advanced materials are critical to extending the life of nuclear weapons and for predicting what role material degradation plays in the functional failure of devices. We must also team with U.S. industry to ensure a reliable supply of materials and components such as specialty metals, ceramics, and electronics.

Examples of ongoing, dual-benefit projects in materials include the. Specialty Metals Processing Consortium (13 metals suppliers and equipment manufacturers working together to improve superalloy production), a cooperative research and development agreement (CRADA) with General 
Motors-AC Rochester to develop high-reliability electronics for severe environments, and a CRADA with nine companies in the Association of American Ceramic Component Manufacturers to improve the reproducibility of ceramic components. - Systems Design. Model-based systems-level designs developed in the Stockpile Stewardship Program to help system engineers make design choices and to supplement developmental testing to reduce costs will have general applications to commercial systems. These models will help streamline the design to production cycle and serve as a means of archiving design practices and manufacturing processes. The nuclear weapons program must invest in this area because of the lack of active development programs and the potential for permanent loss of a unique knowledge base. Advances in systems-level modeling capabilities will be of great interest to industry because they can reduce the time and cost of producing products.

- Virtual Prototyping. Virtual prototyping involves the complete simulation of product design, performance, and manufacturing processes before any hardware is produced. It requires concurrent engineering and productionanalysis tools that can simultaneously design a product and the processes needed to build it. An integral part of virtual manufacturing is distributed computing for modeling and simulation to address all phases of design and manufacturing and to provide direct links to sensor-based process control of the most demanding processes. This new collaborative engineering and manufacturing environment will involve a broad spectrum of industrial partners in the manufacturing of nuclear weapons and will be made available to U.S. industry.

- Surveillance Technologies. Microsensors made by combining semiconductor microsensors and integrated circuits on the same chip to produce smart sensors that have decision-making, dataprocessing, conflict-resolution, and communications capabilities are a rapidly growing segment of the sensor market. These microsensors will be developed in the Stockpile Stewardship Program for stockpile surveillance applications to permit real-time monitoring of age-related signatures in weapons and for sensorcontrolled, model-based adaptive process control in manufacturing. Commercial interest in these microsensors is great because they are less expensive, more compact, and more reliable than the devices they replace. Potential commercial applications include monitoring pressure, acceleration, temperature, oil quality, and exhaust products in automobiles and sensing hazardous chemicals in the workplace, as well as monitors for medical applications and manufacturing. - Materials Aging. Understanding materials aging, including the problems caused by dissimilar materials in contact with each other, is an important element of the Advanced Surveillance initiative and of great interest to industry. The Stockpile Stewardship
Program will focus on materials used in nuclear weapons or design choices for nuclear weapon components. Some of these materials are used in commercial products and are of direct interest to industry. More important, the understanding developed in these aging studies is transferable in some degree to other materials and materials systems.

- Noninvasive Materials Analysis. Noninvasive materials analysis with facilities like Los Alamos Neutron Scattering Center (LANSCE) and highenergy $x$-ray diagnostics with improved detectors offers new possibilities for detecting flaws and residual strain in manufactured materials without destructive disassembly. LANSCE's capabilities are unmatched in the U.S., and facility improvements needed for stewardship will also greatly increase its attraction to industry.

\section{Counterproliferation and Nonproliferation}

The Stockpile Stewardship Program supports counterproliferation and nonproliferation in several areas.

- Physics Design Knowledge. The Stockpile Stewardship Program maintains the base of physics design knowledge that is needed to understand the possible routes to weapons and the capabilities of foreign nuclear weapon programs. When models of possible proliferant nucleardevice designs are combined with available information, they 
can provide insights into the performance, weaponization, and surety status of these weapons. - Engineering and Materials. Expertise in engineering and materials is needed to analyze the signatures of active foreign nuclear weapons programs, two visible signs of which are nuclear and hydrodynamic testing. Although the Stockpile Stewardship Program does not emphasize the nuclear fuel cycle, a knowledge of the properties of weapons-usable nuclear materials is the key to identifying diversion possibilities and techniques for necessary safeguards.

- Support for Treaty Negotiations. The weapons program has provided support for many treaty negotiations. Weapons scientists have supported the Nuclear NonProliferation Treaty, most recently through strong involvement in the Iraq inspections and by providing training and equipment to International Atomic Energy Agency (IAEA) inspectors. They provide technical expertise for nuclear test ban talks, including the current comprehensive test ban talks, and the knowledge crucial to the verification of these treaties. They also provide weapons expertise to establish nuclear export control lists.

- Proliferation Detection Techniques. Many proliferationdetection technologies (the earliest of which are nuclear test detonation and debris analysis) originated from and are supported by elements of the Stockpile Stewardship Program. Many nuclear-detection capabilities were first developed for the weapons program. Today, the IAEA and the Chemical Warfare Convention are using nuclear and chemical forensic techniques for weapons. Remote sensing techniques using lidar (a laserranging device) capitalize on the laser programs, including the inertial confinement fusion (ICF) program, supported by the nuclear weapons program. - Response to Nuclear Emergencies. The Stockpile Stewardship Program provides the capabilities needed to respond to nuclear emergencies and to deter and defeat some proliferation threats. Weapon design expertise is also needed to assess the threat from terrorist weapons and to develop disablement techniques. Nuclear test expertise helps with weapons searches and in determining the origin of threat devices. The Stockpile Stewardship Program also provides some weapon-accident capability to the nuclear inheritor states of the former Soviet Union to assure that their weapons and materials are adequately protected from diversion or theft in the event of a nuclear emergency.

\section{Department of Defense}

The technology transfer programs of the weapons laboratories have evolved to dualand multiple-benefit programs that apply the core competencies of nuclear weapons stewardship to other national security needs, including the enhancement of nonnuclear defense capabilities. - Design and Integration. The design of both nuclear and conventional weapons is characterized by an iterative process of design concept, hydrocode simulation, hardware implementation, testing, and analysis leading to a final weapons system. While the nuclear weapon design iteration that leads to an experiment took up to five years and cost $\$ 5$ to $\$ 50$ million, a conventional weapon design iteration can be completed in a month or two at a cost of $\$ 50,000$ to $\$ 200,000$.

- Implosion Dynamics. The explosive in a nuclear weapon implodes a metal liner. The same is true in a military shaped charge, and some of the details for precision mass motion are even more demanding. There is significant interest in the latest generation of shaped-charge technology for anti-armor munitions, hard-target lethality for counterproliferation interdiction missions, mine clearing, cutting surplus metal from excess retired military hardware, and as a "jet $a x^{\prime \prime}$ for rapid access to fires and terrorist or hostage situations. The manufacture of low-cost, highprecision, special metal liners for these shaped charges is a challenge that industry has embraced.

- Codes, Simulation, and Modeling. The numerical simulation that is needed for nuclear warhead design and safety assessments is the same as that needed for conventional (nonnuclear) warhead design and for target interaction, lethality, and vulnerability (TILV) assessments. The DoD has applied codes developed for nuclear weapons in new regimes, which has led to the development of improved 
zoning techniques, improved algorithms, and enhanced material models and has hastened the development of $3 \mathrm{D}$ codes. These, in turn, have made possible evaluations of nuclear weapons safety in a variety of threat scenarios. Continuing challenges are driving the development of more efficient codes, such as 3D

Eulerian or arbitrary Lagrangian-Eulerian (3D ALE) codes and massively parallel processing (MPP) for nuclear weapons design and safety studies and for conventional weapons design and TILV assessments. Traditionally, the DoD has relied on the hydro-dyanmics codes developed by the weapons laboratories for its needs.

The defense industry uses these codes for modeling stealth properties, weapons design, and TILV. The civilian industry relies on codes such as DYNA3D, NIKE3D, MESA, CTH, and others for modeling the impact dynarnics of human bodies for improved crashworthiness, structural damage of aircraft by either impacting birds or fractured turbine blades, electromagnetic effects on fly-bywire aircraft, electromagnetic interference in tightly packed IC designs, and electronic packaging.

The weapons laboratories have also developed conflictsimulation codes for tradeoff studies to assess tactical nuclear vs conventional weapons in a battlefield environment. These codes are now used by the military to train battlefield commanders, assess the value of nonnuclear weapon systems, and conduct mission rehearsals (e.g., for Operations Just Cause and Desert Storm) and site-security exercises.

\section{- Diagnostics, Data Manage-} ment, and Communications. The need to benchmark new shaped-charge implosion calculations has led to the development of a stop-action (20-ns), laser-illuminated, electro-optic camera that provides sequential stopaction photographs of a shaped charge during jet formation and interaction with a target. This and related technologies have added to assessments of the implosion dynamics of nuclear weapons using surrogate materials. In addition, improved data acquisition from nonnuclear testing has been aided by significant developments in broadband fiber optics technology. This same technology is the backbone of the communications sector. Neuralnetwork chip technology for signal processing can be used for smart fuses in conventional weapons, data compression for transmitting large quantities of data, automatic decision-making, and the detection of sea-skimming missiles attacking ships.

- Advanced Materials. Materials originally developed for nuclear weapons are used in a spectrum of military applications.

* Fiber composites are used in aerospace and aircraft applications and in lightweight launchers, sabots, and highcapacity artillery shells. The DOE contribution to this effort is the 3D design and use of fiber composites to near their theoretical mechanics limits.
* Aerogels are lightweight, highly porous substances that can be used as aerocapacitors.

* Cermets are metal-infused ceramics that have military applications in armor.

* Nanostructures are materials that are constructed one atomic layer at a time and have alternate layers of materials. Nanostructures have produced the hardest, most fracture-tough materials known for use as coatings in aircraft engines. They are also used in the development of high-energy capacitors for military electromagnetic/electrothermal guns. Additional material developments include diamond and diamond-like coatings for wearresistance and thermal management applications and strainlayer compound semiconductor materials for opto-electronic and high-frequency electronics devices. - Nondestructive Testing. Techniques developed to inspect nuclear weapons components have found a wide application in the military sector. They can be used to study the combustion stability and dispersal of reacted materials in a rocket engine undergoing testing, for assessing the service life of military and civilian aircraft, for determining the tritium levels in nuclear configurations, and for finding unexploded ordnance (e.g., mines) and buried waste sites.

- Environmental Technologies. Environmental technologies developed for such nuclear weapons issues as treaty verification, nonproliferation, and the cleanup and restoration of the nuclear weapons complex have 
multiple applications. Examples include plasma torches for the benign incineration of waste materials as well as molten salt destruction, moderated electrochemical oxidation, and supercritical water oxidation for the demilitarization of high explosives and other materials and parts from dismantled weapons. - Explosives and Initiation Systems. The DoD uses insensitive high explosives developed by the DOE for nuclear weapons in weapons such as the TOW and Hellfire missiles. Slapperbased initiation systems, which were invented and developed for nuclear weapons, are used in the fuses of many DoD weapons. In addition, insensitive high explosives that were invented and developed by the weapons laboratories are being studied for possible use in some special-purpose conventional munitions.

- Electromagnetics. Electromagnetic codes developed to study the effects of nuclear weapons systems are being used to determine the susceptibility of airplanes to radiofrequency pulses from radar and other phenomena. They are also being applied to military interests in high-power microwave and ultrawide-bandwidth radar for intrusion detection, object identification, and attacking missile guidance and control disruption.

- Laser Technology. Lasers have played an important role in the design and fabrication of nuclear weapons. Lasers and laser technologies were developed initially for inertial confinement fusion (ICF) and to support other high-energy-density applications. Today, lasers are used to simulate nuclear explosions, for isotope separation, and for a variety of diagnostics including linear, nonlinear, and kinetic spectroscopy. The military applications of lasers include mine detection, antisubmarine warfare, directed-energy weapons, antisensor weapons, strategic laser communications, ultra wide-bandwidth radar and communications, high-resolution imaging, remote sensing, tactical battlefield systems, strategic defense weapons, chemical and biological warfare (CBW) remote detection, CBW material destruction (remote/ local), and weapons safety and optical fusing. A lidar system was deployed in Operation Desert Storm to provide advanced detection of chemical and biological weapon hazards.

\section{Fundamental Science}

Science-based stockpile stewardship must be built on a broad scientific and technical base. To develop the necessary fundamental and predictive capability, we must engage in a wide variety of scientific and technical activities and develop powerful experimental and computational capabilities. We must understand everything from the physical processes in a nuclear weapons explosion to the subtheties of materials aging. The results of what we learn will be of interest to the broader scientific community.
The DOE facilities and capabilities are significant scientific tools. Used effectively, they can encourage scientists to work with weapons personnel on problems related directly to stockpile stewardship as well as to issues in the same or related disciplines.

- Astrophysics and Space Physics. The unusual highenergy-density plasmas that occur in nuclear weapons are closely related to the dense, hot plasmas that make up many astronomical objects in our solar system, in other stars, or in other galaxies. The atmospheres of the gas-giant planets (Uranus and Neptune) are at ultrahigh pressures and densities that affect their atomic and physical properties. Astrophysicists attempt to decipher the few clues provided by Earth-based telescopes and space probes like Galileo to learn about an object's nature. Most often, these clues consist of plasma radiation or absorption spectra, requiring that the physicist understand atomic physics in dense plasmas and radiation interactions with plasma.

Experiments with stockpile stewardship facilities (principally lasers, but including pulsedpower facilities) can create and probe astrophysically relevant plasmas for studies of their properties. Important properties include the radiative opacities of the outer envelopes of stars, which are essential to our understanding of how stars evolve; the equations of state of stellar material, which are a major determinant of how 
dwarf stars behave; and mixing of materials at hydrodynamically unstable material interfaces, which is particularly important in understanding supernovas. Pulsed-power facilities can compress centimeters of material to megabar pressures for a microsecond, making it possible to create and study the properties of planetary-atmospherelike samples of hydrogen, helium, and appropriate trace elements. Radiation opacity work with the Nova laser has already provided valuable information for the astrophysics community.

The analysis and modeling of complex systems involving hydrodynamics, radiation transport, and nuclear burning play a central role both in astrophysics and in our understanding of nuclear weapons and their effects. Examples include supernovas, stellar evolution, cosmology, nucleosynthesis, gamma-ray bursts, and binary $x$-ray sources. The physics understanding and computational techniques developed in the weapons program have helped us understand these phenomena, while techniques (and trained personnel) in the astrophysics community have made a major contribution to the weapons program. The continued vigorous development of the improved predictive capabilities needed to ensure the safety and security of the nuclear stockpile will further enhance this synergy. Observation, analysis, and modeling of explosive astrophysical events will offer a continuing and increasingly sophisticated means of testing our understanding of the complex interactions of various physical processes in nuclear weapons. In this way, astronomy will provide another laboratory and an additional dataset. A merger of weapons-related and astrophysical data will improve our knowledge of the universe and our understanding of nuclear weapons.

- Hydrodynamics. The detailed understanding of hydrodynamics and fluid dynamics that is fundamental to the Stockpile Stewardship Program will also benefit fundamental science and computational modeling. Stockpile stewardship requires that we understand shock interactions, instabilities, shear, turbulence, and other nonlinear hydrodynamic phenomena in complex geometries and over a broad range of conditions (from low-pressure shocks, where the strength of materials may be important, to the regime of dense plasmas). Hydrotest facilities, explosively driven experiments, and pulsed-power hydrodynamic experiments are providing increasingly detailed data about these complex phenomena. Our physics and hydrodynamics models must be validated against these (and future) data.

Improvements in the fundamental understanding of hydrodynamics will benefit such diverse applications as conventional (nonnuclear) defense and internal combustion engines.

High explosives, pulsed power, or lasers can be used to deposit large amounts of energy at high power, generating very- high-density, high-temperature volumes of material that can then be used to drive strong shocks and high-velocity flows. An extensive set of diagnostics has been developed to measure interfacial geometries, velocities, densities, temperatures, and other relevant properties. The stockpile stewardship facilities provide for larger scales, longer times, and higher energies than are available at most universities or industries. These facilities will permit experiments to be larger (which increases the relative resolution), longer (which allows for greater development of features that change with time), and more energetic (which helps maintain the drive conditions and reduce compression effects). Such experiments will improve our understanding of fluid dynamics and will serve as test problems for the new generation of fluid-dynamics computer simulations. They will also make it possible to investigate such topics as hydrodynamic instabilities in planar and convergent geometries, compressible turbulent jets, shear layers, turbulent mixing, shock hydrodynamics (including shock-shock interactions and analogies to nonequilibrium gas dynamics), hypervelocity flyer plates, and possibly some magnetohydrodynamics (MHD) problems.

\section{- Condensed Matter.}

Condensed matter science, particularly materials science, will be of increasing importance in science-based stewardship largely because of issues related to aging and its effects: 
* Effects of helium accumulation (due to tritium decay) in plutonium.

* Aging of materials, including aging in the presence of low-level radiation.

* Detailed processes of interfacial chemistry and corrosion.

* Structure and its role in the properties of explosives.

* Control of material properties for component manufacturing.

Condensed matter science will also be important for advanced manufacturing ranging from plutonium components to microelectronics.

Stockpile stewardship facilities such as LANSCE (a pulsed source of neutrons with a broad range of energies) are beneficial for both condensed matter and nuclear science and can be used for applications relating to plutonium aging, materials issues, and the nondestructive evaluation of weapon subassemblies and components.

Neutron scattering, for example, plays an important role in understanding the fundamentals of magnetism. It also contributes to improving magnetic recording technologies with studies of the magnetic structure in fine-grained materials. Neutron scattering plays a role in understanding and improving models of strain in the metal-matrix composites used as lightweight, high-strength industrial materials and in understanding how hydrogen behaves in metals and causes changes in materials properties. At the molecular level, neutron scattering has been used to explain the 3D structure and bonding of complex molecules associated with chemical catalysis (such as metal dihydrogen complexes) and to probe the structure of high-temperature superconductors. Often, we can make complete determinations of such structures by comparing complementary $x$-ray and neutronscattering data.

Neutron scattering is an integral part of a revolution in structural biology, providing probes of the structure of proteins and viruses. The 3D structure of such bio-molecules is often a key to their function. LANSCE has been used to determine the interaction of calmodulin, a protein that mediates the regulatory effect of cal-cium in many biological processes with enzymes. This facility has also been used to produce a number of important radioisotopes for medicine.

Bioscience benefits from other unique capabilities developed initially for stockpile stewardship. The computational capabilities of the weapons laboratories, in particular, have made significant contributions to the Human Genome project, an international endeavor to completely map the 23 pairs of human chromosomes, and to the HIV database that compiles everything known about the gene sequences of the virus that causes AIDS. In addition, researchers using Nova and other high-power lasers are developing $x$-ray lasers of the proper wavelength to provide high-contrast, molecular-scaleresolution holograms of cells and other biological structures.

Ultrahigh (megagauss and more) magnetic fields are another condensed-matter probe that benefits stockpile stewardship as well as the pulsed-power and materials communities. Some of the pulsed-power capabilities developed for stockpile stewardship provide otherwise impossible measurements. For example, measurements to $3 \mathrm{MG}$ of the critical field of the high-temperature superconductor YBCO (the critical field is the field at which superconductivity is quenched) were recently made by a U.S.-Russian team in a collaborative effort for the scientific conversion of activities at Russia's weapons laboratory Arzamas-16. A number of other applications are being explored with the National High Magnetic Field Laboratory. Condensed matter physics at extreme pressures and temperatures, at high strains and high strain rates near melt or ionization, and at even more extreme conditions of energy density will also be a critical activity in stockpile stewardship. There are many uncertainties about the detailed transient behavior of materials under these conditions. Differing approaches can be taken to the microscopic materials models that describe plastic flow, fracture, and melt phenomena in the lower-pressure, high-strainrate regime where materials strength and the memory of 
initial materials structure can be important. These models can predict very different behavior, and there are few detailed data to distinguish them. No models of the material equation of state for pressures between 1 and 100 TPa have been validated experimentally, although considerable equation-of-state data exist at pressures below a few megabars. At very high pressures, statistical-mechanical model theories are generally thought to be valid. At tens of terapascals (hundreds of megabars), existing models agree only approximately in density and may be worse in temperature along a shock adiabat or Hugoniot. Although a few shock-wave experiments (driven by a nuclear device) in this pressure range have been conducted in Russia, none has been sufficiently accurate to distinguish among the various theories.

Nanometer-size clusters of matter (consisting of tens to a few thousand atoms) and assemblies made from them represent a new class of materials with unusual properties. These properties derive from the unconventional physical and chemical properties of the clusters and the strong dependence of those properties on cluster size. The physics and chemistry that control the behavior of nanoclusters differ greatly from those of individual atoms or bulk solids for a number of reasons, including:

* The large fraction of constituent atoms at or near the surface.
* The unique bonding configuration and geometrical structures.

* The quantum confinement of electronic wavefunctions that modify the energy-level structure. Nanocluster research has been hampered by the lack of appropriate synthesis techniques that can produce useful amounts of size-selected, surface-stabilized clusters. Recently, pioneering research, supported by stockpile stewardship activities, overcame this limit with a method based on the use of well-defined, nanometer-size interiors of inverse micellar cages as reaction vessels for the growth of size-selected metal, semiconductor, and oxide clusters. Size selection allows the properties of materials to be tuned, leading to novel applications for sensors, pollutionemission control, and environmental restoration. New classes of tailored bulk materials built by assembling individual clusters are now being studied; they include homo- and heterogeneously assembled ceramics, metals, and semiconductors for novel mechanical, electronic, and optical devices.

Understanding and predicting the effects of aging on the components and materials used in nuclear weapons (stockpile stewardship requirements) require techniques that can probe surface and interface properties at the atomic level to study problems like adhesion failure, interdiffusion, and corrosion. The interfacial force microscope, developed with support from the DOE Office of Defense Programs, now makes it possible to study interfacial adhesion at the atomic level. Scientists involved in such research have theoretically predicted and experimentally verified a new mechanism for surface diffusion and interfacial mixing, pointing the way to the development of a predictive capability for interfacial stability. They have also developed a broad understanding of how surface chemistry and mechanical properties relate to the stress-corrosion degradation of materials.

\section{- Microelectronics and}

Photonics. Research in photonic lattices (pioneered by the weapons program) holds promise for a new class of photonic and electronic devices. New or improved semiconductor materials often pace the development of higher-performance microelectronic devices and microsensors crucial to stockpile stewardship. Research supported by the weapons program led to the development of strained-layer superlattice materials that now serve as the substrates for vertical-cavity semiconductor laser devices and ultrahigh-speed electronic devices.

Recent pioneering research to control the propagation of light to specific wavelengths and angles using $2 \mathrm{D}$ lateral patterning of electronic and photonic materials on nanometer-length scales is opening a new field of study that promises an extremely efficient, low-power, zero-threshold laser. Similarly, the coupling of large arrays of semiconductor lasers into specially designed photonic lattice structures may 
enable ultralow-divergence, highpower, directionally controlled laser sources.

These new materials control the photons in a manner that is analogous to the way that the periodic arrangement of atoms in semiconductor materials determines electron propagation. Lateral and vertical patterning of semiconductor materials at length scales comparable to the electron wavelength or phasecoherence length have led to a new physics understanding of the quantum-wave interference of electrons (analogous to the interference of light waves). The sharp tunneling resonance that results from this phenomenon holds promise for a new class of extremely fast, low-power electronic devices.

- Vision Science. Vision-science activities in the weapons program are motivated by a need to develop machine vision for robotics and for sensor-controlled manufacturing. At present, many human visual-perception processes cannot be duplicated by computer. However, a recent weapons research project achieved a breakthrough in understanding how the human visual system perceives and computes clusters of pattern vectors in a dataset or image. This understanding, in turn, has led to the development of a pattern-recognition technique that closely mimics human visual performance.

- Plasma and Atomic Physics. The study of plasma physics has been motivated by the desire for an energy source that has a virtually inexhaustible supply of raw material. The further realization that the universe is made primarily of plasma makes the scientific investigation of plasma physics even more germane to scientific inquiry.

Plasma physics is also crucial to nuclear weapons. Some of the phenomena that occur in plasmas, such as parametric instabilities, rely on a large degree of homogeneity in hot, dense plasmas. However, most plasma-physics experiments to date have been complicated by the large gradients and small scales of the plasmas produced by current lasers. The advanced capability required for the Stockpile Stewardship Program will allow us to produce hot, dense plasmas that are both large and homo-geneous, permitting detailed characterization of their properties, including electron and ion temperatures, charge states, electron density, and plasma-flow velocities.

The short-pulse, high-power experiments needed for stockpile stewardship open the door to many basic plasma-physics studies, including:

* Relativistic, ultrahighintensity regimes of laser-matter interaction.

* Ponderomotive effects.

* Relativistic self-focusing and filamentation.

* Laser-beam channeling.

* Intense harmonic generation.

* Ultrahigh magnetic-field generation with ancillary studies of the resulting physical processes. regimes.

* Strongly driven instability

* Generation and transport of high-electron fluxes in plasmas. schemes.

* High-gradient accelerator

* X-ray lasers.

Another new area of focus will be the study of the strong magnetic fields (sometimes as large as $100 \mathrm{MG}$ ) that are generated by temperature and density gradients in plasmas. Zeeman splitting of spectral lines should allow researchers to investigate and quantify these magnetic fields. The generation of large currents of fast electrons coupled to a high- $Z$ (atomic number) metal converter should allow the production of short-duration bursts of $50-$ to $100-\mathrm{kV} x$ rays with numerous potential radiographic applications. It should also be feasible to perform nuclear-reaction experiments in the large, hot, well-characterized plasmas.

- The larger plasmas possible at new Stockpile Stewardship Program facilities will allow longer interaction times and greater homogeneity, meaning that the spatial resolution requirements on temperature and density characterization would be relaxed. The higher temperatures and longer time scales will also allow a relaxation of ion-electron temperatures. In this way, the Stockpile Stewardship Program facilities will make strong contributions to fundamental plasma physics and some more esoteric studies.

$X$-ray lasers are important coherent radiation sources and they provide critical tests of our atomic modeling capabilities. The production of $x$-ray lasers using new stockpile stewardship facilities will allow us to extrapolate 
Appendix

from existing neon- and nickellike collisional $x$-ray lasers to lasers with wavelengths of about $200 \mathrm{~nm}$. At these wavelengths, $x-$ ray-laser interferometry can be used to measure electron densities in plasmas exceeding solid densities. Short-pulse laser capabilities will allow us to develop inner-shell, pumped $x$-ray lasers as viable radiation sources. This new class of $x$-ray laser has the potential to extend down to 10 $\mathrm{nm}$ in wavelength with a short pulse width. High-order harmonic generation, an alternative source of coherent radiation, can be used to produce tunable extreme-ultraviolet coherent radiation.

- Fusion. Some of the science required for stockpile stewardship-for example, that related to the atomic physics of dense plasmas-may have indirect benefits to aspects of magnetic fusion. However, the primary connection of stockpile stewardship to fusion energy is through the explicitly dual-benefit national Inertial Confinement Fusion (ICF) program. The ICF program, funded through DOE's Office of Defense Programs, seeks as its next step to demonstrate thermonuclear ignition and modest energy gain in the National Ignition Facility (NIF). The significant progress in ICF over the last two decades, achieved in large part through the use of classified capabilities and expertise of the weapons laboratories, has made ICF credible as an approach to fusion energy. Demonstration of ignition and energy gain with the NIF (the principal design goal of that facility) would provide a major impetus for explicit development of ICF for energy applications. The recent declassification of significant portions of ICF science will lead to a broader involvement of the U.S. and international scientific communities in the development of this approach to fusion energy. - Nuclear Science. Historically, the nuclear weapons program underw rote significant elements of nuclear physics research in order to obtain the accurate nuclear reaction data needed for both weapon performance and weapon diagnostics. Nuclear science continues to be important for stewardship of the stockpile and for reducing global nuclear danger, with measurements of reaction cross sections and the development of accelerator methods for producing tritium for the stockpile and for transmuting hazardous wastes and plutonium.

The challenges of sciencebased stockpile stewardship will provide important opportunities for advances in basic and applied nuclear science. LANSCE, with its unique capabilities for neutronbased nuclear science, will be the major contributor to this effort. Although used principally for stockpile stewardship, LANSCE is also a unique facility for experiments that are of interest to the nuclear physics community (for example, sensitive studies of parity violation and neutrino physics). - Radiation Sources. The Stockpile Stewardship Program facilities, including Atlas, Jupiter, and the National Ignition Facility, will provide a wide variety of $x$-ray and particle sources suitable for addressing basic and applied research areas. These facilities will be able to produce intense broadband thermal $x$ rays, coherent amplified $x$ rays ( $x$-ray lasers) from high-gain linear plasmas, intense neutron pulses from implosion plasmas, and intense pulses of hard $x$ rays produced by fast electrons. Broadband $x$ rays generated from high-temperature, highdensity plasmas driven by lasers or pulsed-power sources can be used to produce and characterize large, uniform plasmas that are relevant to fusion and astrophysics. Previous experiments have yielded results that can be extended to high temperatures and densities.

- Environmental Monitoring and Science. Stockpile stewardship capabilities and skills can contribute to environmental monitoring and technologies in a number of areas, such as remotesensing lidar. In 1993, a team from the nuclear weapons test program successfully fielded a shipboard lidar as part of the Central Equatorial Pacific Experiment (CEPEX) to provide data for climate models.

Computational capabilities, such as massively parallel processing and three-dimensional modeling of complex hydrodynamics, offer valuable opportunities to contribute to global climate modeling. The weapons laboratories are collaborating with academia and other research centers to improve our predictive modeling capabilities; a case in point is a recent breakthrough in global ocean modeling. 
Technologies developed in the Stockpile Stewardship Program for reducing the amount of waste and detrimental environmental effects resulting from the manufacturing and processing of weapons components benefit the environment and U.S. industry. For example, cleaning technologies that use nonchlorofluorocarbon solvents, methods for environmentally benign disposition of excess explosives, and waste minimization technologies in machining have been developed, in many cases in direct collaboration with industry. The technology base for an accelerator for producing tritium (a more environmentally benign method than current processes) is closely related to that for the accelerator transmutation of long-lived radioactive waste (a process with potential for solving the problem of long-term storage of radioactive waste).

- Weapons Systenns Tests and Demonstrations: The Nevada Test Site, with its vast remote area, its facilities for testing high explosives and nuclear material, and its proximity to numerous military facilities, has supported a variety of military experiments in the past. Live firing of munitions containing depleted uranium is one example. The Nevada Test Site is an ideal test range for tests or demonstrations involving weapons systems and nuclear materials (actual or simulated) to develop technologies for preventing or countering the proliferation of nuclear weapons.

- High Explosives and Nuclear Materials Tests: Although some experiments with high explosives and nuclear materials can be done in a laboratory environment, the Nevada Test Site provides the added margin of safety required for large-scale experiments. Complex experimental configurations and large amounts of energetic or hazardous material can be tested in tunnels or other underground facilities with minimal safety or environmental risk.

- Large-Scale Chemical Tests: A national spill test facility has been in operation at the Nevada Test Site for many years.

Originally developed to perform safety-related tests in support of the liquefied gaseous fuels industry, it has been expanded to become both a sensor test range and a general spill test experimental facility. Its predictable atmospheric conditions, long downwind controlled area, and existing test infrastructure make it the best large-scale spill test facility in the nation.

- Conventional Munitions Tests and Demonstrations: The Nevada Test Site has been used for large-scale safety assessments of munitions and propellants in abnormal environments (e.g., will a rocket motor burn or explode if engulfed in a fire?). With the general builddown of the U.S. military in the post-Cold War environment, there are new needs to address the destruction, disposal, and recycling of munitions and rocket motors. In collaboration with the nearby demilitarization research and development facilities at the Hawthorne Depot, the Nevada Test Site is engaged in experiments to burn or otherwise dispose of rocket motor propellants. In collaboration with the remote sensing capabilities of the DOE weapons laboratories, the DoD is also using the Nevada Test Site as a test range for mine detection. 


\section{Acronyms}

\begin{tabular}{|c|c|c|c|}
\hline AFF & arming, fuzing, and firing & FXR & Flash X-Ray facility (Livermore) \\
\hline AGX & aboveground experiments & & \\
\hline AHF & $\begin{array}{l}\text { Advanced Hydrotest Facility } \\
\text { (proposed) }\end{array}$ & giga & $10^{9}$ \\
\hline ALE & arbitrary Lagrangian-Eulerian & $\begin{array}{l}\text { HE } \\
\text { HN }\end{array}$ & $\begin{array}{l}\text { high explosive } \\
\text { hydronuclear }\end{array}$ \\
\hline$C^{3} I$ & command, control, communica- & & \\
\hline $\mathrm{CAD} / \mathrm{CAM}$ & $\begin{array}{l}\text { tions, and intelligence } \\
\text { computer-aided design/computer- } \\
\text { aided manufacturing }\end{array}$ & IAEA & $\begin{array}{l}\text { International Atomic Energy } \\
\text { Agency } \\
\text { integrated circuit }\end{array}$ \\
\hline CBW & chemical and biological warfare & ICBM & intercontinental ballistic missile \\
\hline CD & command disable & ICF & inertial confinement fusion \\
\hline CD & Conference on Disarmament & IHE & insensitive high explosive \\
\hline CEPEX & $\begin{array}{l}\text { Central Equatorial Pacific } \\
\text { Experiment }\end{array}$ & IMTL & $\begin{array}{l}\text { Integrated Manufacturing } \\
\text { Technology Laboratory (Sandia) }\end{array}$ \\
\hline CFC & chlorofluorocarbon & INS & Immigration and Naturalization \\
\hline CMR & $\begin{array}{l}\text { Chemistry and Metallurgial } \\
\text { Research laboratory (Los } \\
\text { Alamos) }\end{array}$ & $\begin{array}{l}\text { I/O } \\
\text { IR }\end{array}$ & $\begin{array}{l}\text { Service } \\
\text { input/output } \\
\text { infrared }\end{array}$ \\
\hline CRADA & $\begin{array}{l}\text { cooperative research and develop- } \\
\text { ment agreement }\end{array}$ & JRMB & Joint Requirements and \\
\hline $\begin{array}{l}\text { CTB } \\
\text { CWC }\end{array}$ & $\begin{array}{l}\text { comprehensive test ban } \\
\text { Chemical Warfare Convention }\end{array}$ & & Management Board (DoD) \\
\hline & Cinenucal vadiale Coitvenitionil & LANSCE & $\begin{array}{l}\text { Los Alamos Neutron Scattering } \\
\text { Center }\end{array}$ \\
\hline 20,30 & $\begin{array}{l}\text { two dimensional, three dimen- } \\
\text { sional }\end{array}$ & laser & light amplification by stimulated \\
\hline DARHT & $\begin{array}{l}\text { Dual Axis Radiographic Hydro } \\
\text { Test facility (Los Alamos) }\end{array}$ & & emission of radiation \\
\hline DoD & Department of Defense & lidar & $\begin{array}{l}\text { limited-lifetime component } \\
\text { laser infrared radar }\end{array}$ \\
\hline DOE & Department of Energy & & \\
\hline DP & Defense Programs (DOE) & MDL & $\begin{array}{l}\text { Microelectronics Development } \\
\text { Laboratory (Sandia) }\end{array}$ \\
\hline EBГT & $\begin{array}{l}\text { Electron Beam Ion Trap } \\
\text { (Livermore) }\end{array}$ & $\begin{array}{l}\text { MHD } \\
\text { MPP }\end{array}$ & $\begin{array}{l}\text { magnetohydrodynamics } \\
\text { massively parallel processing/ }\end{array}$ \\
\hline EMP & electromagnetic pulse & & processor \\
\hline $\begin{array}{l}\text { ENDS } \\
\text { EOS }\end{array}$ & $\begin{array}{l}\text { enhanced nuclear detonation safety } \\
\text { equation of state }\end{array}$ & MRI & magnetic resonance imaging \\
\hline ER & Energy Research (DOE) & NEST & Nuclear Emergency Search Team \\
\hline ESD & environmental sensing device & NIF & National Ignition Facility (proposed) \\
\hline ES\&H & environment, safety, and health & $\begin{array}{l}\text { NPT } \\
\text { NSL }\end{array}$ & $\begin{array}{l}\text { Non-Proliferation Treaty } \\
\text { National Storage Laboratory }\end{array}$ \\
\hline FEMA & $\begin{array}{l}\text { Federal Emergency Management } \\
\text { Agency }\end{array}$ & NTS & Nevada Test Site \\
\hline flop(s) & $\begin{array}{l}\text { floating-point operation (per } \\
\text { second) }\end{array}$ & $\begin{array}{l}\text { PAL } \\
\text { peta }\end{array}$ & $\begin{array}{l}\text { permissive action link } \\
10^{15}\end{array}$ \\
\hline $\begin{array}{l}\text { FRP } \\
\text { FSU }\end{array}$ & $\begin{array}{l}\text { fire-resistant pit } \\
\text { former Soviet Union }\end{array}$ & PETL & $\begin{array}{l}\text { Processing and Environmental } \\
\text { Testing Laboratory (Sandia) }\end{array}$ \\
\hline
\end{tabular}


PHERMEX Pulsed High-Energy Radiography

radar RADFET rf RMSEL

SNM START

TA-55

Technical Area 55 (Los Alamos Machine Emitting X Rays (Los Alamos)

radio detection and ranging radiation field-effect transistor radiofrequency

Robotic Manufacturing Science and Technology Laboratory (Sandia)

special nuclear material Strategic Arms Reduction Talks/Treaty

tera

TIVL

TOW

TPP

YBCO

Z
$10^{12}$

target interaction, lethality, and vulnerability

tube-launched, optically tracked, wire-guided missile

Technology Partnership Program (DOE)

yttrium barium copper oxide $\left(\mathrm{YBa}_{2} \mathrm{Cu}_{3} \mathrm{O}_{7}\right)$, a hightemperature superconductor

atomic number plutonium facility) 
adiabatic

aerogel

agile manufacturing

algorithm

arbitrary LagrangianEulerian

bandwidth

benchmark

boost

burn

carrier

cermet
Referring to any change (e.g., compression, expansion, passage of a shock wave) in which there is no gain or loss of heat.

A class of extremely lightweight, porous materials composed of interconnected clusters of atoms; the extremely high surface area produces a nanostructure with unique optical, thermal, acoustic, mechanical, and electrical properties.

Effective use of equipment and people, involving time-sharing of expensive equipment and broader responsibilities for workers on the manufacturing floor, to rapidly and cost-effectively meet the needs of high-quality, lowvolume production.

A procedure or set of rules, commands, or instructions, usually but not necessarily given to a computer, for solving a mathematical problem or performing a task in a finite number of steps and usually involving repetition of an operation.

A zoning technique that permits the use of an embedded Lagrangian mesh, a fixed Eulerian mesh, or a partially embedded, partially remapped mesh. The problem is usually run so that the mesh starts out completely Lagrangian. Remapping begins after significant mesh distortion is detected; remapping is confined to the distorted region. Remapping across material boundaries is permitted. A switch to an Eulerian mesh can be made as the program progresses. Expert knowledge is usually required to make the decisions needed to set up this type of program.

The range within a band of wavelengths, energies, or frequencies containing the useful components of a signal.

A point of reference from which measurements can be made; something that serves as a standard by which others can be measured.

The process by which fusion of deuterium-tritium gas inside the pit produces neutrons that increase the fission output of the primary.

Fusion of two light nuclei (usually deuterium and tritium) to form a heavier nucleus (helium) accompanied by the release of neutrons and energy.

The military "vehicle" (e.g., ballistic or cruise missile, artillery shell, airplane, submarine) by which a nuclear weapon would be delivered; most warheads have been designed for specific carriers (analogous to "delivery system").

A class of ceramic-metal composite materials remarkable for their light weight and high strength. 
coherent radiation

command disable

common-mode failure

computational modeling

concurrent engineering

conventional weapon

Coyote Test Range

deflagration

delivery system

detonation

dimensionality

dual use/dual benefit

electrical nuclear

detonation safety
Radiation in which there are definite phase relationships between two or more beams or between different points in a cross section of a beam, so that interference effects can be produced between them.

A subsystem of command and control features that destroy a weapon's ability to produce nuclear yield.

A failure or defect affecting an entire class of weapon or weapon component; a particular concern with the enduring stockpile since it contains fewer than ten weapon systems, many of which use identical components, components with common design features, or components manufactured using identical or similar processes.

The use of a computer to develop a mathematical model of a complex system or process and to provide conditions for testing it.

Concurrent design of both the product and the processes for manufacturing the product; integrated design, production, prototyping, and product qualification.

A nonnuclear weapon.

Test range, located at Kirtland Air Force Base (Albuquerque, NM) and operated by Sandia, with a wide array of weapon environmental test facilities.

Rapid and powerful self-sustained burning of a propellant or explosive.

The military "vehicle" (e.g., ballistic or cruise missile, artillery shell, airplane, submarine) by which a nuclear weapon would be delivered; most warheads have been designed for specific delivery systems (analogous to "carrier").

An exothermic chemical reaction that propagates with such rapidity that the rate of advance of the reaction zone into the unreacted material exceeds the velocity of sound in that material; that is, the advancing reaction zone is preceded by a shock wave.

The number of dimensions a computer simulation can model; most problems of concern to stockpile stewardship are three dimensional in character, but many of our codes are limited to two dimensions.

Projects that have uses in or benefits for the defense sector and the private industry or civilian sector.

The prevention of unintentional electrical detonation of a nuclear weapon, achieved primarily by incorporating electrical exclusion regions that protect the electrical firing system from unintended sources of electrical energy. 
empirical

enduring stockpile

energy

energy density

energetic material

enhanced nucleair

detonation safety

environmental sensing

device

Eulerian

explosion (conventional)

fiber composite

fire-resistant pit

floating-point operation
Something that is based on actual measurement, observation, or experience rather than on theory.

The U.S. nuclear stockpile of the future, consisting of fewer than ten weapon systems (many of them older than their design lifetime), with no new systems added to the stockpile for the foreseeable future.

The capacity for doing work.

The energy per unit volume of a medium (e.g., a plasma).

Generic term for high explosives and propellants.

The current standard for nuclear detonation safety, implemented through the use of sophisticated electrical firing system safety devices; specifies that the probability for unintentional nuclear detonation will be $<1$ in $10^{9}$ in normal environments and $<1$ in $10^{6}$ in accident or abnormal environments.

A safety device in the arming circuit of a weapon that prevents inadvertent function of the circuit until the weapon experiences an environmental change peculiar to its delivery method (e.g., pressure change, acceleration).

A zoning method for computational modeling in which the zoning mesh is fixed in space and the materials being modeled move through the stationary mesh. Since the mesh is fixed, mesh distortion does not occur; however, as the materials move through the mesh, some numerical diffusion is inevitably introduced, and, in a problem with several materials, mixed zones containing several materials can occur.

A chemical reaction or change of state that occurs in an exceedingly short time with the generation of high temperatures and large quantities of gaseous reaction products.

A composite material made of a continuous, chopped, or woven fiber (e.g., glass, graphite) in a matrix (e.g., resin, epoxy) formulated for specific applications to provide high strength and low weight, often with other accompanying characteristics (e.g., thermally insulating, electrically nonconducting, noncorrosive).

The fissile portion of a nuclear weapon (the pit), designed and constructed to resist the dispersal of plutonium and other fissile materials in the event of a jet-fuel fire or other high-temperature accident.

A mathematical operation carried out by a computer in which the decimal point is not fixed (i.e., allowed to float); usually involves numbers with exponents (e.g., 10 ${ }^{x}$ ). 
flops

\section{fluid dynamics}

fusion

high explosive

Hugoniot

hydrodynamics

hydrodynamic test

hydronuclear experiment

implosion

inertial confinement fusion

input/output (I/O)

insensitive high explosive
The number of floating-point operations per second that can be carried out by a computer; used as a measure of the processing capability of a computer.

The science of fluids in motion.

Nuclear reaction in which light nuclei are fused together to form a heavier nucleus, accompanied by the release of immense amounts of energy and fast neutrons.

An energetic material that detonates (instead of deflagrating or burning); the rate of advance of the reaction zone into the unreacted material exceeds the velocity of sound in the unreacted material.

A function specifying the locus of states that are possible immediately after the passage of a shock front; gives the state's pressure as a function of its specific volume.

The study of the motion of a fluid and of the interactions of the fluid with its boundaries, especially in the case of an incompressible inviscid fluid.

High-explosive nonnuclear experiment to investigate hydrodynamic aspects of primary function up to mid to late stages of pit implosion.

Very-low-yield experiment (less than a few pounds of nuclear energy released) to assess primary performance and safety with normal detonation.

The sudden inward compression and reduction in volume of a material; in a nuclear weapon, the fissionable material is imploded using high explosives.

The rapid implosion of a high-density pellet or target containing fusion fuel (usually deuterium and tritium) under bombardment of laser or chargedparticle beams; the target is heated almost instantaneously to extremely high temperatures to produce a core that undergoes fusion before the rest of the target flies apart.

Equipment and activity that transfers information into or out of a computer; the device that accepts new data, sends them to the computer for processing, receives the results, and translates them into a usable medium for the user.

A high explosive that is specifically formulated to be less sensitive to shock and other stimuli that might be encountered in an accident; usually based on the compound TATB (triaminotrinitrobenzene); insensitive high explosives have lower energy densities than conventional high explosives and thus more material is required to produce the same explosive energy. 


\section{Lagrangian}

limited-lifetime component

magnetohydrodynamics

massively parallel

processing

mesh refinement

microelectronics

microsensor

mix

mock nuclear material

nanostructure

nanocluster

nondestructive evaluation
A zoning method for computational modeling in which the zones move with the materials being modeled. Zones can be placed and will remain where they are needed most. This zoning technique can provide a very accurate representation so long as the mesh does not become extremely distorted, as can happen for very dynamic problems.

A weapon component that decays with age and must be periodically replaced.

The study of the dynamics or motion of an electrically conducting fluid, such as an ionized gas or liquid metal, interacting with a magnetic field.

An approach to high-performance computing in which many microprocessor-based computers (tens to hundreds) are interconnected, each with access to common data-storage volumes and other computing resources; the computation being run is broken into small segments and partitioned among the many processors, where each piece is solved in parallel with the others. This approach promises dramatic increases in computational speed.

The process of adjusting the computational mesh (see "Eulerian," "Lagrangian") during a numerical simulation to fix areas that have become tangled (a common occurrence when modeling dynamic processes affecting materials of different densities); until recently, this process had to be done by the person running the calculation, but routines are now available that enable the computer to identify and fix tangled regions of the mesh.

Integrated circuits and electronic devices constructed to fit in extremely small packages typically with dimensions of micrometers $\left(10^{-6} \mathrm{~m}\right)$.

A miniaturized sensing device; a type of microelectronics.

Mixing of materials, usually with different densities and velocities, that can adversely affect nuclear weapon performance.

Material that is not the actual fissile primary material but is similar in density and other characteristics to the actual fissile primary material and is used in place of a weapon's nuclear parts in hydrodynamic experiments and flight tests.

Miniaturized structure or device with dimensions of nanometers $\left(10^{-9} \mathrm{~m}\right)$.

Agglomerations or clusters of tens to thousands of atoms or molecules with dimensions of nanometers.

Test method that does not involve damage to or destruction of the test sample; includes the use of ultrasonics, radiography, magnetic flux, and other techniques. 
noninvasive imaging

nonnuclear component

nonproliferation

normalize

nuclear inheritor state

nuclear assembly

nuclear component

nuclear weapon

nuclear weapons complex

nuclear warhead

numerical simulation

opacity

performance

permissive action link

photonics
Imaging method that does not damage the test specimen; includes radiography, computed tomography, and other techniques.

Any one of thousands of parts that do not contain radioactive or fissile material that are required in a nuclear weapon.

Preventing the spread of nuclear weapons, nuclear weapon materials, and nuclear weapon technology.

To adjust the representation of a quantity so that the representation lies within a prescribed range; to adjust or calibrate a computer model so that its results fall within the normal range of empirical data.

The republics of the former Soviet Union that have nuclear weapons deployed on their territory; includes Russia, Ukraine, Belarus, and Kazakhstan.

Collective term for the primary, secondary, and radiation case.

A part of a nuclear weapon that contains fissionable or fusionable material.

The general name given to any weapon in which an explosion can result from the energy released by reactions involving atomic nuclei, either fission, fusion, or both.

The collection of laboratories, production plants, and military services involved in the design, production, and testing of nuclear weapons.

A warhead that contains fissile and perhaps fusionable material; the nuclear assembly and nonnuclear components packaged as a deliverable weapon.

The use of mathematical algorithms and models of physical processes to calculationally simulate the behavior or performance of a device or complex system.

The radiation flux incident upon a medium divided by the light flux transmitted by the medium.

The ability of a nuclear weapon or weapon system to operate in a specified manner (e.g., yield, range, accuracy, radiation spectrum) under stated conditions (essentially equivalent to "reliability").

A use control and security system that requires the receipt of a classified code to unlock a nuclear weapon in preparation for arming.

Scientific discipline dealing with the transmission of light (photons) and light-based devices; the light equivalent of electronics. 
plasma

power

product realization

reliability

retrofit

risk assessment

robust

safety

safety engineering

scientific visualization

security
An electrically neutral, gaseous mixture of positive and negative ions, sometimes called a fourth state of matter since it behaves differently from solids, liquids, and gases. High-temperature, high-density plasmas are created in nuclear weapons and ICF experiments.

The time rate of doing work or expending energy; measured in joules per second or watts.

The process that converts the nuclear assembly, nonnuclear components, subsystems, and system-level requirements into manufacturable designs and hardware.

The ability of a nuclear weapon, weapon system, or weapon component to perform its required function under stated conditions for a specified period of time (essentially equivalent to "performance").

To furnish (e.g., a weapon) with new parts, equipment, or features not incorporated at the time of manufacture.

A methodology for identifying potential risks, evaluating their probabilities of occurrrence, and weighting their severity relative to each other; used to formally assess the effectiveness of risk-reduction measures and to evaluate cost/value tradeoffs.

Sturdiness or ability of a weapon or component to withstand harsh environments; the sturdiness or lack of sensitivity of a computer simulation to modeling details.

Minimizing the possibility that a nuclear weapon will be exposed to accidents and preventing the possibility of nuclear yield or plutonium dispersal should there be an accident involving a nuclear weapon.

The aspect of system engineering that deals specifically with ensuring the safety of the nuclear weapon.

The transformation of numerical and symbolic information into readily interpreted and comprehensible visual formats; allows the scientist to gain greater insight into complex processes by providing a variety of ways of graphically representing quantitative information; employs a combination of computer graphics, image processing, numerical simulation, physics, chemistry, art, engineering, and other disciplines.

Minimizing the likelihood of unauthorized access to or loss of custody of a nuclear weapon or weapon system, and ensuring that the weapon can be recovered should unauthorized access or loss of custody occur. 
self-aware weapon

smart process

spatial resolution

special nuclear material

statistical mechanics

stockpile assurance

stockpile management

stockpile stewardship

stockpile surveillance

stockpile-to-target sequence
A stockpile weapon fitted with an integrated network of miniature "smart" sensors (sensing and measuring devices with built-in intelligence capabilities) and self-test features that monitor the weapon's environment (e.g., temperature, moisture, vibration), detect material decomposition products and corrosion, check cable continuity, determine the functionality of weapon subsystems, and alert a central location if any monitored parameters are outside the permitted range.

Manufacturing process that merges a computer-generated model of the product, electronic databases of materials properties, predictive computer models of the manufacturing process, and sensor-based adaptive control of the manufacturing process.

The fineness of detail about an object's structure or the space in which a process is occurring that can be handled by a computer model.

A specific list of materials including plutonium, uranium, and enriched uranium.

The branch of physics that endeavors to explain and predict the macroscopic properties and behavior of a system on the basis of the known characteristics and interactions of the microscopic constituents of the system (usually the number of such contituents is very large).

The umbrella term for stockpile management and stockpile stewardship; all the tasks required to ensure that the U.S. has a credible nuclear deterrent.

The specific tasks and functions involved in managing the stockpile weapons, including production, routine surveillance and servicing, assembly and dismantlement, and disposal of weapons-related parts and materials.

The science and technology aspects of ensuring the safety, security, and reliability of the stockpile, including research and development to provide the technologies required for stockpile management.

Routine and periodic examination, evaluation, and testing of stockpile weapons and weapon components to ensure that they conform to performance specifications and to identify and evaluate the effect of unexpected or age-related changes.

The range of environmental conditions, including temperature, moisture, acceleration, and vibration, which a weapon must be able to withstand and still function properly. 
strong-link switch

surety

superconductivity

superconductor

system integration

telemetry

test readiness

thermonuclear

tomography

Tonopah Test Range

use control

virtual corporation/ enterprise
Electromechanical device that prohibits the transmission of power and firing signals from being passed to the firing set except when it is activated by a unique electrical signal or a unique sequence of environmental stimuli.

Umbrella term for safety, security, and use control.

A property of many metals, alloys, and chemical compounds at temperatures near absolute zero in which their electrical resistivity vanishes and they become strongly diamagnetic.

Any material capable of exhibiting superconductivity.

Ensuring that the nuclear and nonnuclear components of a weapon meet performance requirements individually and as a system (see "weaponization").

Transmitting the readings of sensors and instruments to a remote location by means of wires, radio waves, or other transmission media.

Maintaining the critical technologies, staff skills, and infrastructure to be able to resume nuclear testing if and when mandated by the President.

The process by which very high temperatures are used to bring about the fusion of light nuclei, such as deuterium and tritium, with the accompanying release of energy.

Sectional radiography; a technique of making radiographs of plane sections of an object and combining the "slices" using sophisticated computer programs to produce a three-dimensional image of the object; a promising technique for noninvasive imaging and nondestructive evaluation.

Test range in Nevada, operated by the DOE, with unique capabilities for testing low-level, supersonic delivery techniques; the only U.S. location where weapon assemblies containing nuclear material can be tested.

Delaying or preventing the unauthorized use of a nuclear weapon or weapon system while allowing timely authorized use.

Integration of U.S. industry and the nuclear weapons complex to exploit each other's expertise, avoid duplication of facilities or staff, apply the most advanced product design and manufacturing processes, and provide ondemand manufacturing of weapon-related parts to maintain the enduring stockpile. 
virtual prototyping

warhead

weak-link switch

weapons laboratories

weapon system

weaponization
Complete simulation of the product design, performance, and manufacturing processes before any hardware is produced.

Collective term for the package of nuclear assembly and nonnuclear components that can be mated with a delivery vehicle or carrier to produce a deliverable nuclear weapon.

Device engineered to fail predictably and irreversibly when subjected to an abnormal environmental stimulus associated with accident phenomena (analogous to an electrical fuse).

Term for the three Department of Energy national laboratories-Los Alamos, Livermore, and Sandia-that are responsible for the design, development, and stewardship of U.S. nuclear weapons.

Collective term for the nuclear assembly and nonnuclear components, subsystems, and systems that comprise a nuclear weapon.

Converting the functional requirements for a weapon into integrated system designs and prototype hardware (see "system integration"). 